\title{
Copper-Catalyzed Enantioselective Ring-Opening of Cyclic Diaryliodoniums and $O$-Alkylhydroxylamines
}

\author{
Qigang Li, Mingkai Zhang, Shuming Zhan and Zhenhua Gu* \\ Department of Chemistry, Center for Excellence in Molecular Synthesis, and Hefei National \\ Laboratory for Physical Sciences at the Microscale, University of Science and Technology of China, 96 \\ Jinzhai Road, Hefei, Anhui 230026, P.R. China
}

zhgu@ustc.edu.cn

\section{General Information}

Nuclear magnetic resonances were recorded on Bruker-400 MHz instruments. Reference values for residual solvents were taken as $\delta=7.26 \mathrm{ppm}\left(\mathrm{CDCl}_{3}\right), 2.50 \mathrm{ppm}\left(\mathrm{DMSO}-\mathrm{d}_{6}\right)$ for ${ }^{1} \mathrm{H} \mathrm{NMR} ; \delta=77.00$ ppm $\left(\mathrm{CDCl}_{3}\right), 39.52 \mathrm{ppm}\left(\mathrm{DMSO}-\mathrm{d}_{6}\right)$ for ${ }^{13} \mathrm{C}$ NMR. High resolution mass spectral analysis (HRMS) was performed on Waters XEVO G2 Q-TOF (Waters Corporation). All reactions were performed under an inert atmosphere of dry nitrogen in flame-dried glassware, unless otherwise stated. Tetrahydrofuran were distilled over sodium in the presence of benzophenone under an atmosphere of nitrogen. Toluene and dichloroethane were distilled over calcium hydride under an atmosphere of nitrogen. The cyclic diaryliodoniums with triflate anion were synthesized according to the reported literature. ${ }^{1}$

\section{Synthesis of L10}
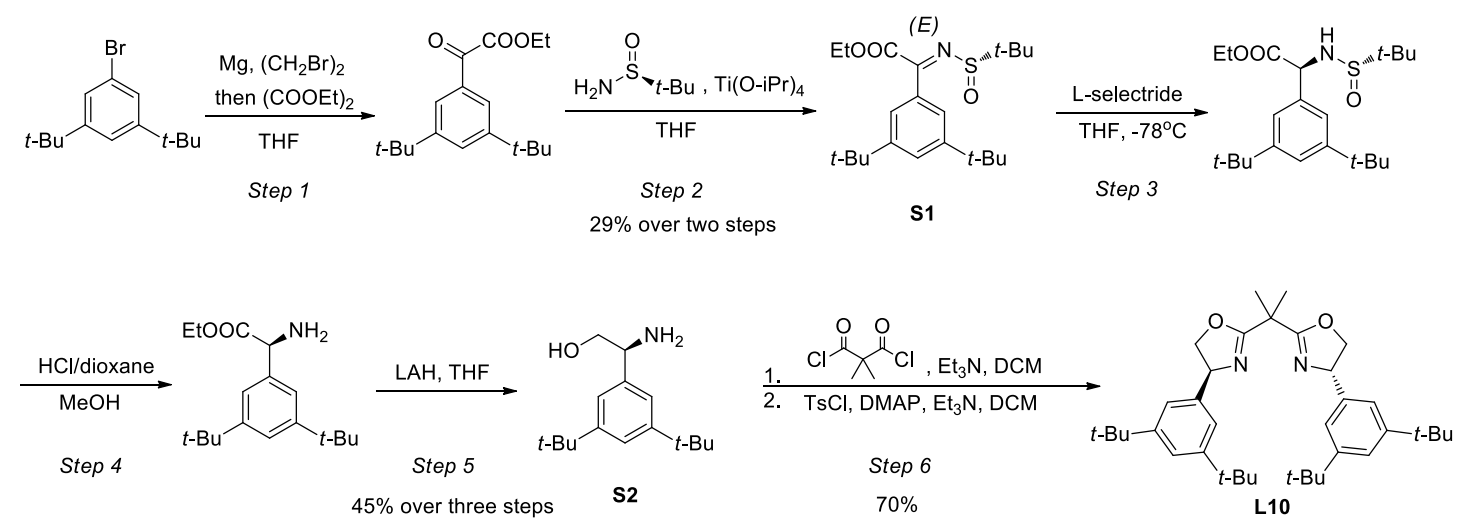

Step 1: Under nitrogen atmosphere, to a $200 \mathrm{~mL}$ Schleck flask containing magnesium (1.89 g, $78 \mathrm{mmol}$, 1.3 equiv) was added anhydrous THF $(30 \mathrm{~mL})$ followed by $\left(\mathrm{CH}_{2} \mathrm{Br}\right)_{2}(0.10 \mathrm{~mL})$. To this mixture, a solution of 1-bromo-3,5-di-tert-butylbenzene (16.15 g, $60 \mathrm{mmol}, 1.0$ equiv) in THF (30 mL) was added dropwise under stirring at room temperature. Then the mixture was stirring at $65{ }^{\circ} \mathrm{C}$ for $1 \mathrm{~h}$. Diethyl 
oxalate $(16.3 \mathrm{~mL}, 120 \mathrm{mmol})$ was dissolved in THF $(30 \mathrm{~mL})$ in a $250 \mathrm{~mL}$ round-bottom flask under nitrogen. The solution was cooled to $-78^{\circ} \mathrm{C}$, then the above Grignard reagent was added to the reaction mixture in $10 \mathrm{~min}$. After stirring for $1 \mathrm{~h}$ at $-78{ }^{\circ} \mathrm{C}$, the reaction was warmed up to room temperature and stirred for another $20 \mathrm{~min}$. After the reaction completed, saturated $\mathrm{NaHCO}_{3}$ solution $(50 \mathrm{~mL})$ was added. After stirring for $5 \mathrm{~min}$, the mixture was filtered through a plug of Celite with ethyl acetate. The filtrate was extracted with ethyl acetate, washed with brine $\left(50 \mathrm{~mL}\right.$ x2), dried over anhydrous $\mathrm{Na}_{2} \mathrm{SO}_{4}$ and concentrated, then $\left(\mathrm{CO}_{2} \mathrm{Et}\right)_{2}$ was removed by distillation at $150{ }^{\circ} \mathrm{C}, 0.01 \mathrm{Mpa}$ to afford the crude product, which was used in next step without further purification.

Step 2: The crude product from Step 1 (11.32 g, about $39 \mathrm{mmol})$ and $(S)$-tert-butylsulfinamide $(4.73 \mathrm{~g}$, $39 \mathrm{mmol})$ were dissolved in anhydrous THF $(60 \mathrm{~mL})$, then $\mathrm{Ti}(\mathrm{O}-\mathrm{iPr})_{4}(17.3 \mathrm{~mL}, 58.5 \mathrm{mmol}, 1.5$ equiv) was added. After stirring at $65^{\circ} \mathrm{C}$ for $12 \mathrm{~h}$ under nitrogen, the solution was cooled to room temperature, diluted with ethyl acetate $(50 \mathrm{~mL})$, quenched by aqueous $\mathrm{NaOH}$ solution $(10 \% \mathrm{w} / \mathrm{w}, 30 \mathrm{~mL})$ and the mixture was stirring for another $10 \mathrm{~min}$ before being filtered through a plug of Celite with ethyl acetate. The filtrate was then washed with brine $(80 \mathrm{~mL}$ x2), dried over anhydrous sodium sulfate, concentrated and purified by flash column chromatography (silica gel, 5\% ethyl acetate in hexanes) to afford the pure $(S)$ - $N$-tert-butanesulfinyl ketimine ester S1 (6.9 g, $17.53 \mathrm{mmol}, 29 \%$ over two steps).

Step 3: In accordance with the literature procedure, ${ }^{2}$ the $(S)$ - $N$-tert-butanesulfinyl ketimine ester $\mathbf{S 1}$ (17.53 mmol, 1.0 equiv) was dissolved in THF $(50 \mathrm{~mL})$ in a $200 \mathrm{~mL}$ round-bottom flask under nitrogen. The solution was cooled to $-78{ }^{\circ} \mathrm{C}$ and stirred for $5 \mathrm{~min}$, then L-Selectride in THF (19.3 mL, $1.0 \mathrm{M}, 19.3 \mathrm{mmol}, 1.1$ equiv) was slowly added to the reaction mixture via a syringe at $-78^{\circ} \mathrm{C}$ over $40 \mathrm{~min}$. The reaction was stirred at $-78{ }^{\circ} \mathrm{C}$ for another $5 \mathrm{~h}$, quenched with saturated $\mathrm{NH}_{4} \mathrm{Cl}$ solution $(30 \mathrm{~mL})$ at $-78^{\circ} \mathrm{C}$, and warmed up to room temperature. The mixture was transferred to a separatory funnel containing brine $(50 \mathrm{~mL})$ and ethyl acetate $(50 \mathrm{~mL})$. The organic layer was separated and the aqueous layer was extracted with ethyl acetate $(50 \mathrm{~mL})$. The combined organic layers were washed with brine $(50 \mathrm{~mL} \times 2)$, dried over sodium sulfate, and concentrated. The crude product was used in the next step without further purification.

Step 4: The crude product from Step 3 was dissolved in methanol $(40 \mathrm{~mL})$ in a $200 \mathrm{~mL}$ round-bottom flask in open air. A solution of hydrogen chloride in dioxane $(17 \mathrm{~mL}, 4.0 \mathrm{M}, 68 \mathrm{mmol})$ was then added to the reaction mixture at $0{ }^{\circ} \mathrm{C}$ over $2 \mathrm{~min}$. The reaction flask was capped with a rubber septum and stirred at room temperature for $1 \mathrm{~h}$. Then aqueous $\mathrm{NaOH}$ solution $(10 \% \mathrm{w} / \mathrm{w}, 30 \mathrm{~mL})$ was added slowly and the solution was extracted with ethyl acetate $(80 \mathrm{~mL})$, washed with brine $(80 \mathrm{~mL} \times 2)$, dried over sodium sulfate, and concentrated to afford the crude product. The ee value (95\% ee) was determined after acetylation of the amino group in accordance with the literature. ${ }^{3}$

Step 5: The crude product from Step 4 was dissolved in anhydrous THF $(50 \mathrm{~mL})$ in a $200 \mathrm{~mL}$ round-bottom flask under nitrogen and the solution was cooled to $0{ }^{\circ} \mathrm{C}$. Lithium aluminum hydride $(2$ 
g, $52.7 \mathrm{mmol}$ ) was then added portionwise. The reaction was stirred at $0{ }^{\circ} \mathrm{C}$ overnight, and carefully quenched by sequential addition of $2 \mathrm{~mL} \mathrm{H} \mathrm{H}_{2} \mathrm{O}-2 \mathrm{~mL} 15 \mathrm{wt} \% \mathrm{NaOH}$ solution $-2 \mathrm{~mL} \mathrm{H}_{2} \mathrm{O}$. The mixture was filtered through Celite with DCM, the filtrate was concentrated and purified by flash column chromatography on silica gel $(\mathrm{DCM} / \mathrm{MeOH}=20 / 1)$ to afford the pure amino alcohol $\mathbf{S 2}$ as white solid (1.98 g, $7.94 \mathrm{mmol}, 29 \%$ over three steps).

Step 6: To an ice-cooled $100 \mathrm{~mL}$ round-bottom flask containing S2 (7.94 mmol, 2.0 equiv), DCM (30 $\mathrm{mL})$ and triethylamine $(2.2 \mathrm{~mL}, 15.88 \mathrm{mmol}, 4.0$ equiv $)$ was added dimethylmalonyl dichloride $(0.525$ $\mathrm{mL}, 3.97 \mathrm{mmol}, 1.0$ equiv) dropwise. After stirring at room temperature for $2 \mathrm{~h}$, tosyl chloride $(1.81 \mathrm{~g}$, $9.53 \mathrm{mmol}, 2.4$ equiv), DMAP (137 mg, $0.794 \mathrm{mmol}, 0.2$ equiv) and triethylamine (2.76 mL, 19.85 mmol, 5.0 equiv) were then added. The reaction was stirred for $2 \mathrm{~d}$ at room temperature before removing the solvent by evaporator, and the residue was purified by flash column chromatography on silica gel (hexanes/ ethyl acetate $=10 / 1)$ to afford $\mathbf{L 1 0}$ as a white solid (1.55 g, $2.77 \mathrm{mmol}, 70 \%)$. $[\alpha]_{D}^{20}=-48\left(\mathrm{c}=1.21, \mathrm{CHCl}_{3}\right) .{ }^{1} \mathbf{H}$ NMR $\left(400 \mathrm{MHz}, \mathrm{CDCl}_{3}\right) \delta 7.32(\mathrm{~d}, J=1.6 \mathrm{~Hz}, 2 \mathrm{H}), 7.10(\mathrm{~d}, J=$ $1.6 \mathrm{~Hz}, 4 \mathrm{H}), 5.17$ (dd, $J=10.0,6.8 \mathrm{~Hz}, 2 \mathrm{H}), 4.64(\mathrm{dd}, J=10.0,8.4 \mathrm{~Hz}, 2 \mathrm{H}), 4.25$ (dd, $J=8.4,6.8 \mathrm{~Hz}$, 2H), 1.69 (s, 6H), 1.29 (s, 36H). ${ }^{13} \mathbf{C}$ NMR (101 MHz, $\left.\mathrm{CDCl}_{3}\right) \delta 170.00,150.98,141.69,121.64$, 121.00, 75.89, 70.10, 38.75, 34.83, 31.41, 24.61. HRMS (ESI): calcd for $\mathrm{C}_{37} \mathrm{H}_{55} \mathrm{~N}_{2} \mathrm{O}_{2}(\mathrm{M}+\mathrm{H})^{+}$ 559.4264 , found 559.4265 .

\section{General Procedure for the Synthesis of 3 (Procedure A)}
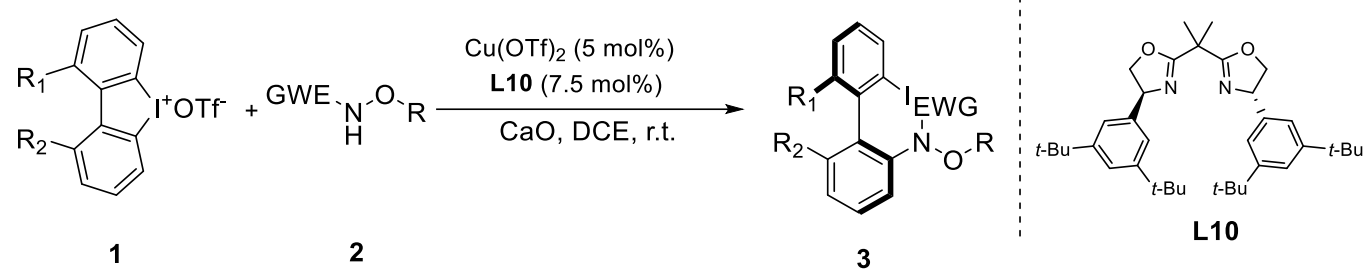

To a Schlenk tube containing 1 (1.0 equiv), 2 (1.2 equiv), $\mathrm{Cu}(\mathrm{OTf})_{2}(5.0 \mathrm{~mol} \%), \mathbf{L 1 0}(7.5 \mathrm{~mol} \%)$ and $\mathrm{CaO}$ (2.0 equiv) was added DCE $(0.05 \mathrm{M})$ under nitrogen at room temperature. After being stirred for 3-12 $\mathrm{h}$ (monitored by TLC) at room temperature, the solvent was removed and the residue was purified by flash column chromatography on silica gel (hexanes/ethyl acetate) to afford the desired product 3.

Compound 3a was prepared following the Procedure A

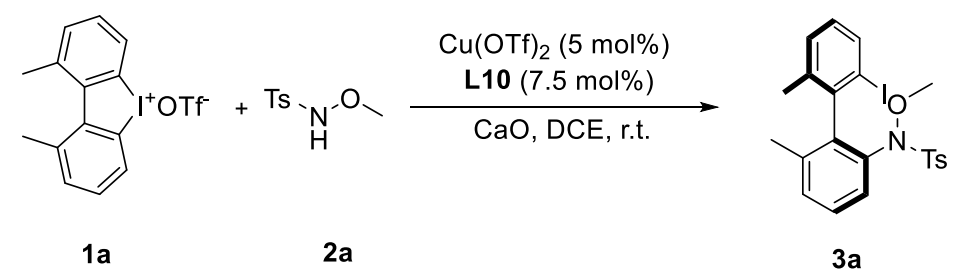

The reaction of $\mathbf{1 a}\left(45.6 \mathrm{mg}, 0.10 \mathrm{mmol}, 1.0\right.$ equiv), $\mathbf{2 a}\left(24.1 \mathrm{mg}, 0.12 \mathrm{mmol}, 1.2\right.$ equiv), $\mathrm{Cu}(\mathrm{OTf})_{2}(1.8$ $\mathrm{mg}, 5.0 \mathrm{~mol} \%), \mathbf{L 1 0}(4.2 \mathrm{mg}, 7.5 \mathrm{~mol} \%)$ and $\mathrm{CaO}(11.2 \mathrm{mg}, 0.20 \mathrm{mmol}, 2.0$ equiv) in DCE (2.0 mL) at 
room temperature for $3 \mathrm{~h}$ afforded $3 \mathrm{a}(50.2 \mathrm{mg}, 99 \%, 99 \% \mathrm{ee}) . \mathrm{Rf}=0.5(\mathrm{PE} / \mathrm{EA}=10: 1) .[\alpha]_{D}^{20}=$ $+105\left(\mathrm{c}=0.93, \mathrm{CHCl}_{3}\right)$. HPLC conditions: Chiralpak AD-H, isopropanol/hexane $=5: 95$, flow: 1.0 $\mathrm{mL} / \mathrm{min}, \lambda=254 \mathrm{~nm} .{ }^{1} \mathbf{H}$ NMR $\left(400 \mathrm{MHz}, \mathrm{CDCl}_{3}\right) \delta 7.77(\mathrm{~d}, J=8.0 \mathrm{~Hz}, 1 \mathrm{H}), 7.60(\mathrm{~d}, J=8.4 \mathrm{~Hz}, 2 \mathrm{H})$, $7.30(\mathrm{dd}, J=12.2,7.8 \mathrm{~Hz}, 4 \mathrm{H}), 7.15(\mathrm{t}, J=7.8 \mathrm{~Hz}, 1 \mathrm{H}), 7.00(\mathrm{t}, J=7.8 \mathrm{~Hz}, 1 \mathrm{H}), 6.63(\mathrm{~d}, J=8.4 \mathrm{~Hz}$, 1H), 3.76 (s, 3H), 2.46 (s, 3H), 2.25 (s, 3H), 2.02 (s, 3H). ${ }^{13} \mathbf{C}$ NMR (101 MHz, $\left.\mathrm{CDCl}_{3}\right) \delta 144.70$, 143.16, 141.67, 139.84, 139.42, 137.69, 136.06, 132.10, 130.88, 130.09, 129.84, 129.16, 129.11, 127.95, 123.69, 101.12, 65.16, 21.78, 21.70, 20.16. HRMS (ESI): calcd for $\mathrm{C}_{22} \mathrm{H}_{22} \mathrm{INO}_{3} \mathrm{SNa}(\mathrm{M}+\mathrm{Na})^{+}$ 530.0263 , found 530.0269 .

Compound 3b was prepared following the Procedure A
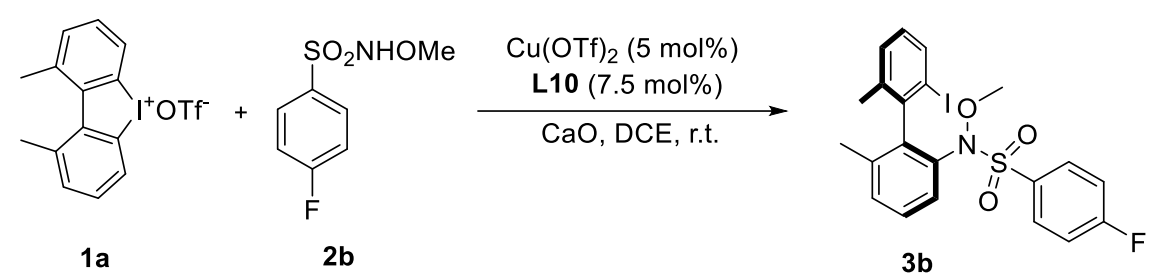

The reaction of $\mathbf{1 a}$ ( $45.6 \mathrm{mg}, 0.10 \mathrm{mmol}, 1.0$ equiv), $\mathbf{2 b}$ ( $24.6 \mathrm{mg}, 0.12 \mathrm{mmol}, 1.2$ equiv), $\mathrm{Cu}(\mathrm{OTf})_{2}(1.8$ $\mathrm{mg}, 5.0 \mathrm{~mol} \%)$, L10 (4.2 mg, $7.5 \mathrm{~mol} \%)$ and $\mathrm{CaO}(11.2 \mathrm{mg}, 0.20 \mathrm{mmol}, 2.0$ equiv) in DCE (2.0 mL) at room temperature for $3 \mathrm{~h}$ afforded $\mathbf{3 b}(51.7 \mathrm{mg},>99 \%, 99 \% \mathrm{ee}) . \mathrm{Rf}=0.5(\mathrm{PE} / \mathrm{EA}=10: 1) .[\alpha]_{D}^{20}=$ $+73\left(\mathrm{c}=0.99, \mathrm{CHCl}_{3}\right)$. HPLC conditions: Chiralpak AD-H, isopropanol/hexane $=5: 95$, flow: 1.0 $\mathrm{mL} / \mathrm{min}, \lambda=254 \mathrm{~nm} .{ }^{1} \mathbf{H} \mathbf{N M R}\left(400 \mathrm{MHz}, \mathrm{CDCl}_{3}\right) \delta 7.77(\mathrm{~d}, J=7.8 \mathrm{~Hz}, 1 \mathrm{H}), 7.75-7.70(\mathrm{~m}, 2 \mathrm{H})$, 7.30 (t, $J=7.2 \mathrm{~Hz}, 2 \mathrm{H}), 7.16$ (q, $J=8.0,7.5 \mathrm{~Hz}, 3 \mathrm{H}), 7.00(\mathrm{t}, J=7.8 \mathrm{~Hz}, 1 \mathrm{H}), 6.56(\mathrm{~d}, J=8.2 \mathrm{~Hz}, 1 \mathrm{H})$, $3.81(\mathrm{~s}, 3 \mathrm{H}), 2.23(\mathrm{~s}, 3 \mathrm{H}), 2.02(\mathrm{~s}, 3 \mathrm{H}) .{ }^{19} \mathbf{F}$ NMR $\left(376 \mathrm{MHz}, \mathrm{CDCl}_{3}\right) \delta-103.30 .{ }^{13} \mathbf{C}$ NMR $(101 \mathrm{MHz}$, $\left.\mathrm{CDCl}_{3}\right) \delta 167.12,164.57,143.07,141.43,139.73,139.15,137.91,136.10,132.84$ (d, $\left.J=9.5 \mathrm{~Hz}\right)$, 131.03, 129.84, 129.22, 127.96, 123.39, 115.78 (d, $J=22.5 \mathrm{~Hz}), 101.04,65.30,21.76,20.17$. HRMS (ESI): calcd for $\mathrm{C}_{21} \mathrm{H}_{19} \mathrm{FINO}_{3} \mathrm{SNa}(\mathrm{M}+\mathrm{Na})^{+} 534.0012$, found 534.0021 .

Compound $\mathbf{3 c}$ was prepared following the Procedure A
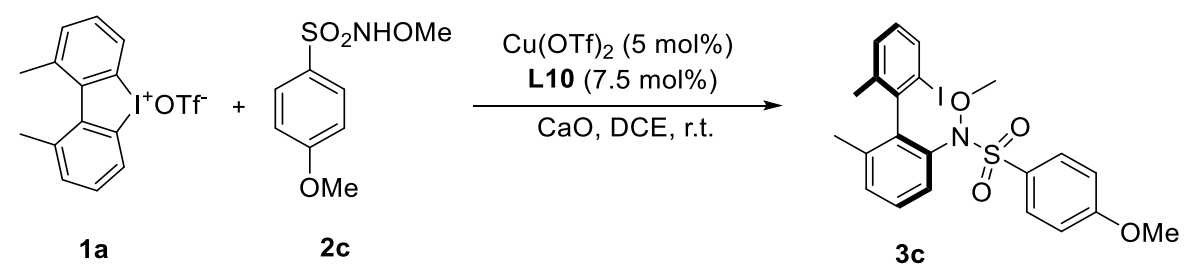

The reaction of $\mathbf{1 a}$ ( $45.6 \mathrm{mg}, 0.10 \mathrm{mmol}, 1.0$ equiv), $\mathbf{2 c}$ ( $26.1 \mathrm{mg}, 0.12 \mathrm{mmol}, 1.2$ equiv), $\mathrm{Cu}(\mathrm{OTf})_{2}(1.8$ $\mathrm{mg}, 5.0 \mathrm{~mol} \%), \mathbf{L 1 0}(4.2 \mathrm{mg}, 7.5 \mathrm{~mol} \%)$ and $\mathrm{CaO}(11.2 \mathrm{mg}, 0.20 \mathrm{mmol}, 2.0$ equiv) in DCE (2.0 mL) at room temperature for $3 \mathrm{~h}$ afforded $3 \mathrm{c}(54.1 \mathrm{mg},>99 \%, 99 \% \mathrm{ee})$. $\mathrm{Rf}=0.4(\mathrm{PE} / \mathrm{EA}=10: 1) .[\alpha]_{D}^{20}=$ $+106\left(\mathrm{c}=0.98, \mathrm{CHCl}_{3}\right)$. HPLC conditions: Chiralpak AD-H, isopropanol/hexane $=5: 95$, flow: 1.0 $\mathrm{mL} / \mathrm{min}, \lambda=254 \mathrm{~nm} .{ }^{1} \mathbf{H}$ NMR $\left(400 \mathrm{MHz}, \mathrm{CDCl}_{3}\right) \delta 7.77(\mathrm{~d}, J=8.0 \mathrm{~Hz}, 1 \mathrm{H}), 7.68-7.62(\mathrm{~m}, 2 \mathrm{H})$, $7.30(\mathrm{dd}, J=11.8,7.6 \mathrm{~Hz}, 2 \mathrm{H}), 7.16(\mathrm{t}, J=7.8 \mathrm{~Hz}, 1 \mathrm{H}), 7.00(\mathrm{t}, J=7.6 \mathrm{~Hz}, 1 \mathrm{H}), 6.97-6.93(\mathrm{~m}, 2 \mathrm{H})$, $6.63(\mathrm{~d}, J=8.0 \mathrm{~Hz}, 1 \mathrm{H}), 3.89$ (s, 3H), 3.77 (s, 3H), 2.25 (s, 3H), 2.02 (s, 3H). ${ }^{13} \mathbf{C}$ NMR (101 MHz, $\left.\mathrm{CDCl}_{3}\right) \delta 163.77,143.09,141.67,139.79,139.57,137.63,136.03,132.22,130.81,129.80,129.12$, 127.90, 126.39, 123.61, 113.64, 101.12, 65.16, 55.62, 21.76, 20.14. HRMS (ESI): calcd for $\mathrm{C}_{22} \mathrm{H}_{22} \mathrm{INO}_{4} \mathrm{SNa}(\mathrm{M}+\mathrm{Na})^{+} 546.0212$, found 546.0212 . 
Compound 3d was prepared following the Procedure A

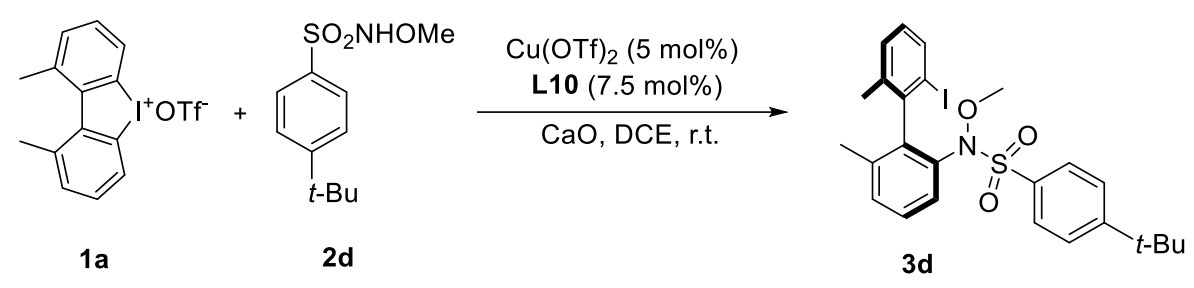

The reaction of $\mathbf{1 a}$ ( $45.6 \mathrm{mg}, 0.10 \mathrm{mmol}, 1.0$ equiv), $\mathbf{2 d}\left(29.2 \mathrm{mg}, 0.12 \mathrm{mmol}, 1.2\right.$ equiv), $\mathrm{Cu}(\mathrm{OTf})_{2}(1.8$ $\mathrm{mg}, 5.0 \mathrm{~mol} \%), \mathbf{L 1 0}(4.2 \mathrm{mg}, 7.5 \mathrm{~mol} \%)$ and $\mathrm{CaO}(11.2 \mathrm{mg}, 0.20 \mathrm{mmol}, 2.0$ equiv) in DCE (2.0 mL) at room temperature for $3 \mathrm{~h}$ afforded $\mathbf{3 d}(54.7 \mathrm{mg}, 99 \%, 99 \% \mathrm{ee}) . \mathrm{Rf}=0.5(\mathrm{PE} / \mathrm{EA}=10: 1) .[\alpha]_{D}^{20}=+82$ (c $\left.=0.78, \mathrm{CHCl}_{3}\right)$. HPLC conditions: Chiralpak AD-H, isopropanol/hexane $=5: 95$, flow: $1.0 \mathrm{~mL} / \mathrm{min}, \lambda$ $=254 \mathrm{~nm} .{ }^{1} \mathbf{H}$ NMR $\left(400 \mathrm{MHz}, \mathrm{CDCl}_{3}\right) \delta 7.77(\mathrm{~d}, J=8.0 \mathrm{~Hz}, 1 \mathrm{H}), 7.67-7.63(\mathrm{~m}, 2 \mathrm{H}), 7.52-7.47(\mathrm{~m}$, 2H), $7.30(\mathrm{dd}, J=12.4,7.6 \mathrm{~Hz}, 2 \mathrm{H}), 7.14(\mathrm{t}, J=7.8 \mathrm{~Hz}, 1 \mathrm{H}), 7.00(\mathrm{t}, J=7.8 \mathrm{~Hz}, 1 \mathrm{H}), 6.63(\mathrm{~d}, J=8.0$ $\mathrm{Hz}, 1 \mathrm{H}), 3.75$ (s, 3H), 2.25 (s, 3H), $2.02(\mathrm{~s}, 3 \mathrm{H}), 1.36(\mathrm{~s}, 9 \mathrm{H}) .{ }^{13} \mathbf{C}$ NMR $\left(101 \mathrm{MHz}, \mathrm{CDCl}_{3}\right) \delta 157.71$, 143.22 , 141.69, 139.80, 139.38, 137.71, 136.07, 132.07, 130.91, 129.92, 129.85, 129.18, 127.93, 125.45, 123.73, 101.16, 65.14, 35.26, 31.07, 21.78, 20.16. HRMS (ESI): calcd for $\mathrm{C}_{25} \mathrm{H}_{28} \mathrm{INO}_{3} \mathrm{SNa}$ $(\mathrm{M}+\mathrm{Na})^{+}$572.0732, found 572.0736.

Compound 3e was prepared following the Procedure A
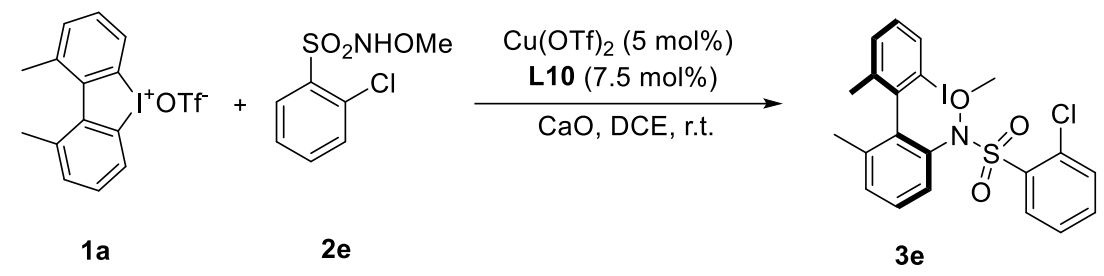

The reaction of $\mathbf{1 a}\left(45.6 \mathrm{mg}, 0.10 \mathrm{mmol}, 1.0\right.$ equiv), $\mathbf{2 e}\left(26.6 \mathrm{mg}, 0.12 \mathrm{mmol}, 1.2\right.$ equiv), $\mathrm{Cu}(\mathrm{OTf})_{2}(1.8$ $\mathrm{mg}, 5.0 \mathrm{~mol} \%), \mathbf{L 1 0}(4.2 \mathrm{mg}, 7.5 \mathrm{~mol} \%)$ and $\mathrm{CaO}(11.2 \mathrm{mg}, 0.20 \mathrm{mmol}, 2.0$ equiv) in DCE (2.0 mL) at room temperature for $12 \mathrm{~h}$ afforded $3 \mathrm{e}(45.7 \mathrm{mg}, 86 \%, 88 \% \mathrm{ee}) . \mathrm{Rf}=0.4(\mathrm{PE} / \mathrm{EA}=10: 1) .[\alpha]_{D}^{20}=$ $+37.6\left(\mathrm{c}=0.85, \mathrm{CHCl}_{3}\right)$. HPLC conditions: Chiralpak AD-H, isopropanol/hexane = 5:95, flow: 1.0 $\mathrm{mL} / \mathrm{min}, \lambda=254 \mathrm{~nm} .{ }^{1} \mathbf{H} \mathbf{N M R}\left(400 \mathrm{MHz}, \mathrm{CDCl}_{3}\right) \delta 8.03(\mathrm{dd}, J=8.0,1.6 \mathrm{~Hz}, 1 \mathrm{H}), 7.76(\mathrm{~d}, J=7.6 \mathrm{~Hz}$, $1 \mathrm{H}), 7.53(\mathrm{ddd}, J=8.0,7.2,1.6 \mathrm{~Hz}, 1 \mathrm{H}), 7.47(\mathrm{dd}, J=8.0,1.4 \mathrm{~Hz}, 1 \mathrm{H}), 7.41-7.33$ (m, 2H), $7.32-$ $7.27(\mathrm{~m}, 2 \mathrm{H}), 7.26-7.22(\mathrm{~m}, 1 \mathrm{H}), 6.99(\mathrm{t}, J=7.8 \mathrm{~Hz}, 1 \mathrm{H}), 3.55(\mathrm{~s}, 3 \mathrm{H}), 2.23(\mathrm{~s}, 3 \mathrm{H}), 2.01(\mathrm{~s}, 3 \mathrm{H}) .{ }^{13} \mathrm{C}$ NMR $\left(101 \mathrm{MHz}, \mathrm{CDCl}_{3}\right) \delta 143.12,141.74,140.35,138.31,137.80,136.07,134.76,134.65,134.25$, 133.63, 132.09, 131.32, 129.99, 129.16, 128.28, 126.62, 125.02, 101.12, 64.52, 21.86, 20.21. HRMS (ESI): calcd for $\mathrm{C}_{21} \mathrm{H}_{19} \mathrm{ClINO}_{3} \mathrm{SNa}(\mathrm{M}+\mathrm{Na})^{+} 549.9717$, found 549.9711 .

Compound 3f was prepared following the Procedure A

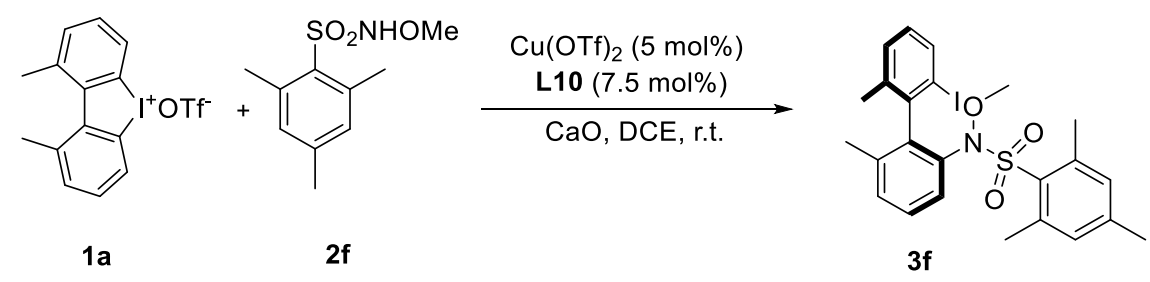


The reaction of $\mathbf{1 a}\left(45.6 \mathrm{mg}, 0.10 \mathrm{mmol}, 1.0\right.$ equiv), $\mathbf{2 f}\left(27.5 \mathrm{mg}, 0.12 \mathrm{mmol}, 1.2\right.$ equiv), $\mathrm{Cu}(\mathrm{OTf})_{2}(1.8$ $\mathrm{mg}, 5.0 \mathrm{~mol} \%), \mathbf{L 1 0}(4.2 \mathrm{mg}, 7.5 \mathrm{~mol} \%)$ and $\mathrm{CaO}(11.2 \mathrm{mg}, 0.20 \mathrm{mmol}, 2.0$ equiv) in DCE (2.0 mL) at room temperature for $3 \mathrm{~h}$ afforded $3 \mathbf{f}(50.1 \mathrm{mg}, 94 \%, 96 \%$ ee $) . \mathrm{Rf}=0.5(\mathrm{PE} / \mathrm{EA}=10: 1) .[\alpha]_{D}^{20}=$ $+22.7\left(\mathrm{c}=0.74, \mathrm{CHCl}_{3}\right)$. HPLC conditions: Chiralpak AD-H, isopropanol/hexane = 5:95, flow: 1.0 $\mathrm{mL} / \mathrm{min}, \lambda=254 \mathrm{~nm} .{ }^{1} \mathbf{H}$ NMR $\left(400 \mathrm{MHz}, \mathrm{CDCl}_{3}\right) \delta 7.76-7.68(\mathrm{~m}, 2 \mathrm{H}), 7.42-7.33(\mathrm{~m}, 2 \mathrm{H}), 7.26(\mathrm{~d}$, $J=8.0 \mathrm{~Hz}, 1 \mathrm{H}), 6.96(\mathrm{t}, J=7.6 \mathrm{~Hz}, 1 \mathrm{H}), 6.89(\mathrm{~s}, 2 \mathrm{H}), 3.23(\mathrm{~s}, 3 \mathrm{H}), 2.31(\mathrm{~s}, 6 \mathrm{H}), 2.28(\mathrm{~s}, 3 \mathrm{H}), 2.22(\mathrm{~s}$, 3H), $2.00(\mathrm{~s}, 3 \mathrm{H}) .{ }^{13} \mathbf{C}$ NMR $\left(101 \mathrm{MHz}, \mathrm{CDCl}_{3}\right) \delta 143.50,142.73,142.51,142.15,140.56,138.69$, 137.23, 136.06, 131.69, 131.04, 130.97, 130.17, 129.06, 128.32, 126.10, 101.02, 63.80, 22.55, 21.91, 21.03, 20.21. HRMS (ESI): calcd for $\mathrm{C}_{24} \mathrm{H}_{26} \mathrm{INO}_{3} \mathrm{SNa}(\mathrm{M}+\mathrm{Na})^{+} 558.0576$, found 558.0583.

Compound $\mathbf{3 g}$ was prepared following the Procedure A

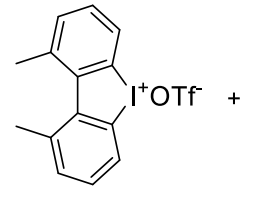

$1 \mathrm{a}$

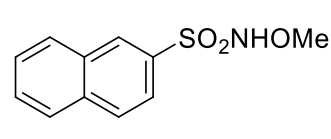

$2 \mathrm{~g}$

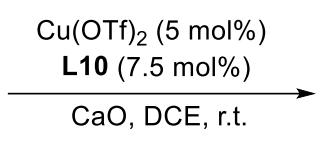
The reaction of $\mathbf{1 a}\left(45.6 \mathrm{mg}, 0.10 \mathrm{mmol}, 1.0\right.$ equiv), $\mathbf{2 g}\left(28.5 \mathrm{mg}, 0.12 \mathrm{mmol}, 1.2\right.$ equiv), $\mathrm{Cu}(\mathrm{OTf})_{2}(1.8$
$\mathrm{mg}, 5.0 \mathrm{~mol} \%), \mathbf{L 1 0}(4.2 \mathrm{mg}, 7.5 \mathrm{~mol} \%)$ and $\mathrm{CaO}(11.2 \mathrm{mg}, 0.20 \mathrm{mmol}, 2.0$ equiv) in DCE $(2.0 \mathrm{~mL})$ at room temperature for $3 \mathrm{~h}$ afforded $\mathbf{3 g}(\mathrm{mg}, 93 \%, 94 \% \mathrm{ee}) . \mathrm{Rf}=0.5(\mathrm{PE} / \mathrm{EA}=10: 1) .[\alpha]_{D}^{20}=+83.6(\mathrm{c}$ $\left.=1.07, \mathrm{CHCl}_{3}\right)$. HPLC conditions: Chiralpak AD-H, isopropanol $/$ hexane $=5: 95$, flow: $1.0 \mathrm{~mL} / \mathrm{min}, \lambda=$ $254 \mathrm{~nm} .{ }^{1} \mathbf{H}$ NMR $\left(400 \mathrm{MHz}, \mathrm{CDCl}_{3}\right) \delta 8.32(\mathrm{~s}, 1 \mathrm{H}), 7.97-7.88(\mathrm{~m}, 3 \mathrm{H}), 7.79(\mathrm{~d}, J=7.2 \mathrm{~Hz}, 1 \mathrm{H})$, $7.71-7.59(\mathrm{~m}, 3 \mathrm{H}), 7.35(\mathrm{~d}, J=7.6 \mathrm{~Hz}, 1 \mathrm{H}), 7.29(\mathrm{~d}, J=7.6 \mathrm{~Hz}, 1 \mathrm{H}), 7.10(\mathrm{t}, J=7.8 \mathrm{~Hz}, 1 \mathrm{H}), 7.03(\mathrm{t}$, $J=7.8 \mathrm{~Hz}, 1 \mathrm{H}), 6.68(\mathrm{~d}, J=8.0 \mathrm{~Hz}, 1 \mathrm{H}), 3.75(\mathrm{~s}, 3 \mathrm{H}), 2.30(\mathrm{~s}, 3 \mathrm{H}), 2.04(\mathrm{~s}, 3 \mathrm{H}) .{ }^{13} \mathbf{C ~ N M R}(101 \mathrm{MHz}$, $\left.\mathrm{CDCl}_{3}\right) \delta 143.19,141.68,139.85,139.13,137.77,136.10,135.28,132.39,131.75,131.01,129.86$, $129.44,129.20,128.43,128.03,127.87,127.40,124.79,123.90,101.16,65.15,21.82,20.15$. HRMS (ESI): calcd for $\mathrm{C}_{25} \mathrm{H}_{22} \mathrm{INO}_{3} \mathrm{SNa}(\mathrm{M}+\mathrm{Na})^{+} 566.0263$, found 566.0270.

Compound 3h was prepared following the Procedure A

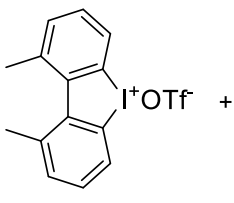

$1 a$

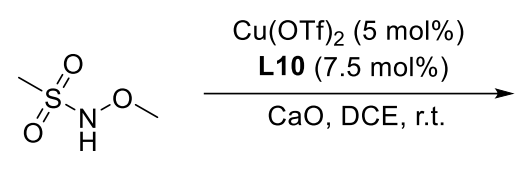

$2 h$

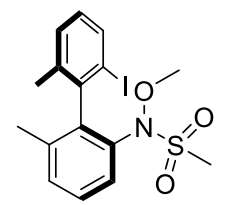

$3 h$

The reaction of $\mathbf{1 a}$ (45.6 mg, $0.10 \mathrm{mmol}, 1.0$ equiv), $\mathbf{2 h}$ (15.0 mg, $0.12 \mathrm{mmol}, 1.2$ equiv), $\mathrm{Cu}(\mathrm{OTf})_{2}(1.8$ $\mathrm{mg}, 5.0 \mathrm{~mol} \%), \mathbf{L 1 0}(4.2 \mathrm{mg}, 7.5 \mathrm{~mol} \%)$ and $\mathrm{CaO}(11.2 \mathrm{mg}, 0.20 \mathrm{mmol}, 2.0$ equiv) in DCE (2.0 mL) at room temperature for $3 \mathrm{~h}$ afforded $3 \mathrm{~h}(37.9 \mathrm{mg}, 88 \%, 96 \%$ ee $) . \mathrm{Rf}=0.5(\mathrm{PE} / \mathrm{EA}=10: 1) .[\alpha]_{D}^{20}=$ +49.5 $\left(\mathrm{c}=1.10, \mathrm{CHCl}_{3}\right)$. HPLC conditions: Chiralpak AD-H, isopropanol/hexane = 5:95, flow: 1.0 $\mathrm{mL} / \mathrm{min}, \lambda=254 \mathrm{~nm} .{ }^{1} \mathbf{H} \mathbf{N M R}\left(400 \mathrm{MHz}, \mathrm{CDCl}_{3}\right) \delta 7.75(\mathrm{~d}, J=8.0 \mathrm{~Hz}, 1 \mathrm{H}), 7.41$ (dd, $J=7.2,5.6 \mathrm{~Hz}$, 2H), $7.37-7.34(\mathrm{~m}, 1 \mathrm{H}), 7.27-7.24(\mathrm{~m}, 1 \mathrm{H}), 6.97$ (t, J=7.8 Hz, 1H), 3.85 (s, 3H), 3.00 (s, 3H), 2.12 (s, 3H), $2.01(\mathrm{~s}, 3 \mathrm{H}) .{ }^{13} \mathrm{C}$ NMR $\left(101 \mathrm{MHz}, \mathrm{CDCl}_{3}\right) \delta 143.33,141.47,139.57,138.51,138.36,136.12$, $131.17,129.84,129.25,128.41,123.45,100.97,64.97,34.68,21.62,20.14$. HRMS (ESI): calcd for $\mathrm{C}_{16} \mathrm{H}_{18} \mathrm{INO}_{3} \mathrm{SNa}(\mathrm{M}+\mathrm{Na})^{+} 453.9950$, found 453.9955 . 
Compound 3i was prepared following the Procedure A

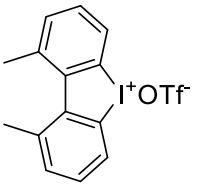

$1 \mathbf{a}$

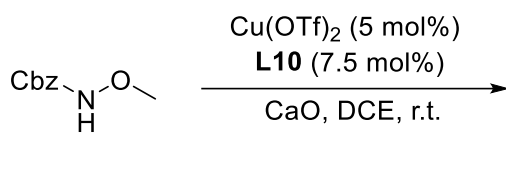

$\mathbf{2 i}$

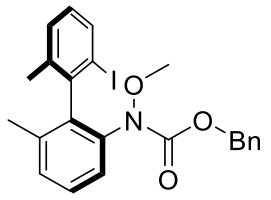

$3 \mathbf{i}$

The reaction of $1 \mathbf{a}$ ( $45.6 \mathrm{mg}, 0.10 \mathrm{mmol}, 1.0$ equiv), $2 \mathbf{i}\left(21.7 \mathrm{mg}, 0.12 \mathrm{mmol}, 1.2\right.$ equiv), $\mathrm{Cu}(\mathrm{OTf})_{2}(1.8$ $\mathrm{mg}, 5.0 \mathrm{~mol} \%)$, L10 (4.2 mg, $7.5 \mathrm{~mol} \%)$ and $\mathrm{CaO}(11.2 \mathrm{mg}, 0.20 \mathrm{mmol}, 2.0$ equiv) in DCE (2.0 mL) at room temperature for $6 \mathrm{~h}$ afforded $3 \mathbf{i}(42.1 \mathrm{mg}, 86 \%, 93 \% \mathrm{ee}) . \mathrm{Rf}=0.5(\mathrm{PE} / \mathrm{EA}=10: 1) . \quad[\alpha]_{D}^{20}=+0.7$ $\left(\mathrm{c}=0.89, \mathrm{CHCl}_{3}\right)$. HPLC conditions: Chiralpak AD-H, isopropanol/hexane $=10: 90$, flow: $1.0 \mathrm{~mL} / \mathrm{min}$, $\lambda=254 \mathrm{~nm} .{ }^{1} \mathbf{H}$ NMR $\left(400 \mathrm{MHz}, \mathrm{CDCl}_{3}\right) \delta 7.74(\mathrm{~d}, J=7.5 \mathrm{~Hz}, 1 \mathrm{H}), 7.35-7.26(\mathrm{~m}, 7 \mathrm{H}), 7.19(\mathrm{~d}, J=$ $7.2 \mathrm{~Hz}, 1 \mathrm{H}), 7.15(\mathrm{t}, J=4.6 \mathrm{~Hz}, 1 \mathrm{H}), 6.93(\mathrm{t}, J=7.6 \mathrm{~Hz}, 1 \mathrm{H}), 5.19(\mathrm{~d}, J=12.4 \mathrm{~Hz}, 1 \mathrm{H}), 5.16(\mathrm{~d}, J=$ $12.4 \mathrm{~Hz}, 1 \mathrm{H}), 3.48$ (s, 3H), 2.00 (s, 3H), 1.94 (s, 3H). ${ }^{13} \mathbf{C}$ NMR (101 MHz, CDCl 3 ) $\delta 155.20,142.22$, $141.98,138.25,137.92,136.81,136.39,135.99,130.69,129.68,129.10,128.37,128.02,127.97$, 127.91, 126.67, 101.59, 67.52, 61.85, 21.15, 19.79. HRMS (ESI): calcd for $\mathrm{C}_{23} \mathrm{H}_{22} \mathrm{INO}_{3} \mathrm{Na}(\mathrm{M}+\mathrm{Na})^{+}$ 510.0542 , found 510.0535 .

Compound $\mathbf{3} \mathbf{j}$ was prepared following the Procedure A

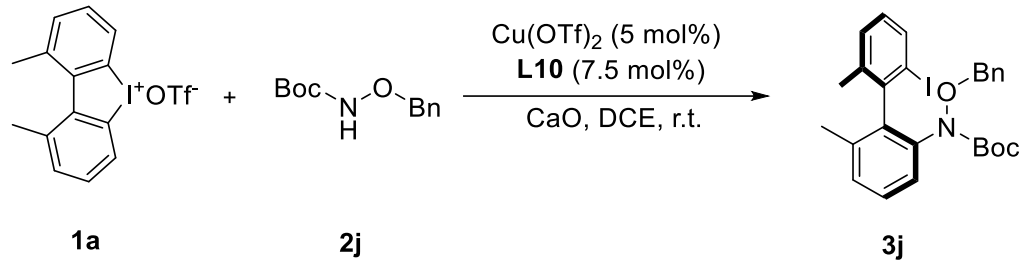

The reaction of $\mathbf{1 a}$ ( $45.6 \mathrm{mg}, 0.10 \mathrm{mmol}, 1.0$ equiv), $\mathbf{2 j}$ ( $26.8 \mathrm{mg}, 0.12 \mathrm{mmol}, 1.2$ equiv), $\mathrm{Cu}(\mathrm{OTf})_{2}(1.8$ $\mathrm{mg}, 5.0 \mathrm{~mol} \%), \mathbf{L 1 0}(4.2 \mathrm{mg}, 7.5 \mathrm{~mol} \%)$ and $\mathrm{CaO}(11.2 \mathrm{mg}, 0.20 \mathrm{mmol}, 2.0$ equiv) in DCE (2.0 mL) at room temperature for $12 \mathrm{~h}$ afforded $3 \mathbf{j}\left(44.1 \mathrm{mg}, 83 \%, 95 \%\right.$ ee). Rf $=0.6(\mathrm{PE} / \mathrm{EA}=20: 1) .[\alpha]_{D}^{20}=$ $+9.5\left(\mathrm{c}=1.01, \mathrm{CHCl}_{3}\right)$. HPLC conditions: Chiralpak OD-H, isopropanol/hexane $=0.5: 99.5$, flow: 1.0 $\mathrm{mL} / \mathrm{min}, \lambda=254 \mathrm{~nm} .{ }^{1} \mathbf{H}$ NMR $\left(400 \mathrm{MHz}, \mathrm{CDCl}_{3}\right) \delta 7.77(\mathrm{~d}, J=8.0 \mathrm{~Hz}, 1 \mathrm{H}), 7.39-7.26(\mathrm{~m}, 7 \mathrm{H})$, $7.23(\mathrm{~d}, J=7.6 \mathrm{~Hz}, 1 \mathrm{H}), 7.16(\mathrm{~d}, J=7.2 \mathrm{~Hz}, 1 \mathrm{H}), 6.95(\mathrm{t}, J=8.0 \mathrm{~Hz}, 1 \mathrm{H}), 4.54(\mathrm{~d}, J=9.4 \mathrm{~Hz}, 1 \mathrm{H})$, $4.50(\mathrm{~d}, J=9.4 \mathrm{~Hz}, 1 \mathrm{H}), 2.04$ (s, 3H), $2.01(\mathrm{~s}, 3 \mathrm{H}), 1.43$ (s, 9H). ${ }^{13} \mathbf{C} \mathbf{~ N M R}\left(101 \mathrm{MHz}, \mathrm{CDCl}_{3}\right) \delta 155.18$, $142.41,142.34,138.66,138.51,137.59$, 136.40, 135.48, 130.24, 129.68, 129.50, 129.06, 128.10, $128.05,127.97,126.84,101.80,81.63,75.96,28.22,21.34,19.91$. HRMS (ESI): calcd for $\mathrm{C}_{26} \mathrm{H}_{28} \mathrm{INO}_{3} \mathrm{Na}(\mathrm{M}+\mathrm{Na})^{+}$552.1012, found 552.1016.

A reaction at $1 \mathbf{~ m m o l ~ s c a l e : ~ T o ~ a ~ S c h l e n k ~ t u b e ~ c o n t a i n i n g ~} \mathbf{1 a}(0.456 \mathrm{~g}, 1.0 \mathrm{mmol}, 1.0$ equiv), $\mathbf{2 j}$ ( 0.268 $\mathrm{g}, 1.2 \mathrm{mmol}, 1.2$ equiv), $\mathrm{Cu}(\mathrm{OTf})_{2}(18 \mathrm{mg}, 5.0 \mathrm{~mol} \%), \mathbf{L 1 0}(42 \mathrm{mg}, 7.5 \mathrm{~mol} \%)$ and $\mathrm{CaO}(0.112 \mathrm{~g}, 2.0$ mmol, 2.0 equiv) was added DCE $(10.0 \mathrm{~mL}, 0.10 \mathrm{M}$ of 1a) under nitrogen at room temperature and stirred at the same temperature. After complete consumption of starting material $(12 \mathrm{~h}$ at room temperature), the solvent was removed by evaporation and the residue was purified by flash column chromatography on silica gel (hexanes/ethyl acetate $=40: 1)$ to afford the desired product $\mathbf{3 j}$ ( $0.481 \mathrm{~g}$, $91 \%, 95 \%$ ee).

Compound 3k was prepared following the Procedure A 

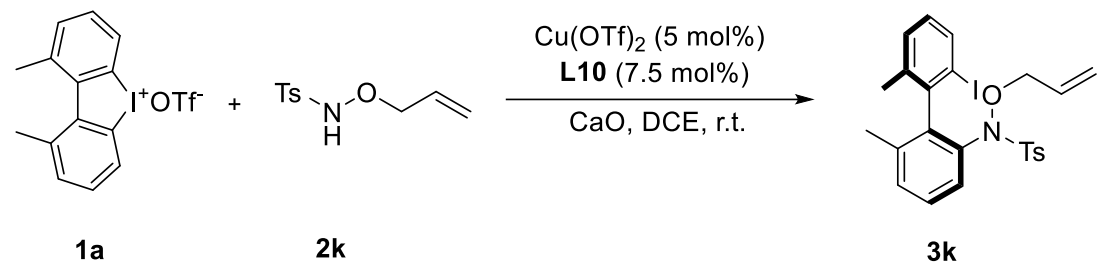

The reaction of $\mathbf{1 a}\left(91.2 \mathrm{mg}, 0.20 \mathrm{mmol}, 1.0\right.$ equiv), $\mathbf{2 k}\left(54.5 \mathrm{mg}, 0.24 \mathrm{mmol}, 1.2\right.$ equiv), $\mathrm{Cu}(\mathrm{OTf})_{2}(3.6$ $\mathrm{mg}, 5.0 \mathrm{~mol} \%), \mathbf{L 1 0}(8.4 \mathrm{mg}, 7.5 \mathrm{~mol} \%)$ and $\mathrm{CaO}(22.4 \mathrm{mg}, 0.40 \mathrm{mmol}, 2.0$ equiv) in DCE (4.0 mL) at room temperature for $6 \mathrm{~h}$ afforded $3 \mathbf{k}(100.5 \mathrm{mg}, 94 \%, 75 \% \mathrm{ee}) . \mathrm{Rf}=0.4(\mathrm{PE} / \mathrm{EA}=10: 1) .[\alpha]_{D}^{20}=$ $+23.3\left(\mathrm{c}=0.92, \mathrm{CHCl}_{3}\right)$. HPLC conditions: Chiralpak AD-H, isopropanol/hexane = 5:95, flow: 1.0 $\mathrm{mL} / \mathrm{min}, \lambda=254 \mathrm{~nm} .{ }^{1} \mathbf{H}$ NMR $\left(400 \mathrm{MHz}, \mathrm{CDCl}_{3}\right) \delta 7.80-7.73(\mathrm{~m}, 1 \mathrm{H}), 7.59(\mathrm{~d}, J=8.4 \mathrm{~Hz}, 2 \mathrm{H})$, $7.34-7.26(\mathrm{~m}, 4 \mathrm{H}), 7.13(\mathrm{t}, J=8.0 \mathrm{~Hz}, 1 \mathrm{H}), 7.00(\mathrm{t}, J=7.6 \mathrm{~Hz}, 1 \mathrm{H}), 6.60(\mathrm{~d}, J=7.6 \mathrm{~Hz}, 1 \mathrm{H}), 5.84$ (ddt, $J=16.8,10.4,6.2 \mathrm{~Hz}, 1 \mathrm{H}), 5.29-5.13(\mathrm{~m}, 2 \mathrm{H}), 4.70$ (ddt, $J=11.0,6.0,1.2 \mathrm{~Hz}, 1 \mathrm{H}), 4.39$ (ddt, $J$ $=10.9,6.4,1.2 \mathrm{~Hz}, 1 \mathrm{H}), 2.46(\mathrm{~s}, 3 \mathrm{H}), 2.26(\mathrm{~s}, 3 \mathrm{H}), 2.01(\mathrm{~s}, 3 \mathrm{H}) .{ }^{13} \mathbf{C ~ N M R}\left(101 \mathrm{MHz}, \mathrm{CDCl}_{3}\right) \delta 144.71$, $143.06,141.59,139.93,139.60,137.60,136.04,132.24,131.68,130.82,130.27,129.87,129.17$, 129.06, 127.91, 123.79, 119.35, 101.06, 78.44, 21.81, 21.71, 20.17. HRMS (ESI): calcd for $\mathrm{C}_{24} \mathrm{H}_{24} \mathrm{INO}_{3} \mathrm{SNa}(\mathrm{M}+\mathrm{Na})^{+} 556.0419$, found 556.0419.

Compound 31 was prepared following the Procedure A
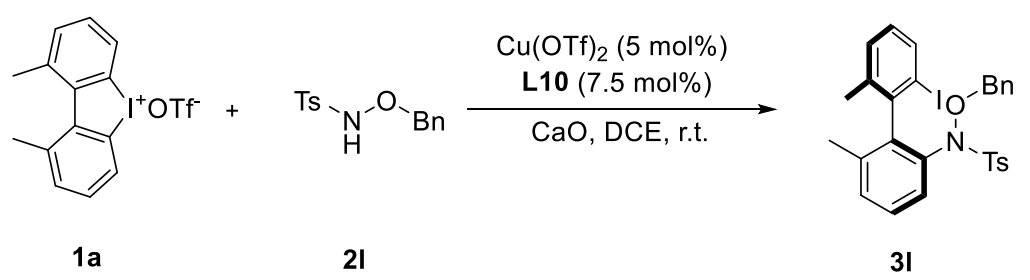

The reaction of $\mathbf{1 a}\left(45.6 \mathrm{mg}, 0.10 \mathrm{mmol}, 1.0\right.$ equiv), $2 \mathrm{l}$ (33.3 mg, $0.12 \mathrm{mmol}, 1.2$ equiv), $\mathrm{Cu}(\mathrm{OTf})_{2}(1.8$ $\mathrm{mg}, 5.0 \mathrm{~mol} \%), \mathbf{L 1 0}(4.2 \mathrm{mg}, 7.5 \mathrm{~mol} \%)$ and $\mathrm{CaO}(11.2 \mathrm{mg}, 0.20 \mathrm{mmol}, 2.0$ equiv) in DCE (2.0 mL) at room temperature for $6 \mathrm{~h}$ afforded $31(61.8 \mathrm{mg},>99 \%, 98 \%$ ee $) . \mathrm{Rf}=0.5(\mathrm{PE} / \mathrm{EA}=10: 1) .[\alpha]_{D}^{20}=$ -3.3 (c = 1.06, $\left.\mathrm{CHCl}_{3}\right)$. HPLC conditions: Chiralpak AD-H, isopropanol/hexane = 5:95, flow: 1.0 $\mathrm{mL} / \mathrm{min}, \lambda=254 \mathrm{~nm} .{ }^{1} \mathbf{H}$ NMR $\left(400 \mathrm{MHz}, \mathrm{CDCl}_{3}\right) \delta 7.80(\mathrm{~d}, J=7.6 \mathrm{~Hz}, 1 \mathrm{H}), 7.58(\mathrm{~d}, J=8.4 \mathrm{~Hz}, 2 \mathrm{H})$, $7.38-7.28(\mathrm{~m}, 7 \mathrm{H}), 7.24(\mathrm{~d}, J=8.0 \mathrm{~Hz}, 2 \mathrm{H}), 7.12(\mathrm{t}, J=7.8 \mathrm{~Hz}, 1 \mathrm{H}), 7.03(\mathrm{t}, J=7.8 \mathrm{~Hz}, 1 \mathrm{H}), 6.58(\mathrm{~d}$, $J=8.0 \mathrm{~Hz}, 1 \mathrm{H}), 5.34(\mathrm{~d}, J=9.2 \mathrm{~Hz}, 1 \mathrm{H}), 4.95(\mathrm{~d}, J=9.6 \mathrm{~Hz}, 1 \mathrm{H}), 2.43(\mathrm{~s}, 3 \mathrm{H}), 2.31(\mathrm{~s}, 3 \mathrm{H}), 2.05$ (s, $3 \mathrm{H}) .{ }^{13} \mathrm{C}$ NMR $\left(101 \mathrm{MHz}, \mathrm{CDCl}_{3}\right) \delta 144.63,142.94,141.59,140.10,139.72,137.59,136.10,135.46$, $131.44,130.76,130.31,129.90,129.19,129.16,129.02$, 128.25, 127.84, 123.92, 101.12, 79.19, 21.88, 21.65, 20.20. HRMS (ESI): calcd for $\mathrm{C}_{28} \mathrm{H}_{26} \mathrm{INO}_{3} \mathrm{SNa}(\mathrm{M}+\mathrm{Na})^{+} 606.0576$, found 606.0579.

Compound $\mathbf{3 m}$ was prepared following the Procedure A
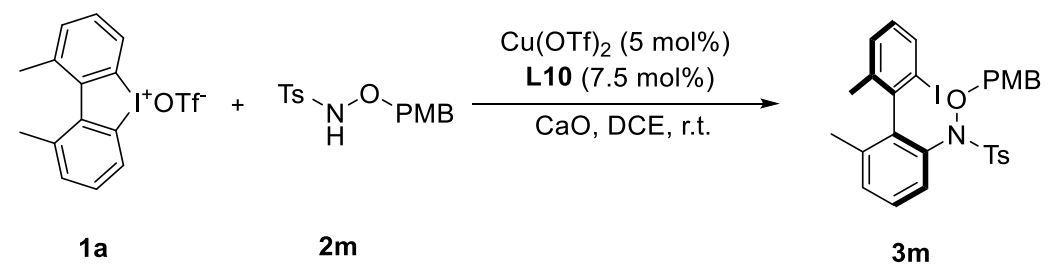

The reaction of $\mathbf{1 a}\left(91.2 \mathrm{mg}, 0.20 \mathrm{mmol}, 1.0\right.$ equiv), $\mathbf{2 m}$ (73.8 mg, $0.24 \mathrm{mmol}, 1.2$ equiv), $\mathrm{Cu}(\mathrm{OTf})_{2}$ (3.6 mg, $5.0 \mathrm{~mol} \%)$, L10 (8.4 mg, $7.5 \mathrm{~mol} \%$ ) and CaO (22.4 mg, $0.40 \mathrm{mmol}, 2.0$ equiv) in DCE (4.0 $\mathrm{mL})$ at room temperature for $12 \mathrm{~h}$ afforded $\mathbf{3 m}(107 \mathrm{mg}, 87 \%, 93 \%$ ee $)$. Rf $=0.4(\mathrm{PE} / \mathrm{EA}=$ 
10:1). $[\alpha]_{D}^{20}=-31.4\left(\mathrm{c}=0.51, \mathrm{CHCl}_{3}\right)$. HPLC conditions: Chiralpak AD-H, isopropanol/hexane $=$ 5:95, flow: $1.0 \mathrm{~mL} / \mathrm{min}, \lambda=254 \mathrm{~nm} .{ }^{1} \mathbf{H}$ NMR $\left(400 \mathrm{MHz}, \mathrm{CDCl}_{3}\right) \delta 7.79(\mathrm{~d}, J=7.6 \mathrm{~Hz}, 1 \mathrm{H}), 7.60-$ $7.53(\mathrm{~m}, 2 \mathrm{H}), 7.34(\mathrm{~d}, J=7.6 \mathrm{~Hz}, 1 \mathrm{H}), 7.28(\mathrm{~d}, J=6.4 \mathrm{~Hz}, 3 \mathrm{H}), 7.23(\mathrm{~d}, J=8.0 \mathrm{~Hz}, 2 \mathrm{H}), 7.10$ (t, $J=$ $7.8 \mathrm{~Hz}, 1 \mathrm{H}), 7.02(\mathrm{t}, J=7.8 \mathrm{~Hz}, 1 \mathrm{H}), 6.87-6.81(\mathrm{~m}, 2 \mathrm{H}), 6.53(\mathrm{~d}, J=8.0 \mathrm{~Hz}, 1 \mathrm{H}), 5.25(\mathrm{~d}, J=9.2 \mathrm{~Hz}$, $1 \mathrm{H}), 4.86(\mathrm{~d}, J=9.2 \mathrm{~Hz}, 1 \mathrm{H}), 3.80(\mathrm{~s}, 3 \mathrm{H}), 2.42(\mathrm{~s}, 3 \mathrm{H}), 2.29$ (s, 3H), $2.03(\mathrm{~s}, 3 \mathrm{H}),{ }^{13} \mathrm{C}$ NMR $(101$ $\left.\mathrm{MHz}, \mathrm{CDCl}_{3}\right) \delta 159.70,144.57,143.00,141.65,140.12,139.81,137.61,136.13,131.58,131.00$, $130.73,130.35,129.93,129.20,129.03,127.84,127.67,124.01,113.67,101.20,78.87,55.23,21.91$, 21.67, 20.22. HRMS (ESI): calcd for $\mathrm{C}_{29} \mathrm{H}_{28} \mathrm{INO}_{4} \mathrm{SNa}(\mathrm{M}+\mathrm{Na})^{+}$636.0681, found 636.0682 .

Compound 3n was prepared following the Procedure A

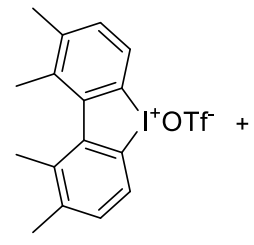

$1 \mathrm{~b}$

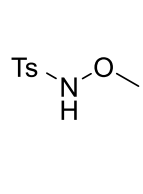

2a

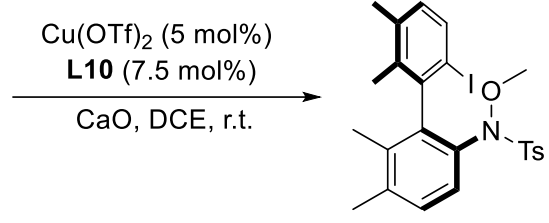

$3 n$

The reaction of $\mathbf{1 b}$ ( $48.4 \mathrm{mg}, 0.10 \mathrm{mmol}, 1.0$ equiv), $\mathbf{2 a}$ ( $24.1 \mathrm{mg}, 0.12 \mathrm{mmol}, 1.2$ equiv), $\mathrm{Cu}(\mathrm{OTf})_{2}(1.8$ mg, $5.0 \mathrm{~mol} \%)$, L10 (4.2 mg, $7.5 \mathrm{~mol} \%$ ) and $\mathrm{CaO}(11.2 \mathrm{mg}, 0.20 \mathrm{mmol}, 2.0$ equiv) in DCE (2.0 mL) at room temperature for $6 \mathrm{~h}$ afforded $3 \mathbf{n}(57 \mathrm{mg},>99 \%, 97 \%$ ee $) . \mathrm{Rf}=0.5(\mathrm{PE} / \mathrm{EA}=10: 1) .[\alpha]_{D}^{20}=$ $+68.9\left(\mathrm{c}=1.05, \mathrm{CHCl}_{3}\right) . \mathrm{HPLC}$ conditions: Chiralpak AD-H, isopropanol/hexane $=5: 95$, flow: 1.0 $\mathrm{mL} / \mathrm{min}, \lambda=254 \mathrm{~nm} .{ }^{1} \mathbf{H}$ NMR $\left(400 \mathrm{MHz}, \mathrm{CDCl}_{3}\right) \delta 7.66(\mathrm{~d}, J=8.0 \mathrm{~Hz}, 1 \mathrm{H}), 7.59(\mathrm{~d}, J=8.4 \mathrm{~Hz}, 2 \mathrm{H})$, $7.28(\mathrm{~d}, J=8.0 \mathrm{~Hz}, 2 \mathrm{H}), 7.04(\mathrm{~d}, J=8.4 \mathrm{~Hz}, 1 \mathrm{H}), 6.91(\mathrm{~d}, J=8.0 \mathrm{~Hz}, 1 \mathrm{H}), 6.52(\mathrm{~d}, J=8.0 \mathrm{~Hz}, 1 \mathrm{H})$, $3.73(\mathrm{~s}, 3 \mathrm{H}), 2.46$ (s, 3H), $2.32(\mathrm{~d}, J=2.4 \mathrm{~Hz}, 6 \mathrm{H}), 2.13$ (s, 3H), 1.90 (s, 3H). ${ }^{13} \mathbf{C}$ NMR (101 MHz, $\left.\mathrm{CDCl}_{3}\right) \delta 144.51,143.90,141.98,138.68,138.28,137.18,136.90,136.00,135.39,132.38,130.57$, 130.03, 129.35, 129.03, 123.30, 97.96, 65.02, 21.67, 20.60, 20.27, 19.29, 16.56. HRMS (ESI): calcd for $\mathrm{C}_{24} \mathrm{H}_{26} \mathrm{INO}_{3} \mathrm{SNa}(\mathrm{M}+\mathrm{Na})^{+} 558.0576$, found 558.0573 .

Compound 30 was prepared following the Procedure A

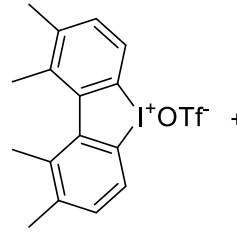

$1 \mathrm{~b}$

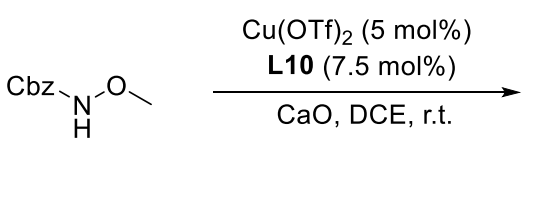

$2 \mathbf{i}$

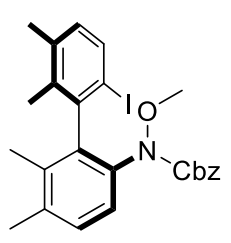

30

The reaction of $\mathbf{1 b}\left(49.2 \mathrm{mg}, 0.10 \mathrm{mmol}, 1.0\right.$ equiv), $\mathbf{2 i}$ ( $24.1 \mathrm{mg}, 0.12 \mathrm{mmol}, 1.2$ equiv), $\mathrm{Cu}(\mathrm{OTf})_{2}(1.8$ $\mathrm{mg}, 5.0 \mathrm{~mol} \%)$, L10 (4.2 mg, $7.5 \mathrm{~mol} \%$ ) and $\mathrm{CaO}(11.2 \mathrm{mg}, 0.20 \mathrm{mmol}, 2.0$ equiv) in DCE (2.0 mL) at room temperature for $6 \mathrm{~h}$ afforded $30(48.9 \mathrm{mg}, 95 \%, 95 \% \mathrm{ee}) . \mathrm{Rf}=0.5(\mathrm{PE} / \mathrm{EA}=10: 1) .[\alpha]_{D}^{20}=$ $+14.6\left(\mathrm{c}=0.98, \mathrm{CHCl}_{3}\right)$. HPLC conditions: Chiralpak AD-H, isopropanol/hexane $=5: 95$, flow: 1.0 $\mathrm{mL} / \mathrm{min}, \lambda=254 \mathrm{~nm} .{ }^{1} \mathbf{H}$ NMR $\left(400 \mathrm{MHz}, \mathrm{CDCl}_{3}\right) \delta 7.63(\mathrm{~d}, J=8.0 \mathrm{~Hz}, 1 \mathrm{H}), 7.35-7.26(\mathrm{~m}, 5 \mathrm{H})$, $7.21(\mathrm{~d}, J=8.0 \mathrm{~Hz}, 1 \mathrm{H}), 7.05(\mathrm{~d}, J=8.0 \mathrm{~Hz}, 1 \mathrm{H}), 6.84(\mathrm{~d}, J=8.0 \mathrm{~Hz}, 1 \mathrm{H}), 5.18(\mathrm{~d}, J=12.4 \mathrm{~Hz}, 1 \mathrm{H})$, $5.14(\mathrm{~d}, J=12.4 \mathrm{~Hz}, 1 \mathrm{H}), 3.45$ (s, 3H), $2.35(\mathrm{~s}, 3 \mathrm{H}), 2.19(\mathrm{~s}, 3 \mathrm{H}), 1.87$ (s, 3H), $1.82(\mathrm{~s}, 3 \mathrm{H}) .{ }^{13} \mathbf{C ~ N M R}$ $\left(101 \mathrm{MHz}, \mathrm{CDCl}_{3}\right) \delta 155.23,142.95,142.04,138.00,137.00,136.63,136.32,136.13,135.69,134.61$, 130.57, 129.24, 128.32, 127.93, 126.23, 98.51, 67.35, 61.51, 20.60, 20.21, 18.03, 16.27. HRMS (ESI): calcd for $\mathrm{C}_{25} \mathrm{H}_{26} \mathrm{INO}_{3} \mathrm{Na}(\mathrm{M}+\mathrm{Na})^{+} 538.0855$, found 538.0848 . 
Compound 3p was prepared following the Procedure A
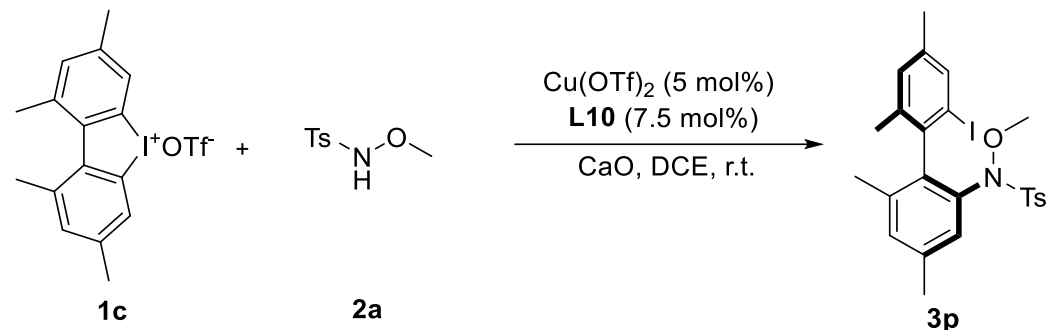

The reaction of $\mathbf{1 c}$ ( $48.4 \mathrm{mg}, 0.10 \mathrm{mmol}, 1.0$ equiv), $\mathbf{2 a}\left(24.1 \mathrm{mg}, 0.12 \mathrm{mmol}, 1.2\right.$ equiv), $\mathrm{Cu}(\mathrm{OTf})_{2}(1.8$ $\mathrm{mg}, 5.0 \mathrm{~mol} \%)$, L10 (4.2 mg, $7.5 \mathrm{~mol} \%)$ and $\mathrm{CaO}(11.2 \mathrm{mg}, 0.20 \mathrm{mmol}, 2.0$ equiv) in DCE (2.0 mL) at room temperature for $6 \mathrm{~h}$ afforded $3 \mathbf{p}(56.8 \mathrm{mg},>99 \%, 94 \% \mathrm{ee}) . \mathrm{Rf}=0.5(\mathrm{PE} / \mathrm{EA}=10: 1) .[\alpha]_{D}^{20}=$ $+0.4\left(\mathrm{c}=1.12, \mathrm{CHCl}_{3}\right)$. HPLC conditions: Chiralpak AD-H, isopropanol/hexane $=5: 95$, flow: 1.0 $\mathrm{mL} / \mathrm{min}, \lambda=254 \mathrm{~nm} .{ }^{1} \mathbf{H}$ NMR $\left(400 \mathrm{MHz}, \mathrm{CDCl}_{3}\right) \delta 7.60(\mathrm{~d}, J=8.4 \mathrm{~Hz}, 3 \mathrm{H}), 7.29(\mathrm{~d}, J=8.0 \mathrm{~Hz}, 2 \mathrm{H})$, $7.10(\mathrm{~d}, J=8.8 \mathrm{~Hz}, 2 \mathrm{H}), 6.36(\mathrm{~s}, 1 \mathrm{H}), 3.77$ (s, 3H), $2.46(\mathrm{~s}, 3 \mathrm{H}), 2.34$ (s, 3H), $2.20(\mathrm{~s}, 3 \mathrm{H}), 2.16(\mathrm{~s}, 3 \mathrm{H})$, 1.97 (s, 3H). ${ }^{13} \mathrm{C}$ NMR $\left(101 \mathrm{MHz}, \mathrm{CDCl}_{3}\right) \delta 144.61,140.22,139.38,139.24,138.83,138.75,137.62$, 137.44, 136.50, 132.16, 131.72, 130.80, 130.17, 128.93, 124.34, 101.42, 65.19, 21.71, 21.67, 20.96, 20.57, 20.11. HRMS (ESI): calcd for $\mathrm{C}_{24} \mathrm{H}_{26} \mathrm{INO}_{3} \mathrm{SNa}(\mathrm{M}+\mathrm{Na})^{+} 558.0576$, found 558.0574.

Compound 3q was prepared following the Procedure A
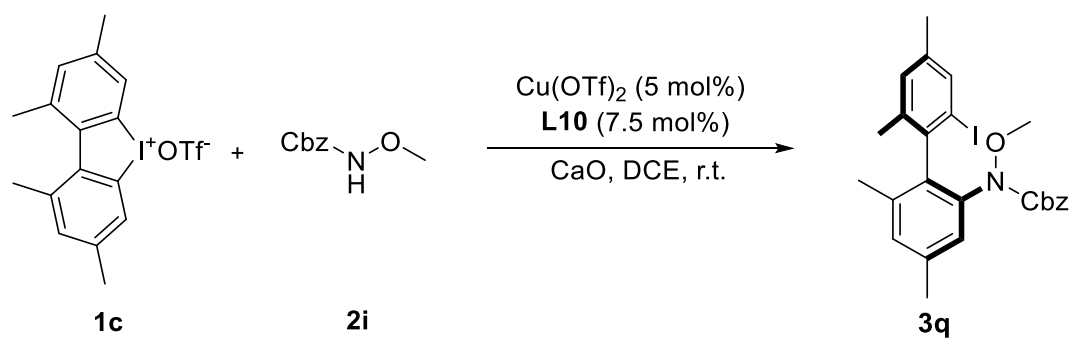

The reaction of $1 \mathbf{c}$ (49.2 mg, $0.10 \mathrm{mmol}, 1.0$ equiv), $2 \mathbf{i}\left(24.1 \mathrm{mg}, 0.12 \mathrm{mmol}, 1.2\right.$ equiv), $\mathrm{Cu}(\mathrm{OTf})_{2}(1.8$ $\mathrm{mg}, 5.0 \mathrm{~mol} \%), \mathbf{L 1 0}(4.2 \mathrm{mg}, 7.5 \mathrm{~mol} \%)$ and $\mathrm{CaO}(11.2 \mathrm{mg}, 0.20 \mathrm{mmol}, 2.0$ equiv) in DCE (2.0 mL) at room temperature for $6 \mathrm{~h}$ afforded $3 \mathbf{q}(38.6 \mathrm{mg}, 75 \%, 89 \% \mathrm{ee}) . \mathrm{Rf}=0.5(\mathrm{PE} / \mathrm{EA}=10: 1) .[\alpha]_{D}^{20}=$ $+32.3\left(\mathrm{c}=0.97, \mathrm{CHCl}_{3}\right)$. HPLC conditions: Chiralpak AD-H, isopropanol/hexane $=5: 95$, flow: 1.0 $\mathrm{mL} / \mathrm{min}, \lambda=254 \mathrm{~nm} .{ }^{1} \mathbf{H}$ NMR $\left(400 \mathrm{MHz}, \mathrm{CDCl}_{3}\right) \delta 7.58(\mathrm{~s}, 1 \mathrm{H}), 7.37-7.27(\mathrm{~m}, 5 \mathrm{H}), 7.14(\mathrm{~s}, 1 \mathrm{H})$, $6.99(\mathrm{~s}, 1 \mathrm{H}), 6.94(\mathrm{~s}, 1 \mathrm{H}), 5.18(\mathrm{~s}, 2 \mathrm{H}), 3.50(\mathrm{~s}, 3 \mathrm{H}), 2.37$ (s, 3H), 2.29 (s, 3H), 1.96 (s, 3H), $1.91(\mathrm{~s}$, 3H). ${ }^{13}$ C NMR $\left(101 \mathrm{MHz}, \mathrm{CDCl}_{3}\right) \delta 155.22,139.23,138.99,138.72,137.86,137.70,137.67,136.83$, $136.74,136.10,131.49,130.63,128.32,127.96,127.92,127.20,101.89,67.38,61.83,21.12,21.00$, 20.55, 19.76. HRMS (ESI): calcd for $\mathrm{C}_{25} \mathrm{H}_{26} \mathrm{INO}_{3} \mathrm{Na}(\mathrm{M}+\mathrm{Na})^{+} 538.0855$, found 538.0851.

Compound 3r was prepared following the Procedure A 

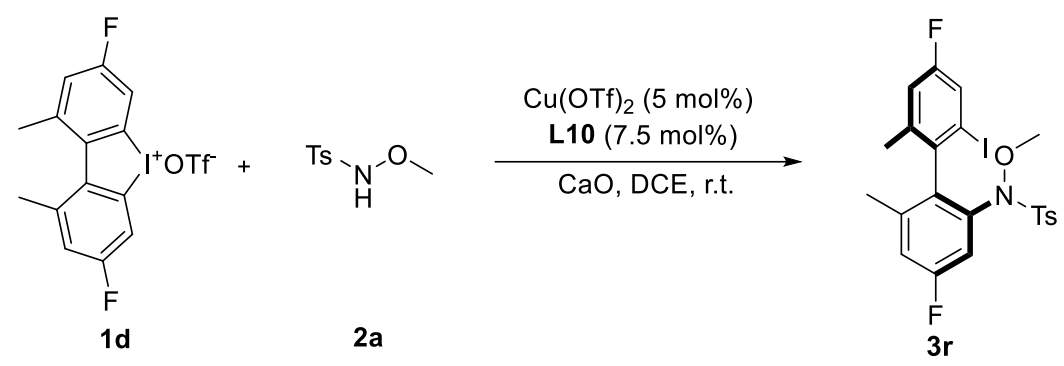

The reaction of $\mathbf{1 d}\left(49.2 \mathrm{mg}, 0.10 \mathrm{mmol}, 1.0\right.$ equiv), $\mathbf{2 a}\left(24.1 \mathrm{mg}, 0.12 \mathrm{mmol}, 1.2\right.$ equiv), $\mathrm{Cu}(\mathrm{OTf})_{2}(1.8$ $\mathrm{mg}, 5.0 \mathrm{~mol} \%), \mathbf{L 1 0}(4.2 \mathrm{mg}, 7.5 \mathrm{~mol} \%)$ and $\mathrm{CaO}(11.2 \mathrm{mg}, 0.20 \mathrm{mmol}, 2.0$ equiv) in DCE (2.0 mL) at room temperature for $6 \mathrm{~h}$ afforded $3 \mathbf{r}(46 \mathrm{mg}, 85 \%, 96 \%$ ee $) . \operatorname{Rf}=0.5(\mathrm{PE} / \mathrm{EA}=10: 1) . \quad[\alpha]_{D}^{20}=+91.5$ (c $=0.96, \mathrm{CHCl}_{3}$ ). HPLC conditions: Chiralpak AD-H, isopropanol/hexane $=5: 95$, flow: $1.0 \mathrm{~mL} / \mathrm{min}, \lambda$ $=254 \mathrm{~nm} .{ }^{1} \mathbf{H}$ NMR $\left(400 \mathrm{MHz}, \mathrm{CDCl}_{3}\right) \delta 7.58(\mathrm{~d}, J=8.4 \mathrm{~Hz}, 2 \mathrm{H}), 7.51(\mathrm{dd}, J=7.8,2.6 \mathrm{~Hz}, 1 \mathrm{H}), 7.32$ $(\mathrm{d}, J=8.0 \mathrm{~Hz}, 2 \mathrm{H}), 7.07(\mathrm{dd}, J=9.2,2.4 \mathrm{~Hz}, 1 \mathrm{H}), 7.01(\mathrm{dd}, J=8.8,2.4 \mathrm{~Hz}, 1 \mathrm{H}), 6.25(\mathrm{dd}, J=9.6,2.4$ $\mathrm{Hz}, 1 \mathrm{H}), 3.83$ (s, 3H), 2.47 (s, 3H), 2.23 (s, 3H), 1.99 (s, 3H). ${ }^{19}$ F NMR $\left(376 \mathrm{MHz} \mathrm{CDCl}_{3}\right) \delta-112.49$, -112.51, -112.54, -114.08, -114.10, -114.10, -114.13. $\left.{ }^{13} \mathbf{C ~ N M R ~ ( 1 0 1 ~ M H z , ~} \mathrm{CDCl}_{3}\right) \delta$ 162.66, 160.18, 145.24, $141.63(\mathrm{~d}, J=7.9 \mathrm{~Hz}), 141.33(\mathrm{~d}, J=9.1 \mathrm{~Hz}), 139.81(\mathrm{~d}, J=8.8 \mathrm{~Hz}), 137.91(\mathrm{~d}, J=3.6 \mathrm{~Hz})$, 136.89 (d, $J=3.4 \mathrm{~Hz}), 130.89,130.20,129.24,123.24$ (d, $J=23.5 \mathrm{~Hz}), 117.77$ (d, $J=21.2 \mathrm{~Hz}), 116.89$ $(\mathrm{d}, J=20.6 \mathrm{~Hz}), 110.80(\mathrm{~d}, J=23.5 \mathrm{~Hz}), 100.52(\mathrm{~d}, J=7.9 \mathrm{~Hz}), 65.42,22.13(\mathrm{~d}, J=1.5 \mathrm{~Hz}), 21.74$, $20.35(\mathrm{~d}, J=1.8 \mathrm{~Hz})$. HRMS (ESI): calcd for $\mathrm{C}_{22} \mathrm{H}_{20} \mathrm{~F}_{2} \mathrm{INO}_{3} \mathrm{SNa}(\mathrm{M}+\mathrm{Na})^{+}$566.0074, found 566.0078.

Compound 3s was prepared following the Procedure A<smiles></smiles>

$1 e$

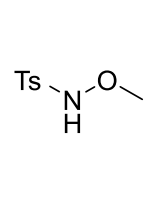

$2 a$

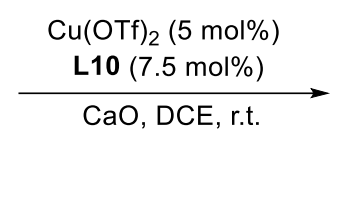

The reaction of $1 \mathbf{e}\left(26.4 \mathrm{mg}, 0.05 \mathrm{mmol}, 1.0\right.$ equiv), $2 \mathbf{a}\left(12 \mathrm{mg}, 0.06 \mathrm{mmol}, 1.2\right.$ equiv), $\mathrm{Cu}(\mathrm{OTf})_{2}(0.9$ $\mathrm{mg}, 5.0 \mathrm{~mol} \%), \mathbf{L 1 0}(2.4 \mathrm{mg}, 7.5 \mathrm{~mol} \%)$ and $\mathrm{CaO}(5.6 \mathrm{mg}, 0.10 \% \mathrm{mmol}, 2.0$ equiv) in DCE (1.0 mL) at room temperature for $12 \mathrm{~h}$ afforded $3 \mathrm{~s}(21.1 \mathrm{mg}, 73 \%,>99 \% \mathrm{ee}) . \mathrm{Rf}=0.3(\mathrm{PE} / \mathrm{EA}=10: 1) .[\alpha]_{D}^{20}=$ -24.2 $\left(\mathrm{c}=0.38, \mathrm{CHCl}_{3}\right)$. HPLC conditions: Chiralpak AD-H, isopropanol/hexane = 10:90, flow: 1.0 $\mathrm{mL} / \mathrm{min}, \lambda=254 \mathrm{~nm} .{ }^{1} \mathbf{H}$ NMR $\left(400 \mathrm{MHz}, \mathrm{CDCl}_{3}\right) \delta 8.04(\mathrm{~d}, J=8.8 \mathrm{~Hz}, 1 \mathrm{H}), 7.92(\mathrm{dd}, J=8.2,2.8 \mathrm{~Hz}$, 2H), $7.84(\mathrm{~d}, J=8.8 \mathrm{~Hz}, 1 \mathrm{H}), 7.70(\mathrm{~d}, J=8.4 \mathrm{~Hz}, 1 \mathrm{H}), 7.53(\mathrm{tdd}, J=8.0,6.8,1.2 \mathrm{~Hz}, 2 \mathrm{H}), 7.43-7.29$ (m, 5H), $7.21(\mathrm{dd}, J=8.4,3.2 \mathrm{~Hz}, 3 \mathrm{H}), 7.03(\mathrm{~d}, J=8.8 \mathrm{~Hz}, 1 \mathrm{H}), 3.55(\mathrm{~s}, 3 \mathrm{H}), 2.44(\mathrm{~s}, 3 \mathrm{H}) .{ }^{13} \mathbf{C}$ NMR $\left(101 \mathrm{MHz}, \mathrm{CDCl}_{3}\right) \delta 144.71,139.89,139.30,137.17,135.54,134.27,133.43,132.62,132.37,132.15$, $129.94,129.54,129.17,129.14,128.70,128.02$, 127.61, 127.20, 127.17, 126.98, 126.41, 126.23, 123.23, 100.64, 65.13, 21.70. HRMS (ESI): calcd for $\mathrm{C}_{28} \mathrm{H}_{22} \mathrm{INO}_{3} \mathrm{SNa}(\mathrm{M}+\mathrm{Na})^{+} 602.0263$, found 602.0273 .

Compound $\mathbf{3 t}$ was prepared following the Procedure A 


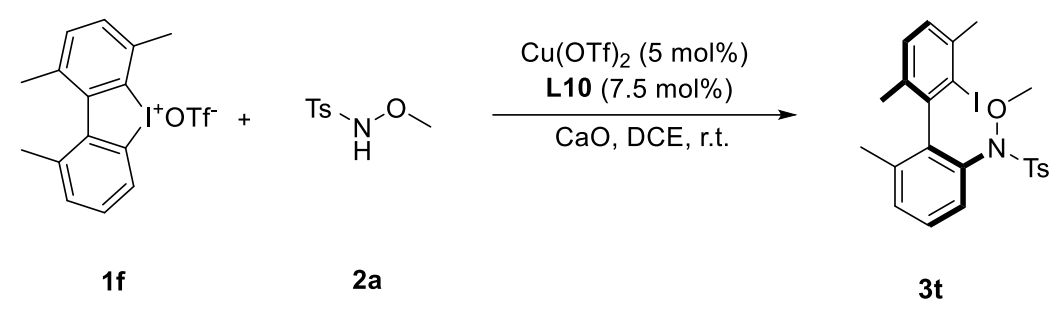

The reaction of $\mathbf{1 f}\left(47.0 \mathrm{mg}, 0.10 \mathrm{mmol}, 1.0\right.$ equiv), $\mathbf{2 a}\left(24.1 \mathrm{mg}, 0.12 \mathrm{mmol}, 1.2\right.$ equiv), $\mathrm{Cu}(\mathrm{OTf})_{2}(1.8$ $\mathrm{mg}, 5.0 \mathrm{~mol} \%), \mathbf{L 1 0}(4.2 \mathrm{mg}, 7.5 \mathrm{~mol} \%)$ and $\mathrm{CaO}(11.2 \mathrm{mg}, 0.20 \mathrm{mmol}, 2.0$ equiv) in DCE (2.0 mL) at room temperature for $6 \mathrm{~h}$ afforded $3 \mathrm{t}(48.2 \mathrm{mg}, 93 \%, 95 \% \mathrm{ee}) . \mathrm{Rf}=0.5(\mathrm{PE} / \mathrm{EA}=10: 1) . \quad[\alpha]_{D}^{20}=+0.7$ ( $\left.\mathrm{c}=1.05, \mathrm{CHCl}_{3}\right)$. HPLC conditions: Chiralpak AD-H, isopropanol/hexane $=5: 95$, flow: $1.0 \mathrm{~mL} / \mathrm{min}, \lambda$ $=254 \mathrm{~nm} .{ }^{1} \mathbf{H} \mathbf{N M R}\left(400 \mathrm{MHz}, \mathrm{CDCl}_{3}\right) \delta 7.61(\mathrm{~d}, J=8.0 \mathrm{~Hz}, 2 \mathrm{H}), 7.28(\mathrm{~d}, J=7.6 \mathrm{~Hz}, 3 \mathrm{H}), 7.22-$ $7.17(\mathrm{~m}, 2 \mathrm{H}), 7.15(\mathrm{t}, J=8.0 \mathrm{~Hz}, 1 \mathrm{H}), 6.63(\mathrm{~d}, J=7.6 \mathrm{~Hz}, 1 \mathrm{H}), 3.70(\mathrm{~s}, 3 \mathrm{H}), 2.49$ (s, 3H), $2.46(\mathrm{~s}, 3 \mathrm{H})$, $2.21(\mathrm{~s}, 3 \mathrm{H}), 2.01(\mathrm{~s}, 3 \mathrm{H}) .{ }^{13} \mathbf{C} \mathbf{N M R}\left(101 \mathrm{MHz}, \mathrm{CDCl}_{3}\right) \delta 144.64,144.24,142.16,139.35,138.92$, 137.72, 136.57, 132.20, 130.90, 130.06, 129.58, 129.08, 128.67, 127.78, 123.74, 107.86, 65.12, 29.55, 21.69, 21.41, 20.14. HRMS (ESI): calcd for $\mathrm{C}_{23} \mathrm{H}_{24} \mathrm{INO}_{3} \mathrm{SNa}(\mathrm{M}+\mathrm{Na})^{+} 544.0419$, found 544.0421.

Synthesis of compound $\mathbf{4 a}$
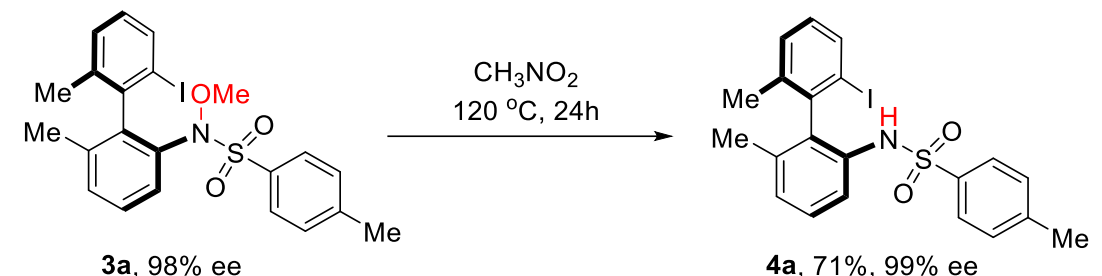

In a $10 \mathrm{~mL}$ schlenk tube capped with PTFE screw cap, 3a (101 mg, $0.20 \mathrm{mmol}, 98 \%$ ee) was dissolved in $\mathrm{CH}_{3} \mathrm{NO}_{2}(1.0 \mathrm{~mL})$ under nitrogen. The mixture was heated to $120{ }^{\circ} \mathrm{C}$ and stirred for $24 \mathrm{~h}$. After complete consumption of starting material, the reaction was cooled to room temperature, the solvent was removed and the residue was purified by column chromatography on silica gel (hexanes/ethyl acetate $=20: 1)$ to afford the desired product $4 \mathbf{a}(67.7 \mathrm{mg}, 71 \%, 99 \% \mathrm{ee})$. Rf $=0.3(\mathrm{PE} / \mathrm{EA}=$ 10:1). $[\alpha]_{D}^{20}=+31.6\left(\mathrm{c}=0.98, \mathrm{CHCl}_{3}\right)$. HPLC conditions: Chiralpak ID-H, isopropanol/hexane = 5:95, flow: $1.0 \mathrm{~mL} / \mathrm{min}, \lambda=254 \mathrm{~nm} .{ }^{1} \mathbf{H} \mathbf{N M R}\left(400 \mathrm{MHz}, \mathrm{CDCl}_{3}\right) \delta 7.81(\mathrm{~d}, J=8.0 \mathrm{~Hz}, 1 \mathrm{H}), 7.77-$ $7.71(\mathrm{~m}, 2 \mathrm{H}), 7.51(\mathrm{~d}, J=8.0 \mathrm{~Hz}, 1 \mathrm{H}), 7.28(\mathrm{~d}, J=7.6 \mathrm{~Hz}, 1 \mathrm{H}), 7.23(\mathrm{t}, J=8.6 \mathrm{~Hz}, 3 \mathrm{H}), 7.04(\mathrm{t}, J=7.8$ $\mathrm{Hz}, 1 \mathrm{H}), 6.98$ (d, $J=7.6 \mathrm{~Hz}, 1 \mathrm{H}), 5.98(\mathrm{~s}, 1 \mathrm{H}), 2.38(\mathrm{~s}, 3 \mathrm{H}), 1.83$ (s, 3H), $1.76(\mathrm{~s}, 3 \mathrm{H}) .{ }^{13} \mathbf{C} \mathbf{N M R}(101$ $\left.\mathrm{MHz}, \mathrm{CDCl}_{3}\right) \delta 143.92,139.51,139.07,137.46,136.73,136.60,133.73,132.53,130.69,130.40$, 129.62, 128.67, 127.55, 125.44, 114.96, 101.38, 21.48, 20.77, 19.70. HRMS (ESI): calcd for $\mathrm{C}_{21} \mathrm{H}_{20} \mathrm{INO}_{2} \mathrm{SNa}(\mathrm{M}+\mathrm{Na})^{+} 500.0157$, found 500.0156 .

Synthesis of compound $\mathbf{5 j}$

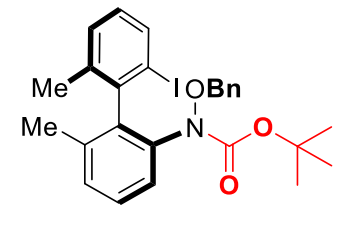

3j, 95\%ee

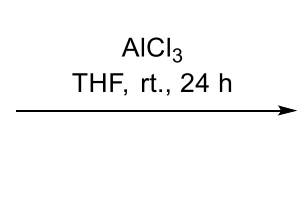

$\mathbf{5 j}, 78 \%, 96 \%$ ee

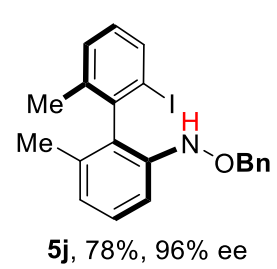

To a $25 \mathrm{~mL}$ Schleck tube containing $3 \mathbf{j}$ (106 mg, $0.20 \mathrm{mmol}$, 1.0equiv, 95\% ee) and THF (1.0 mL) was added anhydrous $\mathrm{AlCl}_{3}$ ( $80 \mathrm{mg}, 0.60 \mathrm{mmol}, 3.0$ equiv) as one portion under nitrogen, and stirred for 24 
$\mathrm{h}$ at room temperature. After complete consumption of starting material, the solution was quenched with $4 \mathrm{M} \mathrm{HCl}$ solution $(2 \mathrm{~mL})$ and water $(10 \mathrm{~mL})$, extracted with ethyl acetate $(10 \mathrm{~mL} \times 2)$, dried over anhydrous sodium sulfate and purified by flash column chromatography on silica gel (hexanes/ethyl acetate $=50: 1)$ to afford the desired product $\mathbf{5 j}(66.4 \mathrm{mg}, 78 \%, 96 \%$ ee $) . \mathrm{Rf}=0.7(\mathrm{PE} / \mathrm{EA}=$ 20:1). $[\alpha]_{D}^{20}=+39.8\left(\mathrm{c}=1.23, \mathrm{CHCl}_{3}\right)$. HPLC conditions: Chiralpak OD-H, isopropanol/hexane = 1:99, flow: $1.0 \mathrm{~mL} / \mathrm{min}, \lambda=254 \mathrm{~nm} .{ }^{1} \mathbf{H} \mathbf{N M R}\left(400 \mathrm{MHz}, \mathrm{CDCl}_{3}\right) \delta 7.82(\mathrm{t}, J=7.6 \mathrm{~Hz}, 1 \mathrm{H}), 7.43-$ $7.26(\mathrm{~m}, 7 \mathrm{H}), 7.15(\mathrm{t}, J=9.2 \mathrm{~Hz}, 1 \mathrm{H}), 7.04-6.90(\mathrm{~m}, 2 \mathrm{H}), 6.52(\mathrm{~d}, J=10.8 \mathrm{~Hz}, 1 \mathrm{H}), 4.80(\mathrm{qd}, J=$ 11.1, 8.4 Hz, 2H), $2.05(\mathrm{~d}, J=9.2 \mathrm{~Hz}, 3 \mathrm{H}), 1.92(\mathrm{~d}, J=9.6 \mathrm{~Hz}, 3 \mathrm{H}) .{ }^{13} \mathbf{C} \mathbf{N M R}\left(101 \mathrm{MHz}, \mathrm{CDCl}_{3}\right) \delta$ $144.75,140.90,138.90,137.13,136.50,135.73,130.30,129.56,129.19,128.92,128.68,128.38$, 128.14, 123.26, 112.13, 101.40, 77.35, 21.17, 19.32. HRMS (ESI): calcd for $\mathrm{C}_{21} \mathrm{H}_{20} \mathrm{INONa}(\mathrm{M}+\mathrm{Na})^{+}$ 452.0487, found 452.0489.

Synthesis of compound $\mathbf{6} \mathbf{j}$ and $\mathbf{7} \mathbf{j}$<smiles>Cc1cccc(OCc2ccccc2)c1-c1c(C)cccc1OC(C)C</smiles>

from $1.0 \mathrm{mmol}$ scale reaction

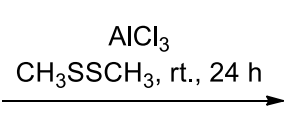

$91 \%, 95 \%$ ee

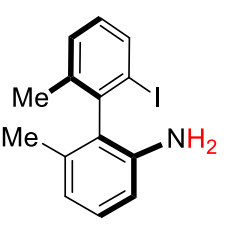

6j, $32 \%, 95 \%$ ee

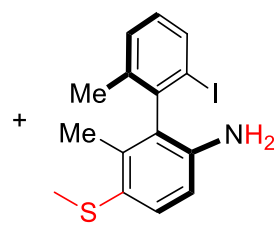

$7 j, 34 \%, 95 \%$ ee

To a $25 \mathrm{~mL}$ Schleck tube containing $3 \mathbf{j}$ (481.3 mg, $0.91 \mathrm{mmol}, 1.0$ equiv, 95\% ee) and dimethyl disulfide $(5.0 \mathrm{~mL})$ was added anhydrous $\mathrm{AlCl}_{3}(364 \mathrm{mg}, 2.73 \mathrm{mmol}, 3.0$ equiv) as one portion under nitrogen, and stirred for $24 \mathrm{~h}$ at room temperature. After complete consumption of starting material, the solution was quenched with $4 \mathrm{M} \mathrm{HCl}$ solution $(4 \mathrm{~mL})$ and water $(10 \mathrm{~mL})$, extracted with ethyl acetate (10 mL x 2), dried over anhydrous sodium sulfate and purified by flash column chromatography on silica gel (hexanes/ethyl acetate $=50: 1)$ to afford product $\mathbf{6 j}$ (92.6 mg, 32\%, 95\% ee) and $7 \mathbf{j}$ ( $114.2 \mathrm{mg}$, $34 \%, 95 \%$ ee).

6j: $\mathrm{Rf}=0.5(\mathrm{PE} / \mathrm{EA}=10: 1) .[\alpha]_{D}^{20}=+34.1\left(\mathrm{c}=0.74, \mathrm{CHCl}_{3}\right)$. HPLC conditions: Chiralpak AD-H, isopropanol/hexane = 1:99, flow: $1.0 \mathrm{~mL} / \mathrm{min}, \lambda=254 \mathrm{~nm} .{ }^{1} \mathbf{H} \mathbf{N M R}\left(400 \mathrm{MHz}, \mathrm{CDCl}_{3}\right) \delta 7.83(\mathrm{~d}, J=$ $8.0 \mathrm{~Hz}, 1 \mathrm{H}), 7.30(\mathrm{~d}, J=7.6 \mathrm{~Hz}, 1 \mathrm{H}), 7.13(\mathrm{t}, J=7.6 \mathrm{~Hz}, 1 \mathrm{H}), 6.98(\mathrm{t}, J=7.6 \mathrm{~Hz}, 1 \mathrm{H}), 6.73(\mathrm{~d}, J=7.6$ $\mathrm{Hz}, 1 \mathrm{H}), 6.65(\mathrm{~d}, J=8.0 \mathrm{~Hz}, 1 \mathrm{H}), 3.29(\mathrm{~s}, 2 \mathrm{H}), 2.09$ (s, 3H), 1.89 (s, 3H). ${ }^{13} \mathbf{C} \mathbf{N M R}\left(101 \mathrm{MHz}, \mathrm{CDCl}_{3}\right)$ $\delta 142.87,142.04,139.04,137.14,136.22,130.31,129.65,129.35,128.53,120.01,112.92,102.08$, 21.13, 19.71. HRMS (ESI): calcd for $\mathrm{C}_{14} \mathrm{H}_{14} \mathrm{INNa}(\mathrm{M}+\mathrm{Na})^{+} 346.0069$, found 346.0063 .

7j: $\mathrm{Rf}=0.4(\mathrm{PE} / \mathrm{EA}=10: 1) .[\alpha]_{D}^{20}=-14.1\left(\mathrm{c}=1.40, \mathrm{CHCl}_{3}\right)$. HPLC conditions: Chiralpak AD-H, isopropanol/hexane $=2: 98$, flow: $1.0 \mathrm{~mL} / \mathrm{min}, \lambda=254 \mathrm{~nm} .{ }^{1} \mathbf{H} \mathbf{N M R}\left(400 \mathrm{MHz}, \mathrm{CDCl}_{3}\right) \delta 7.82(\mathrm{~d}, J=$ $7.6 \mathrm{~Hz}, 1 \mathrm{H}), 7.30(\mathrm{~d}, J=6.8 \mathrm{~Hz}, 1 \mathrm{H}), 7.24(\mathrm{~d}, J=8.4 \mathrm{~Hz}, 1 \mathrm{H}), 6.98(\mathrm{t}, J=7.6 \mathrm{~Hz}, 1 \mathrm{H}), 6.66(\mathrm{~d}, J=8.4$ Hz, 1H), 3.67 - 2.61 (broad, 2H), 2.40 (s, 3H), 2.07 (s, 3H), 1.99 (s, 3H). $\left.{ }^{13} \mathbf{C ~ N M R ~ ( 1 0 1 ~ M H z , ~} \mathrm{CDCl}_{3}\right)$ $\delta 142.09,141.70,139.00,137.23,136.52,130.41,130.37,130.12,129.45,125.49,113.51,101.99$, 21.16, 18.05, 17.00. HRMS (ESI): calcd for $\mathrm{C}_{15} \mathrm{H}_{17} \mathrm{INS}(\mathrm{M}+\mathrm{H})^{+} 370.0126$, found 370.0131 .

\section{References}

1. Zhao, K.; Duan, L.; Xu, S.; Jiang, L.; Fu, Y.; Gu, Z. Chem 2018, 4, 599.

2. Reddy, L, R.; Gupta, A, P.; Liu, Y. J. Org. Chem. 2011, 76, 3409.

3. Zuo, Z.; Cong, H.; Li, W.; Choi, J.; Fu, G, C.; Macmillan, W, C. J. Am. Chem. Soc. 2016, 138, 1832. 


\section{Copies of NMR Spectroscopies}
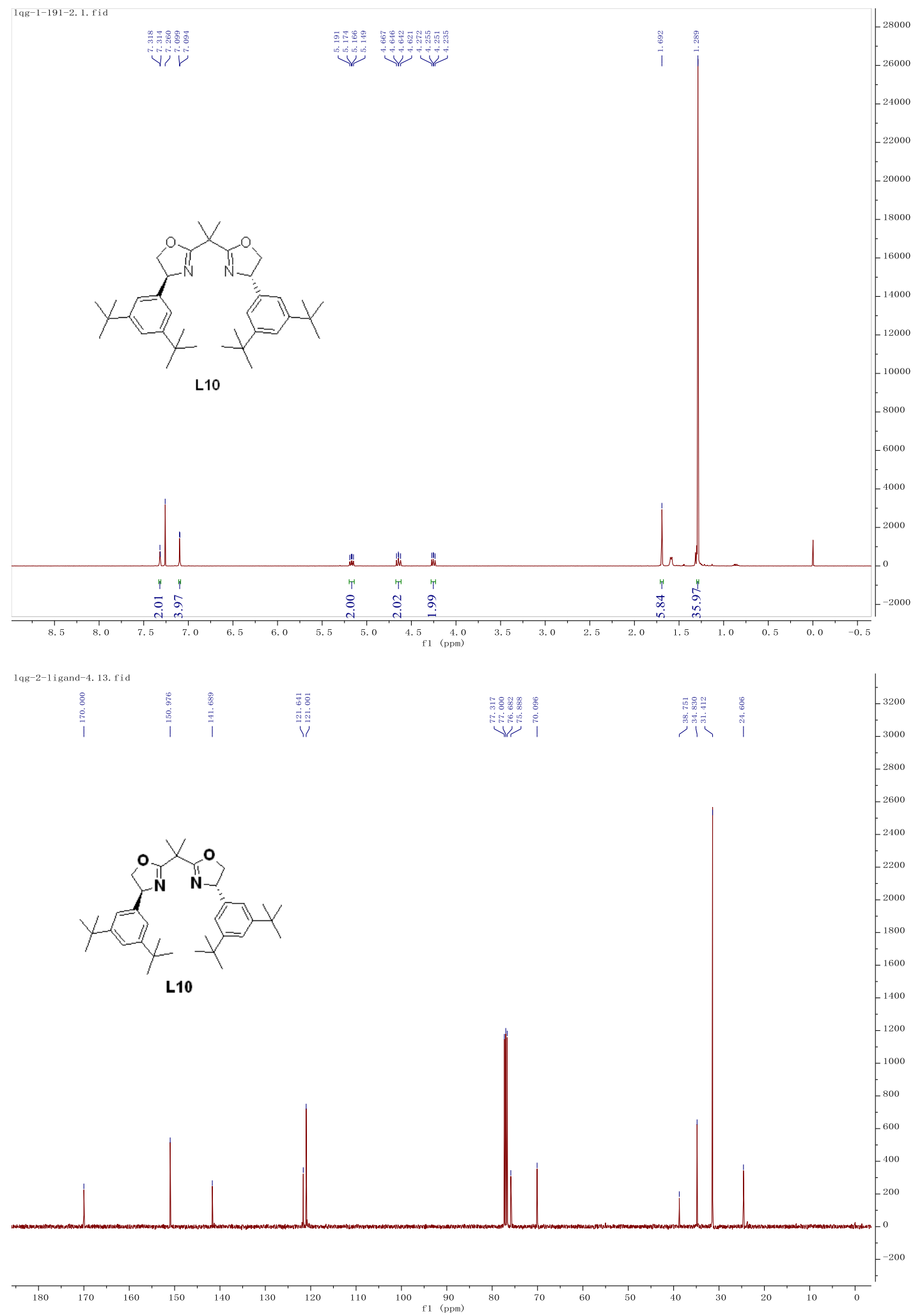

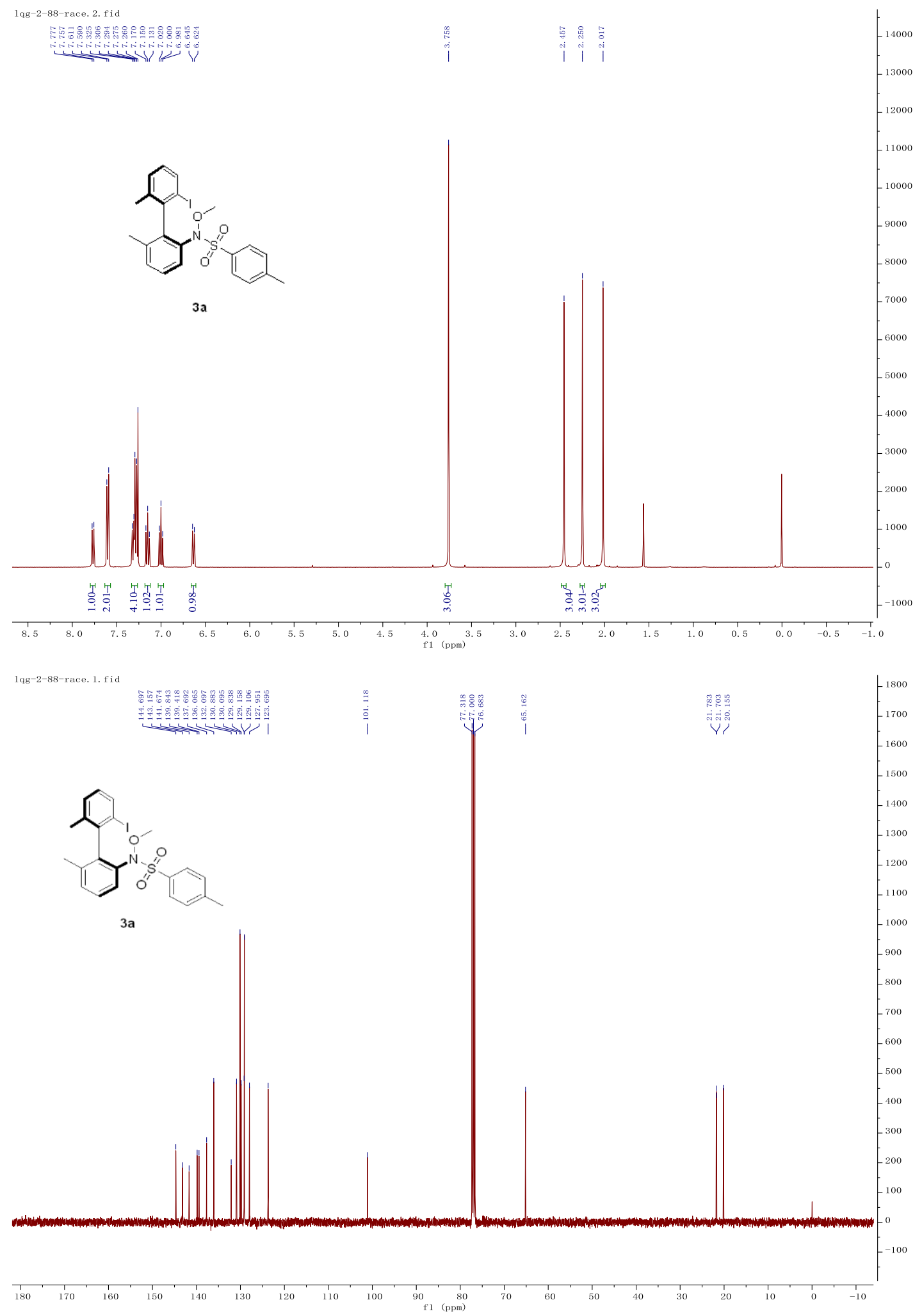

S15 


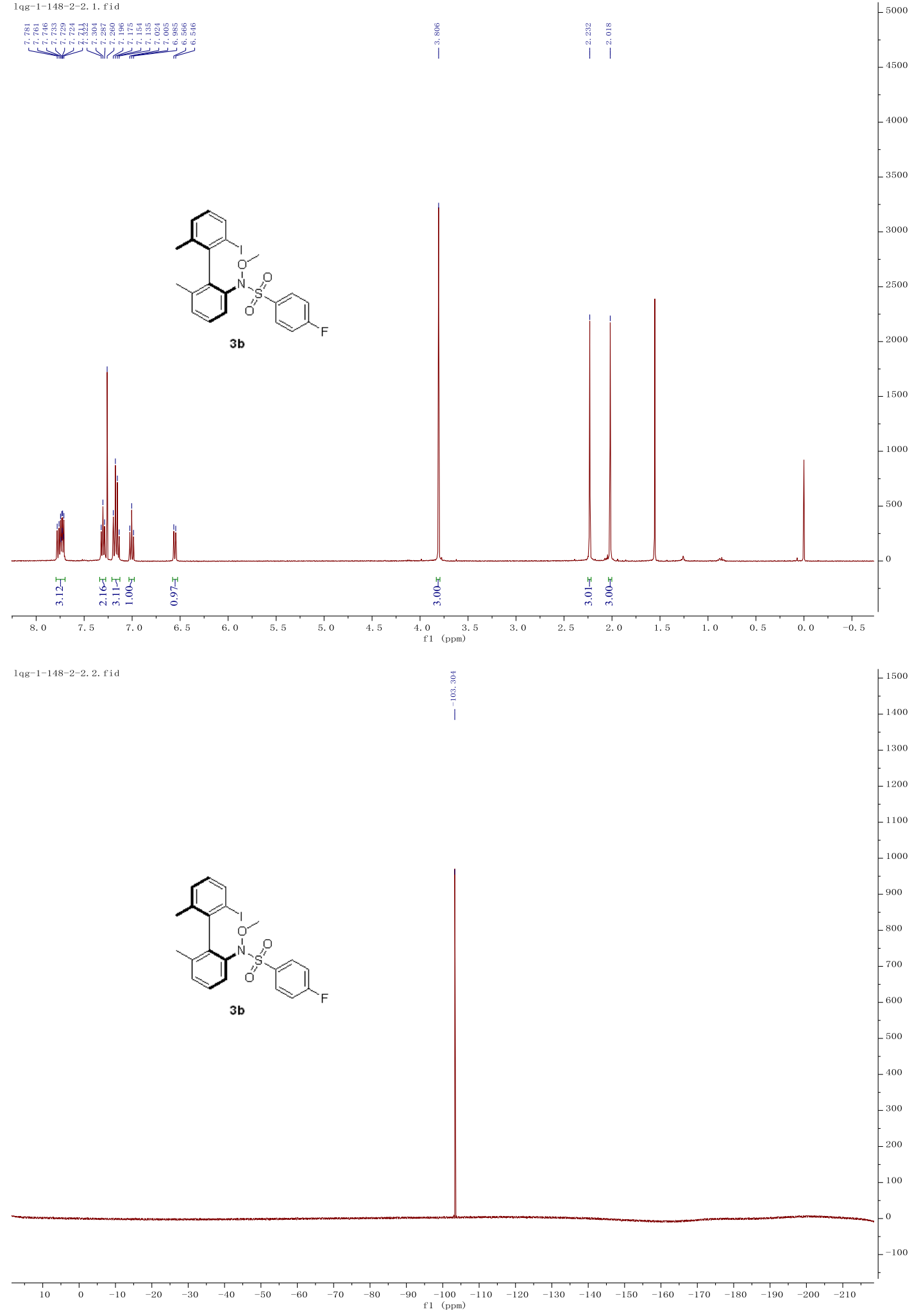




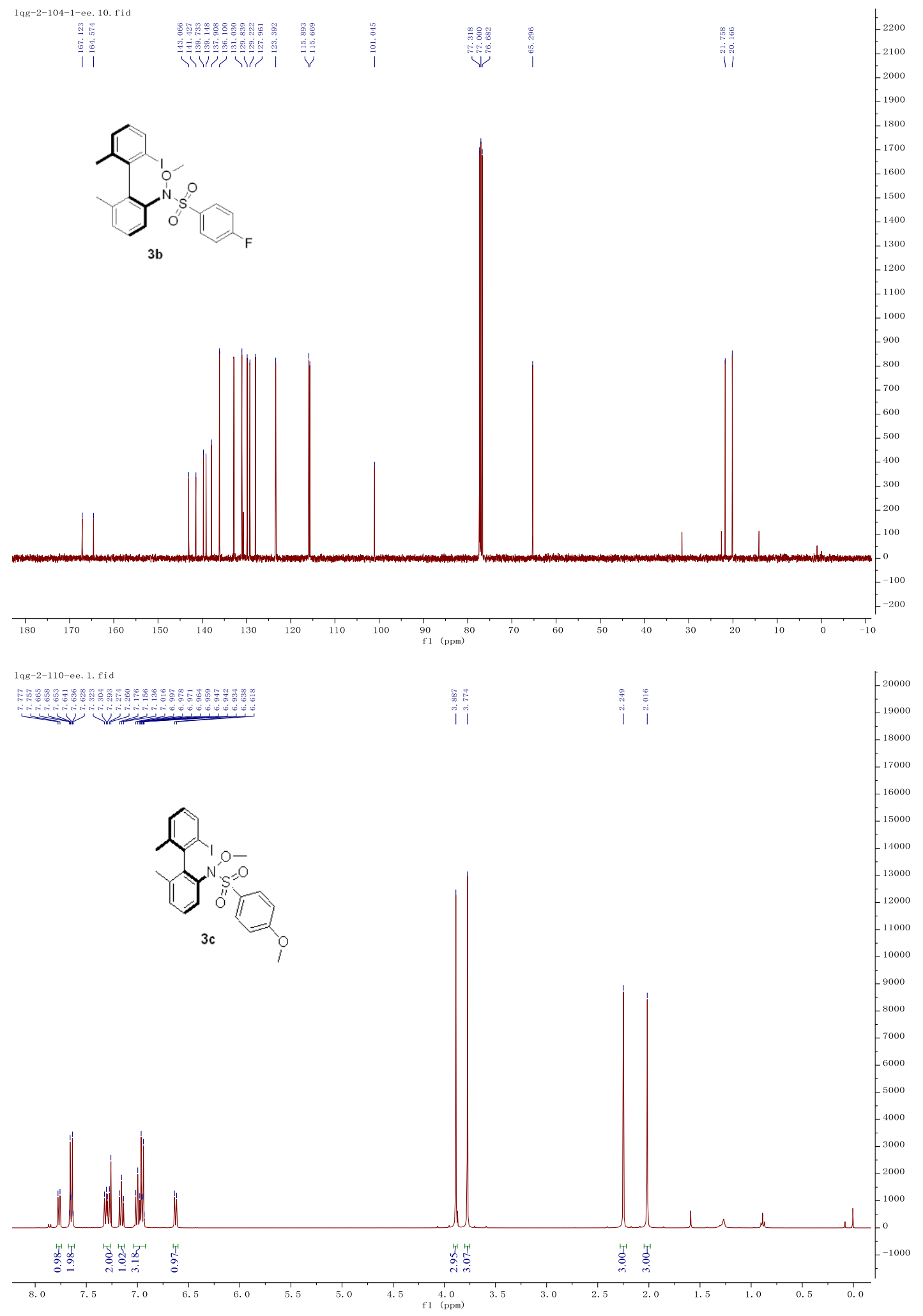




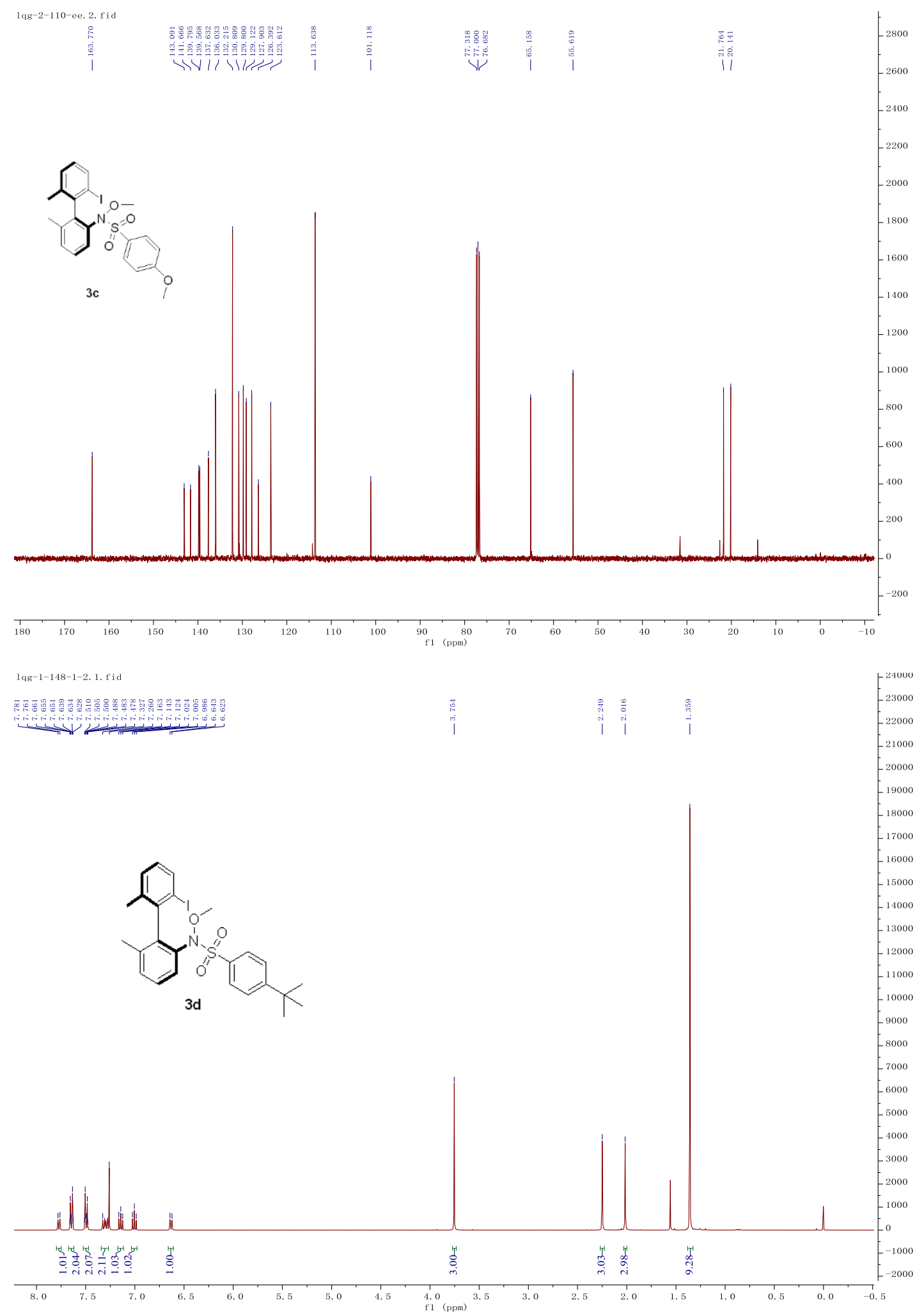




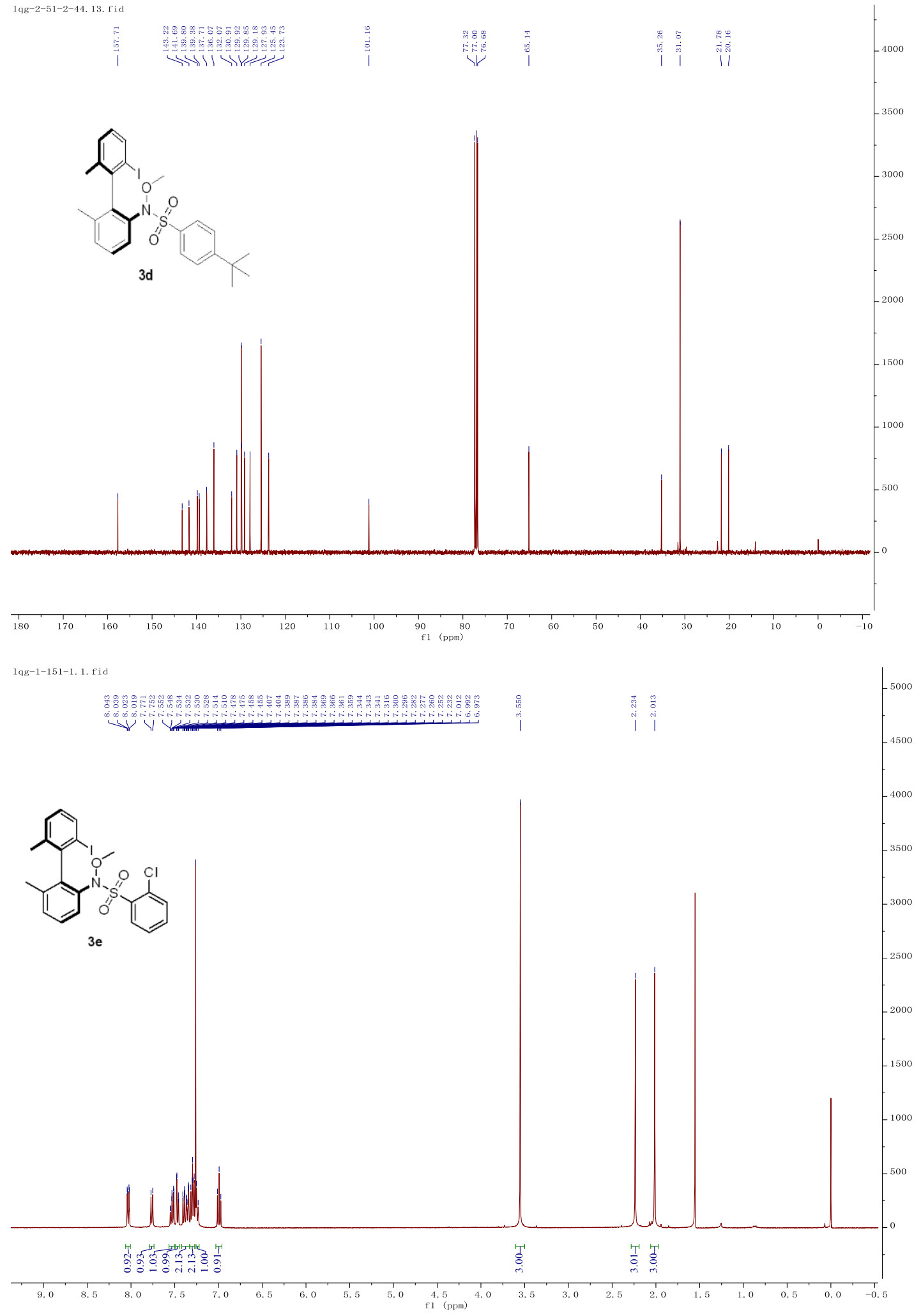




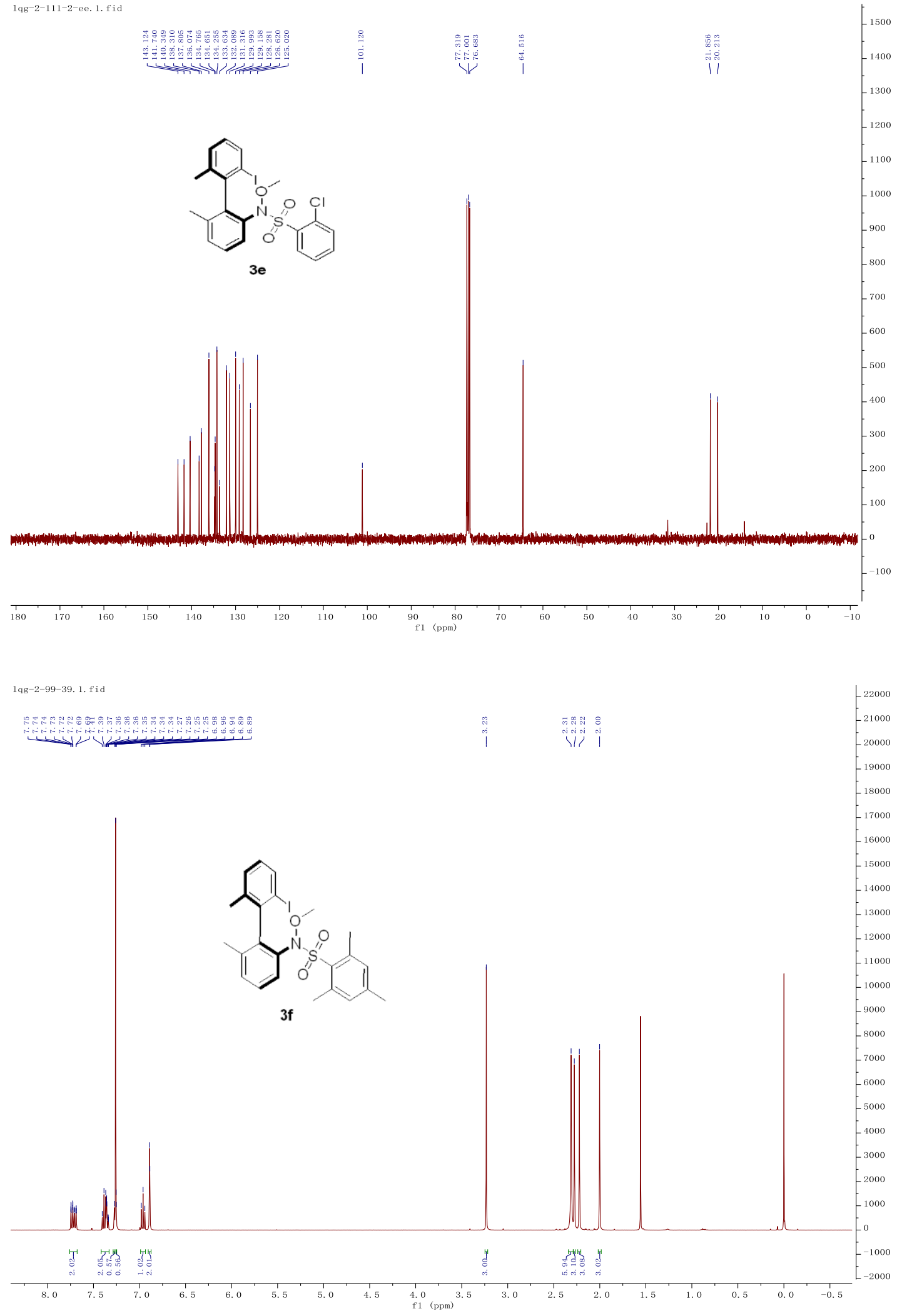




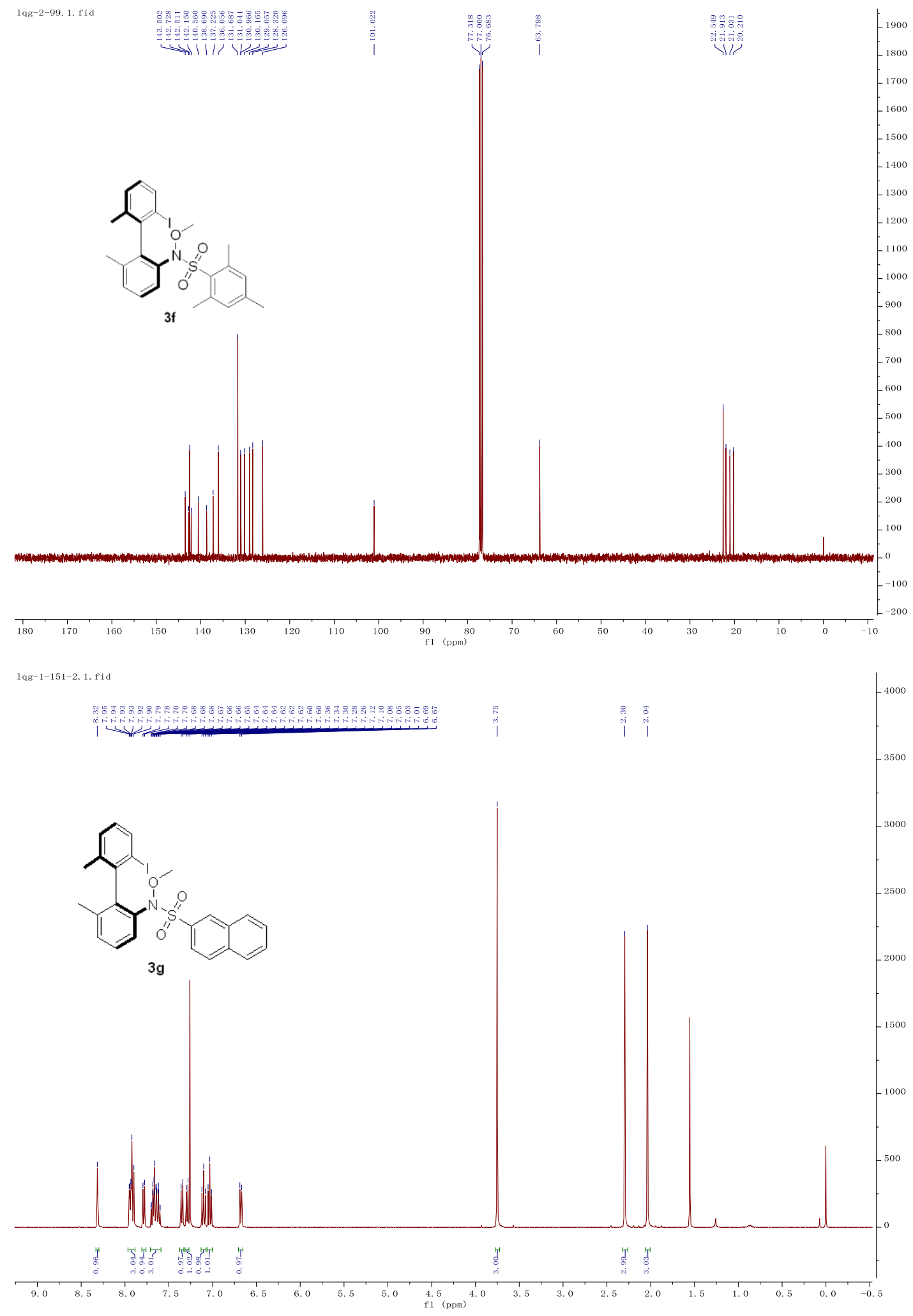




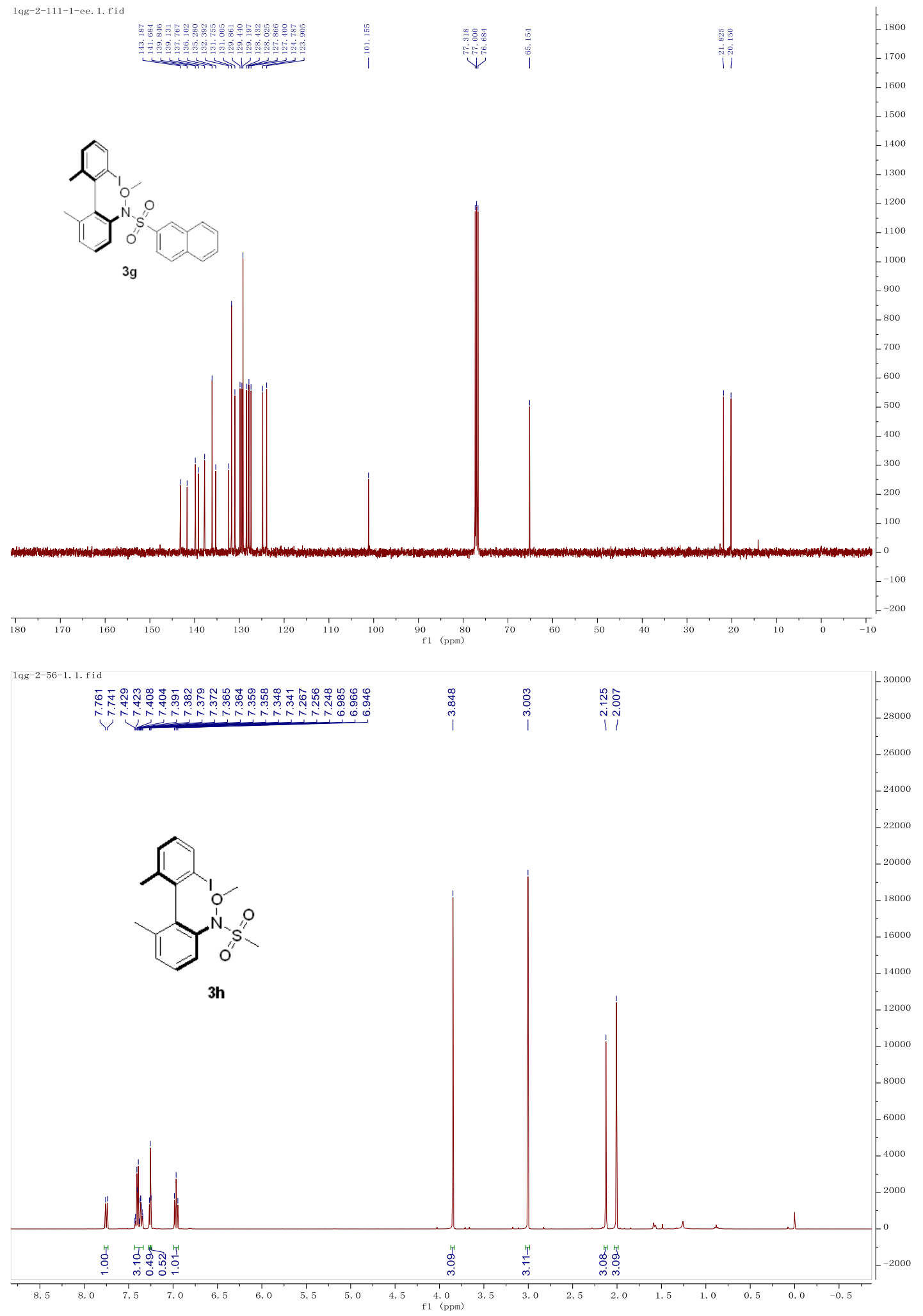




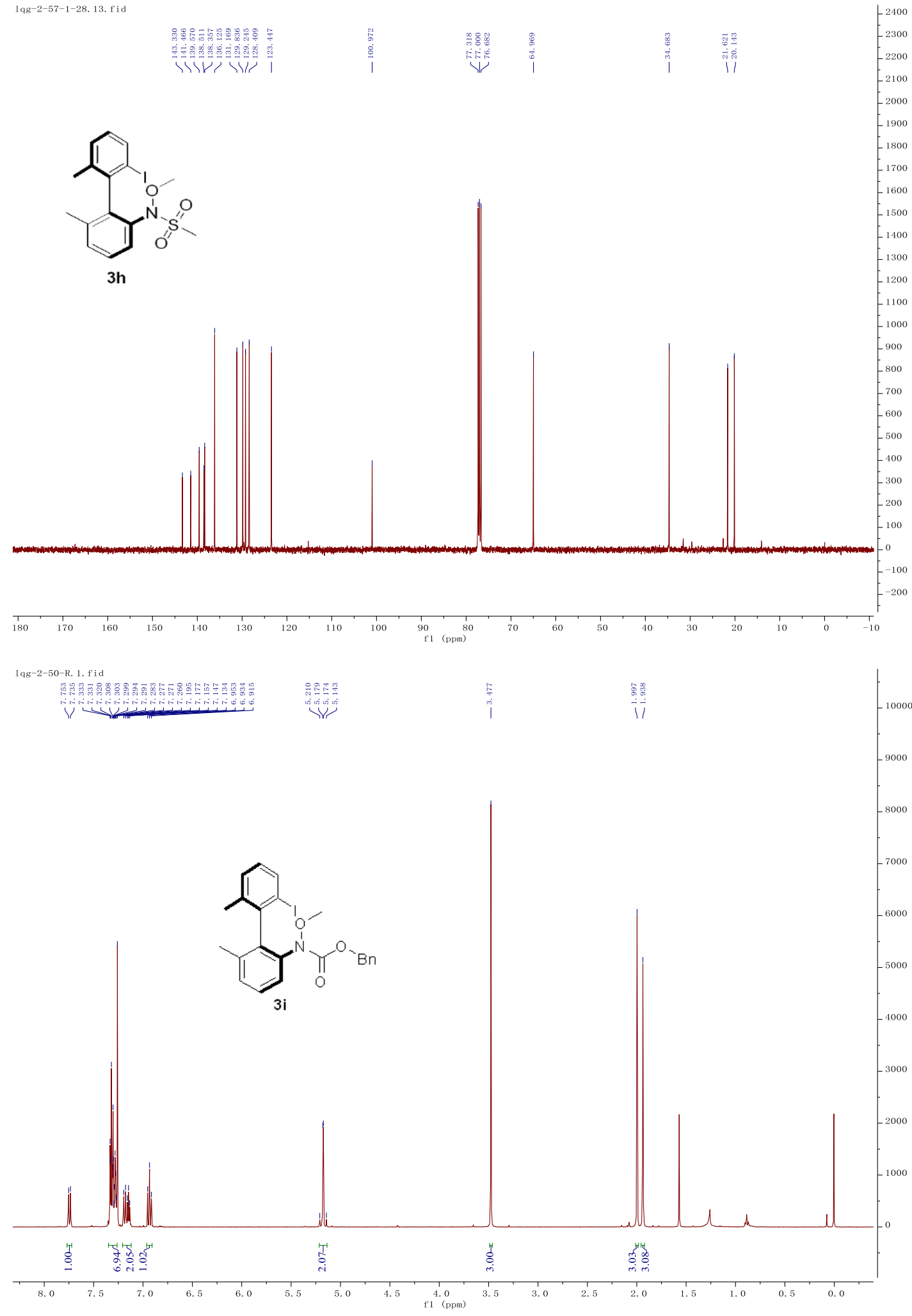




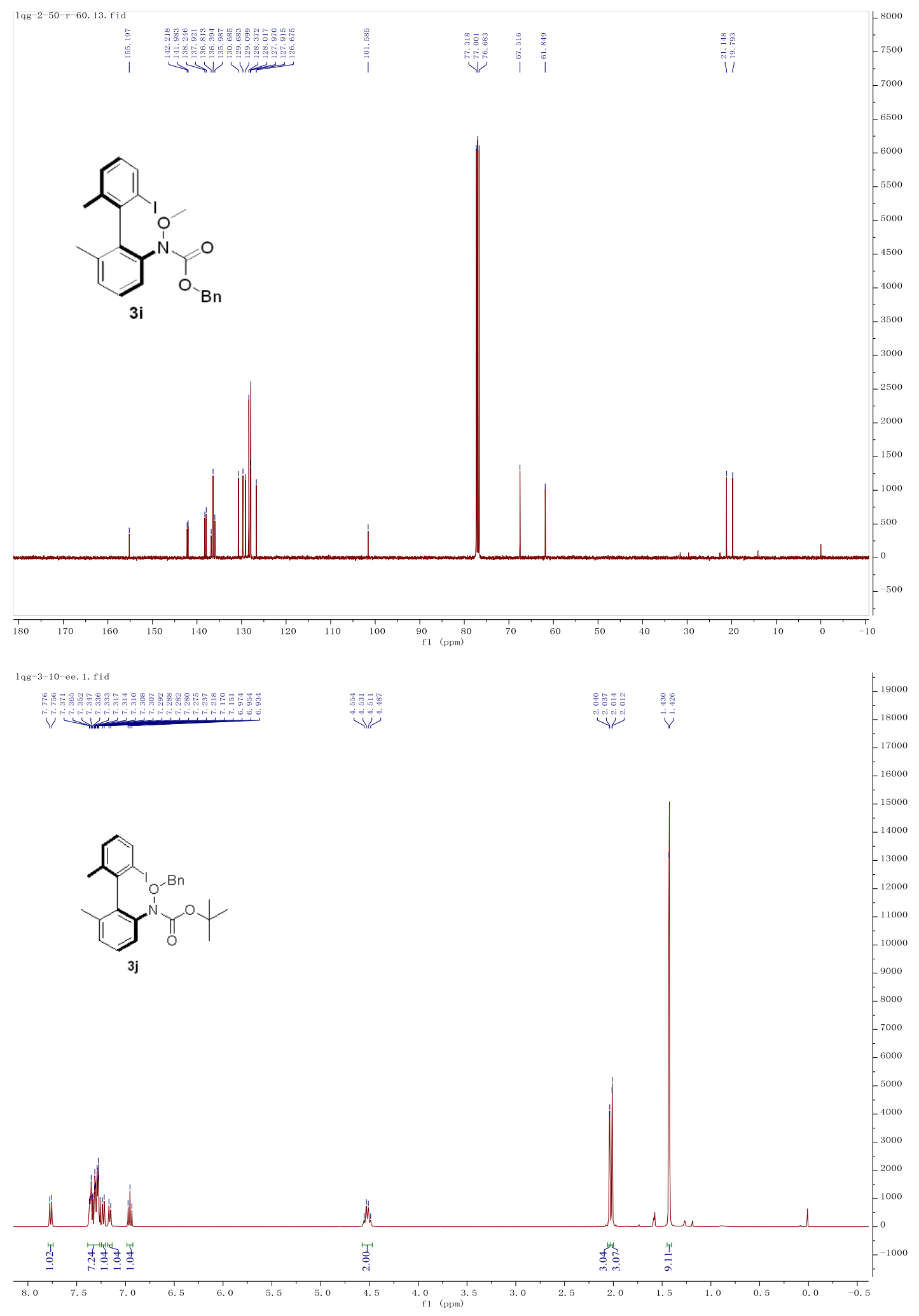




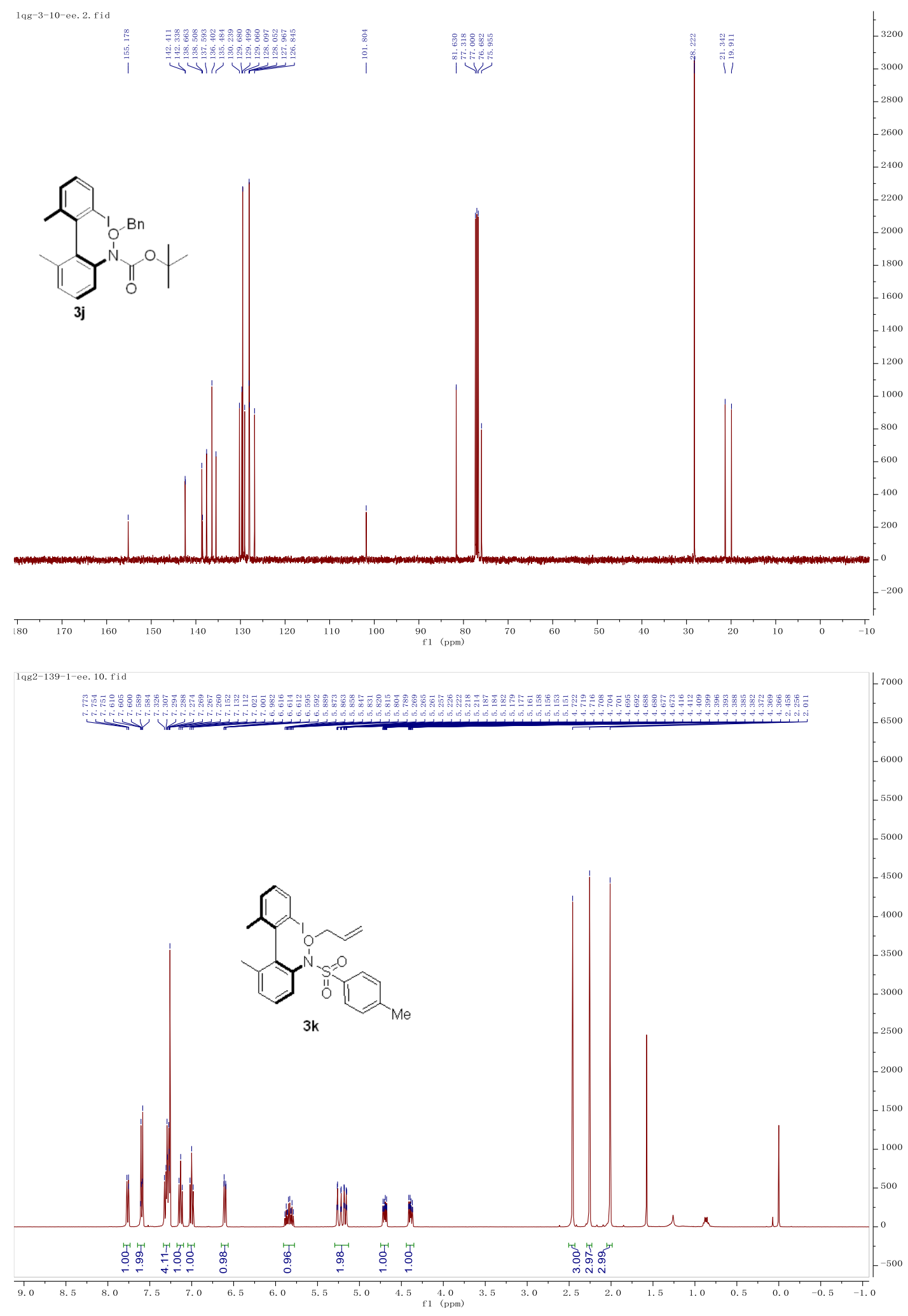




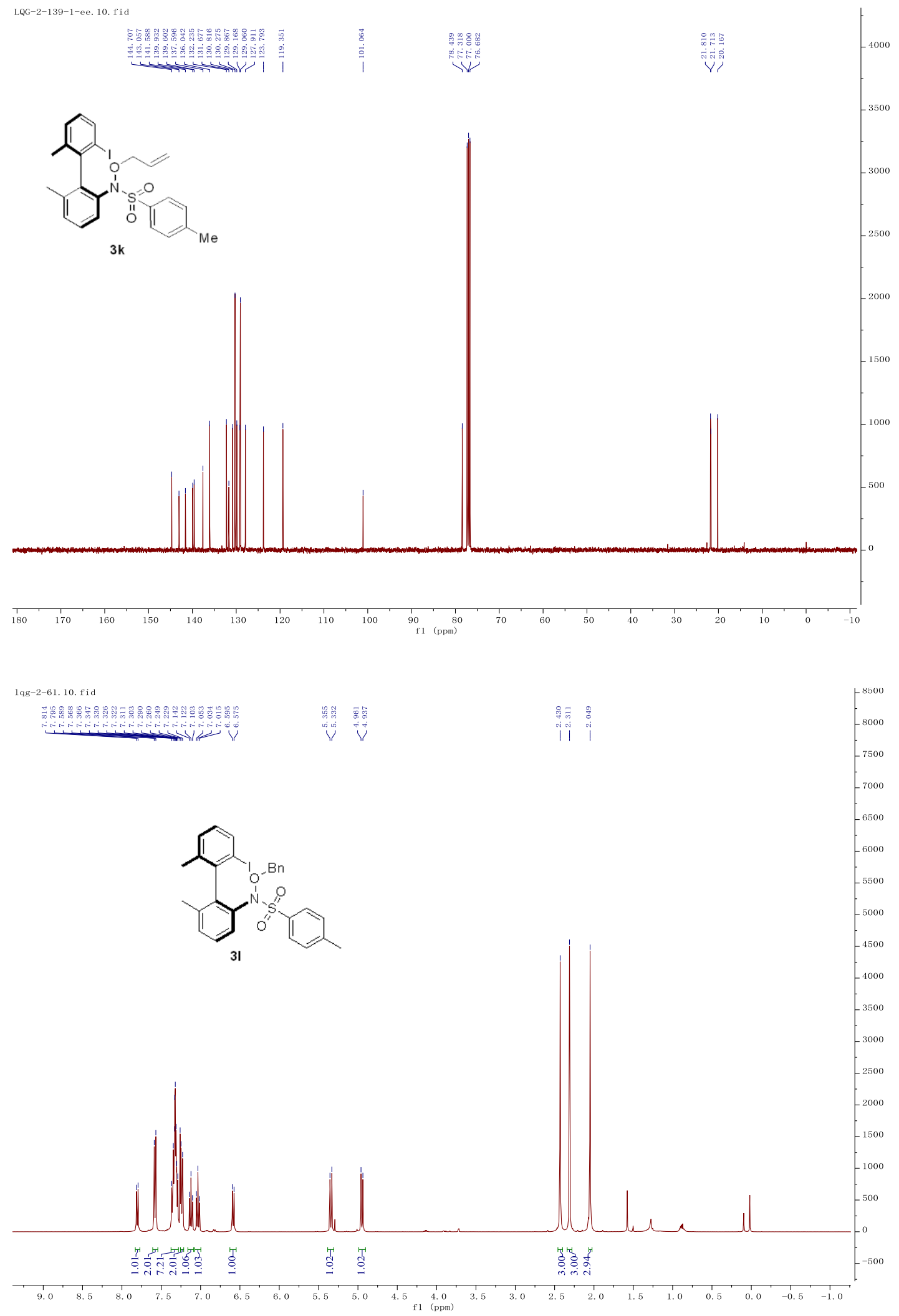



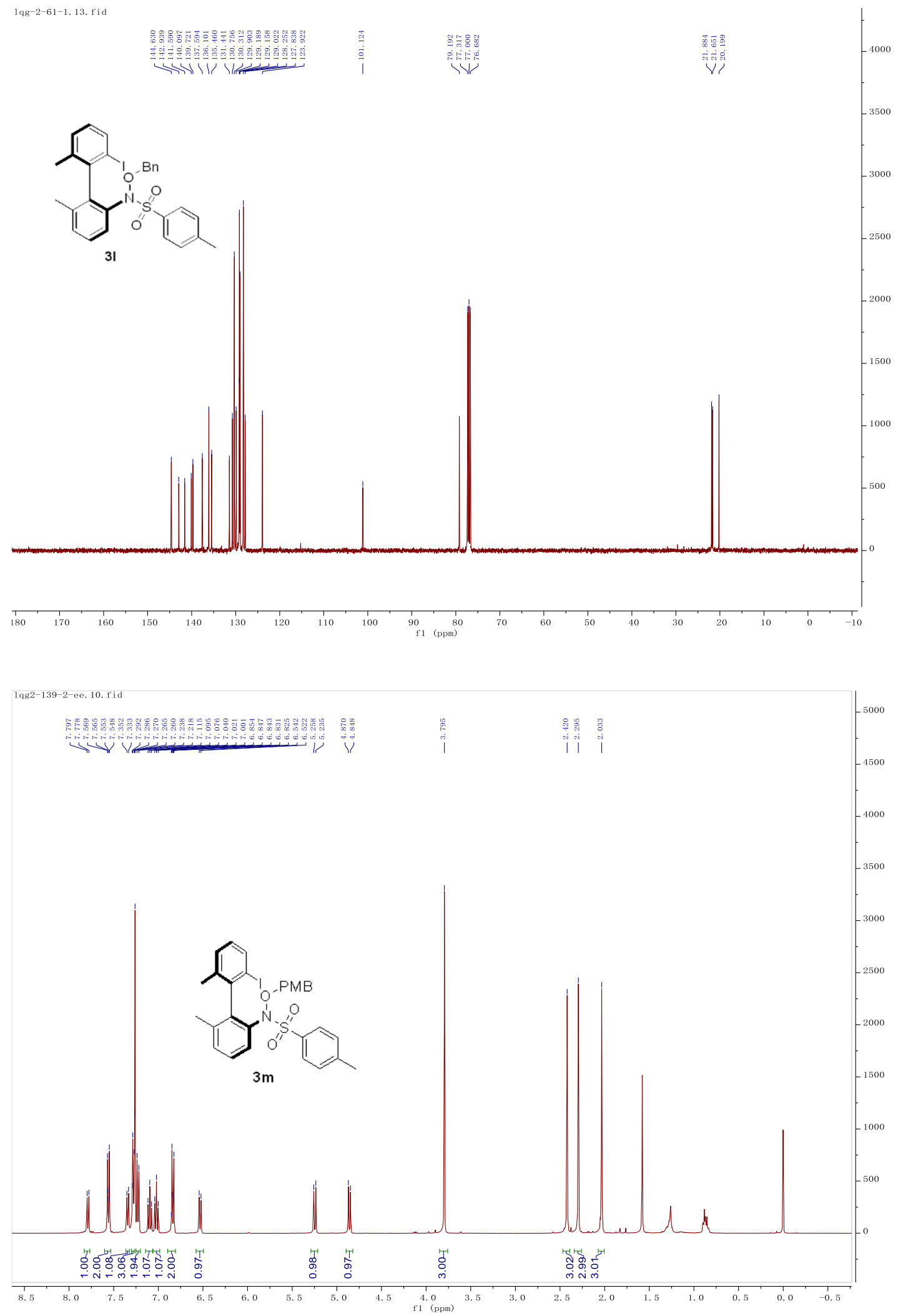


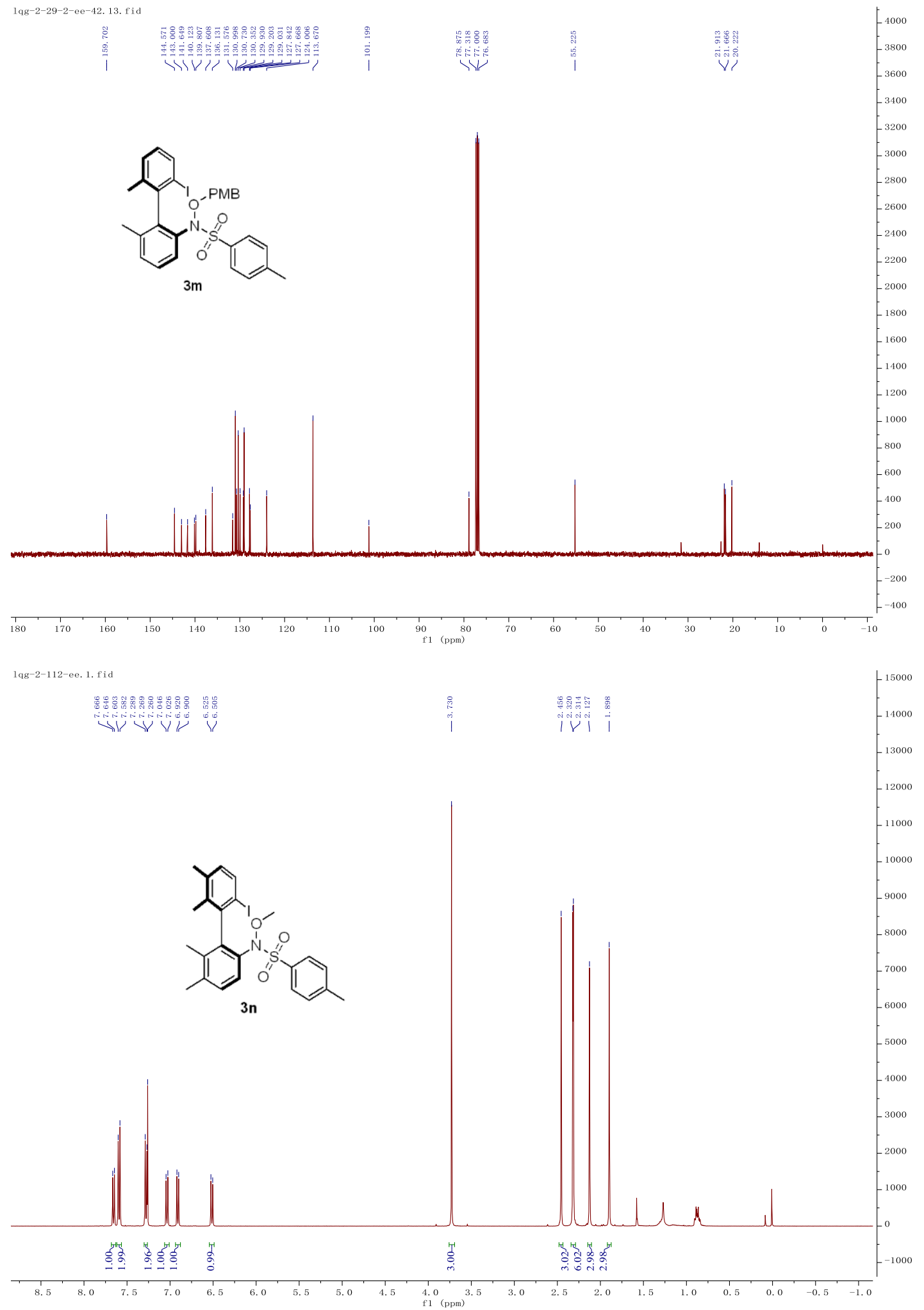




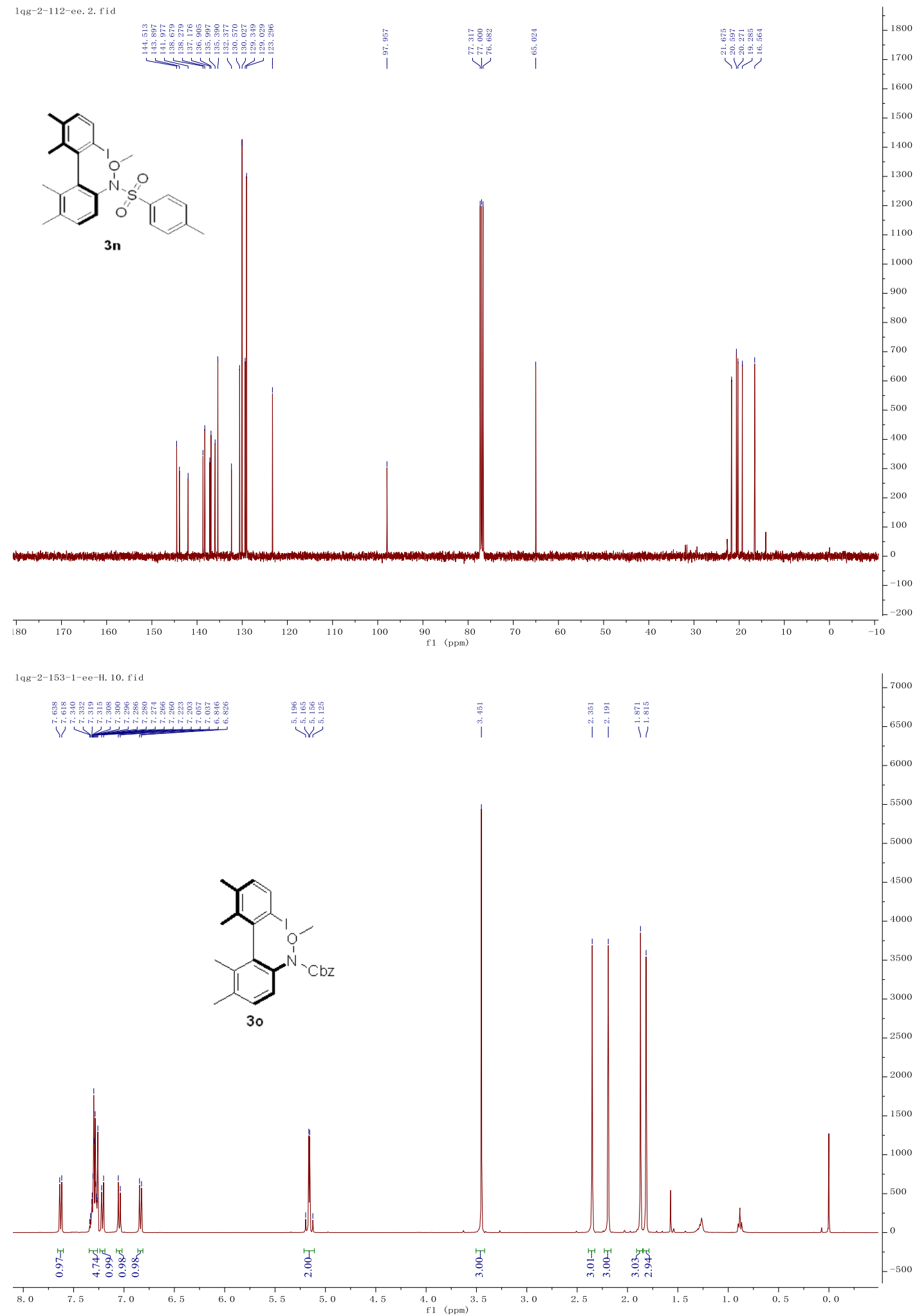




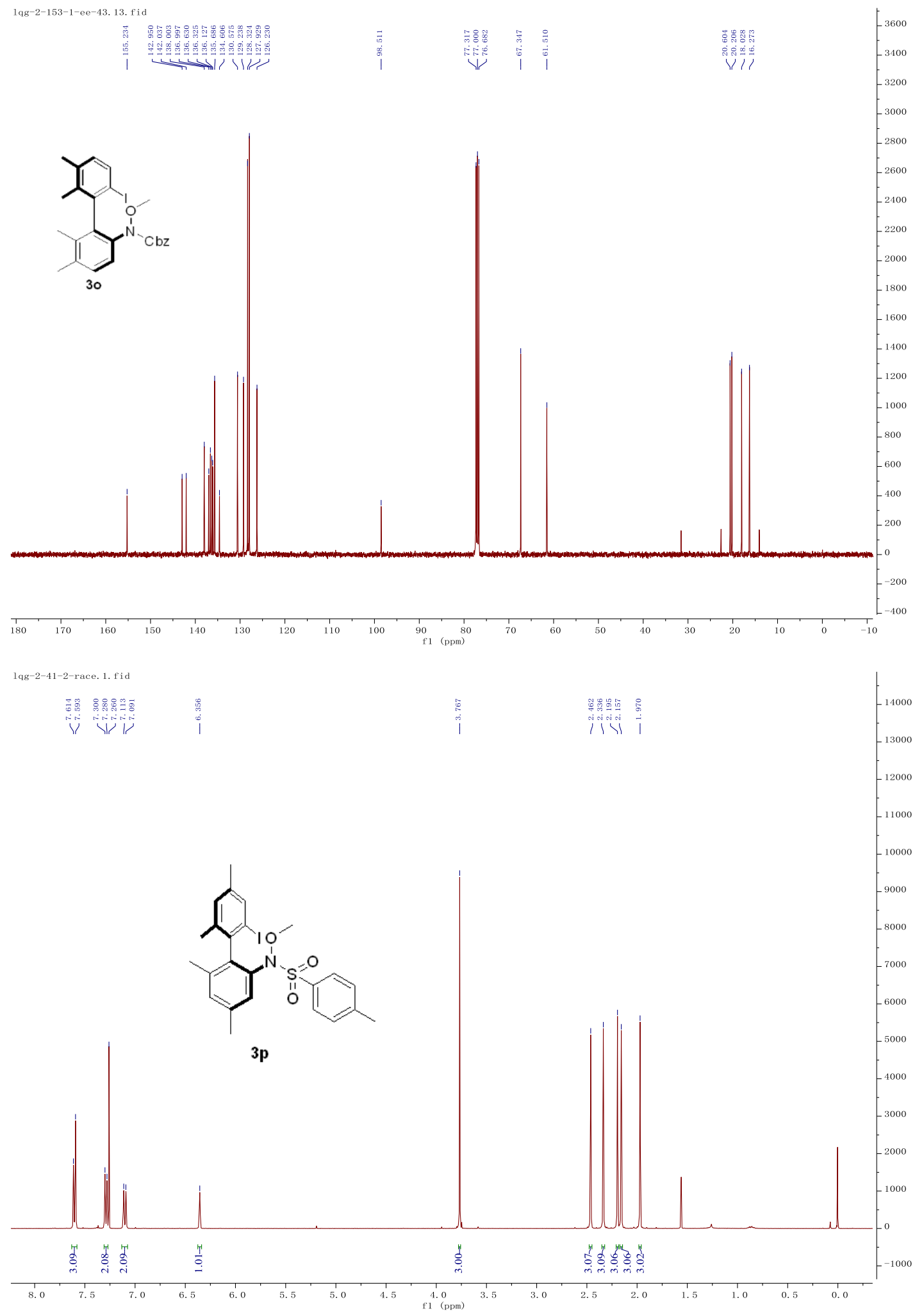




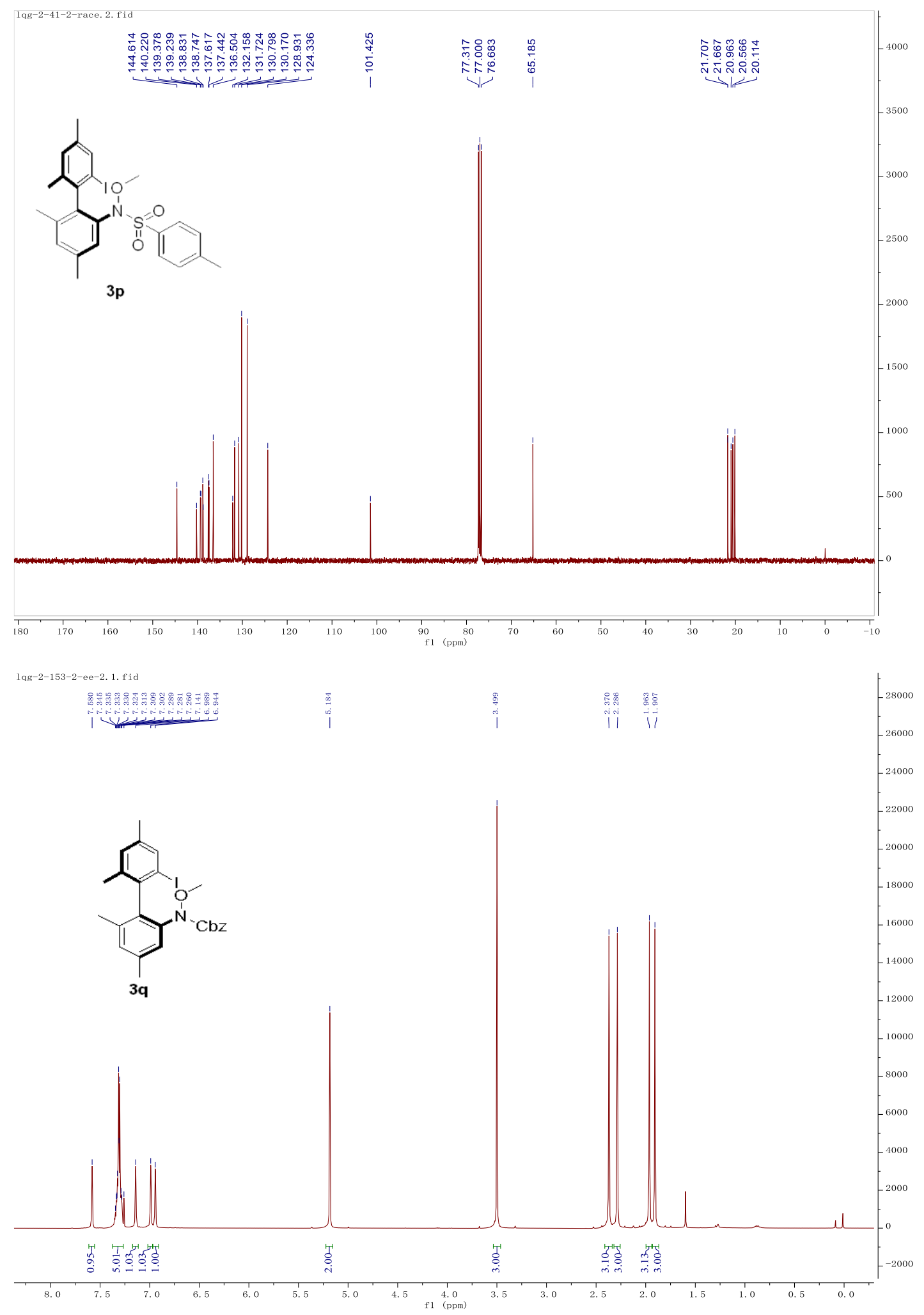




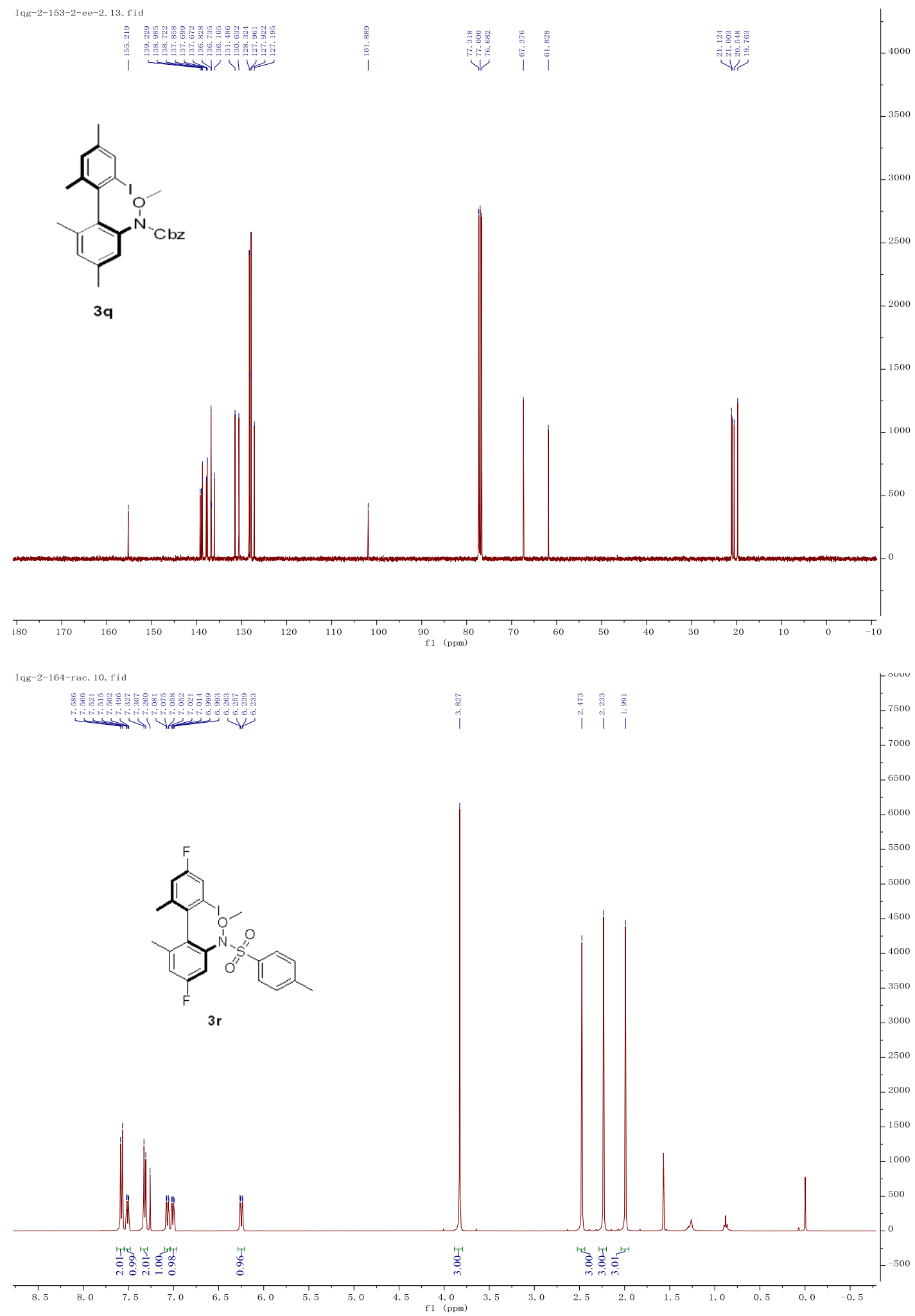



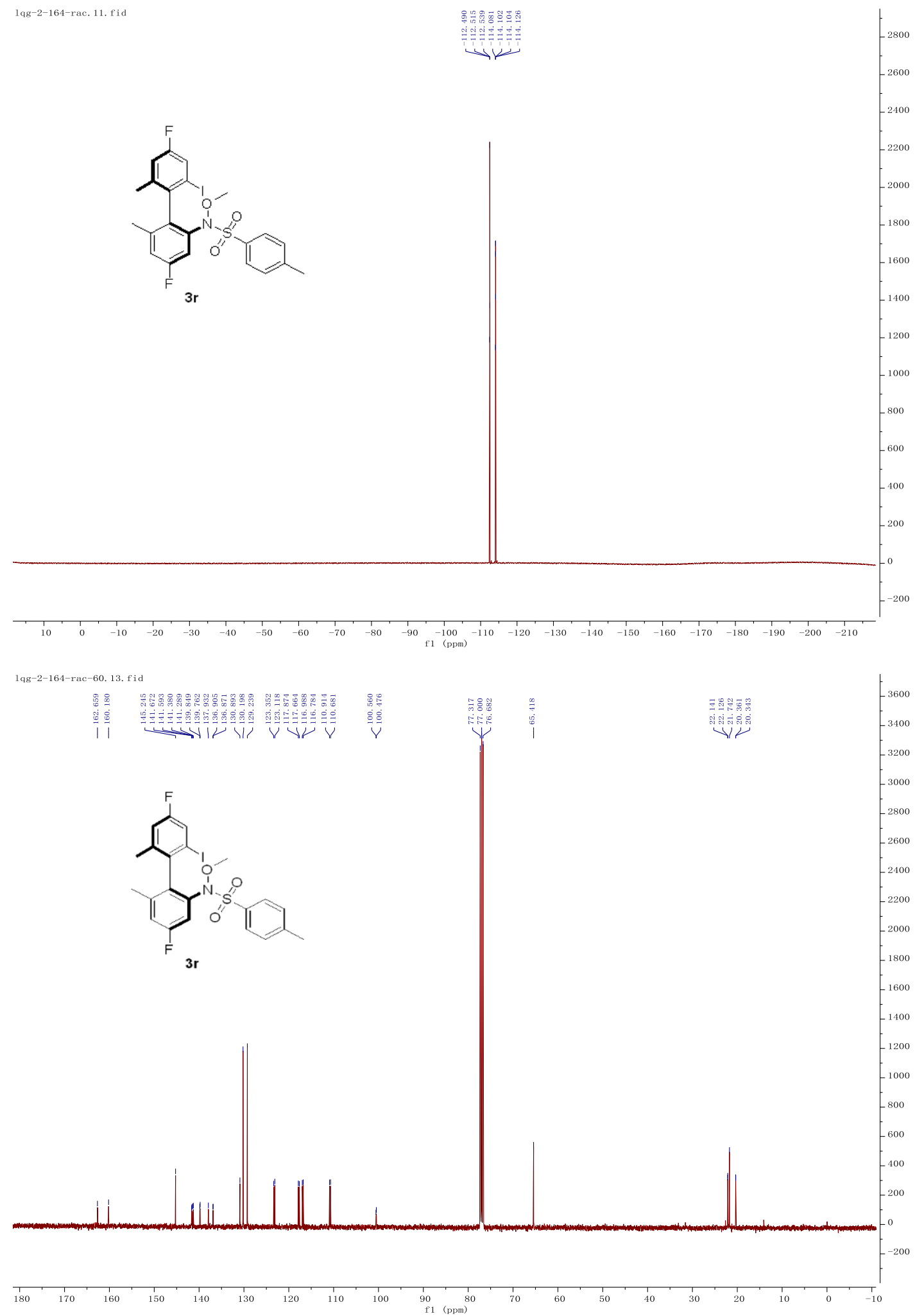

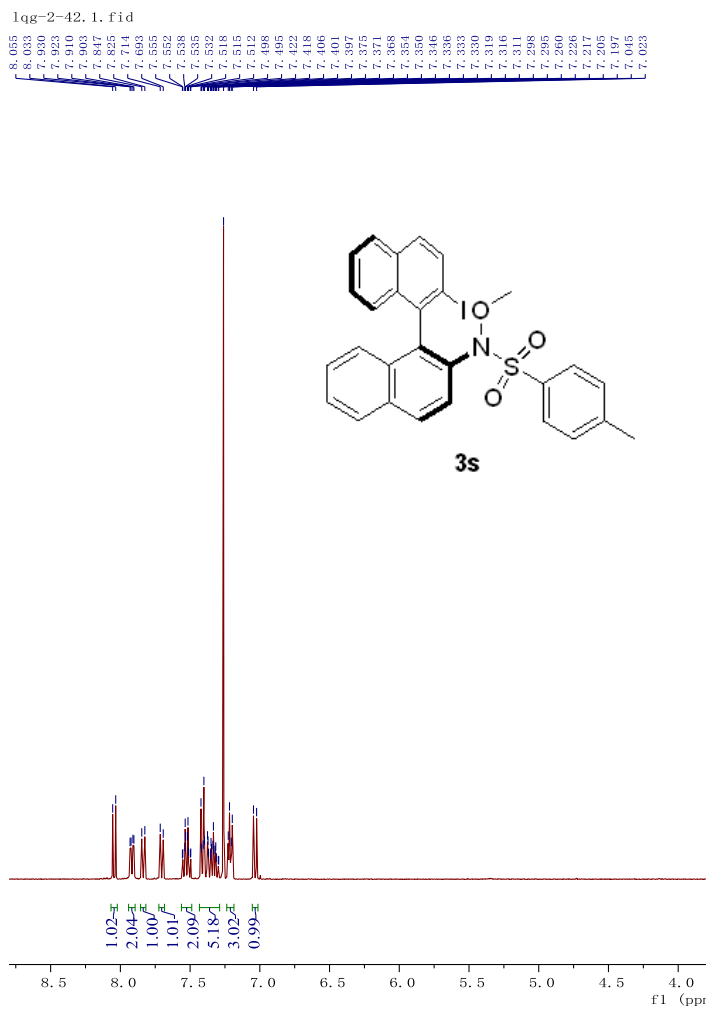

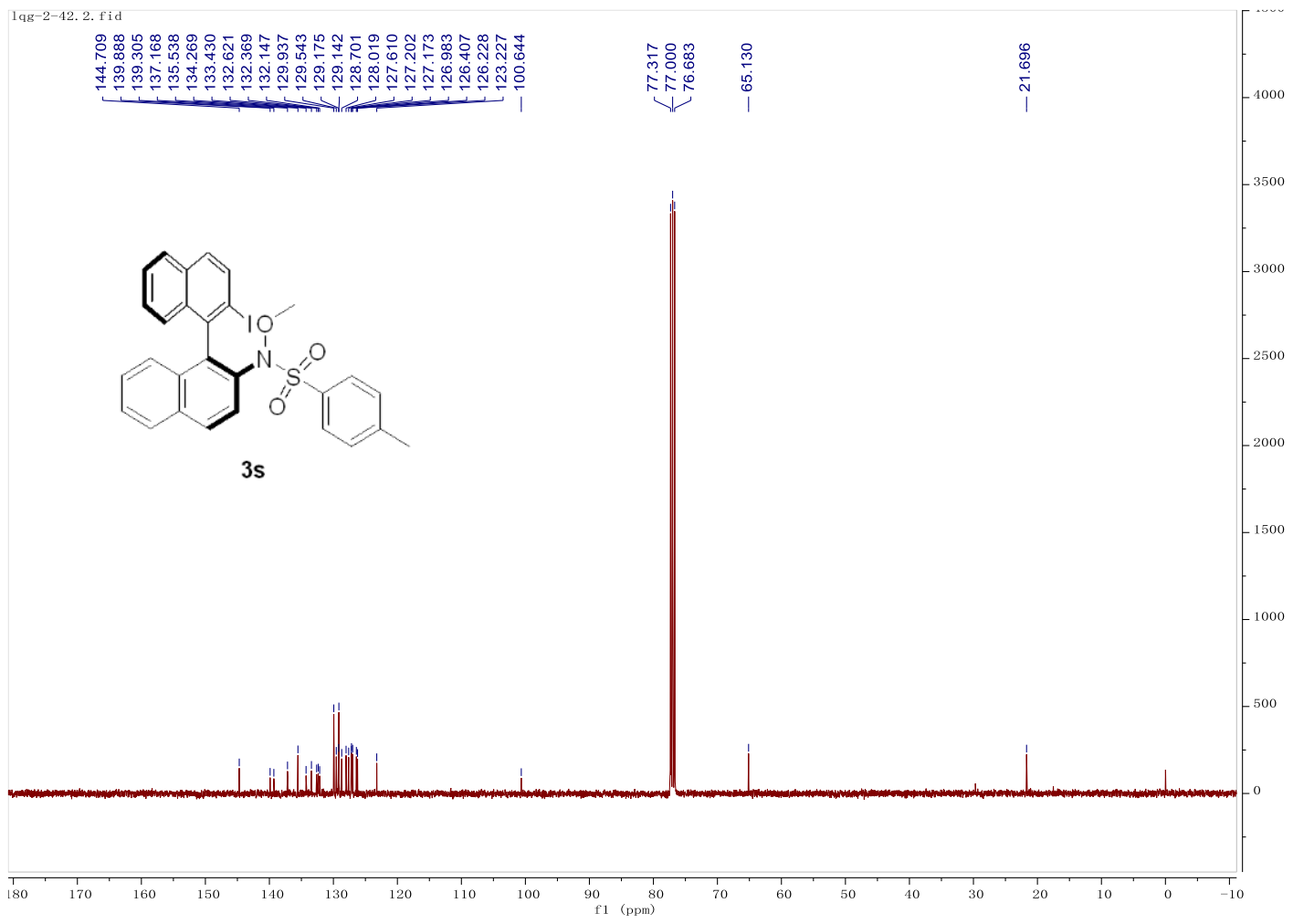

S34 


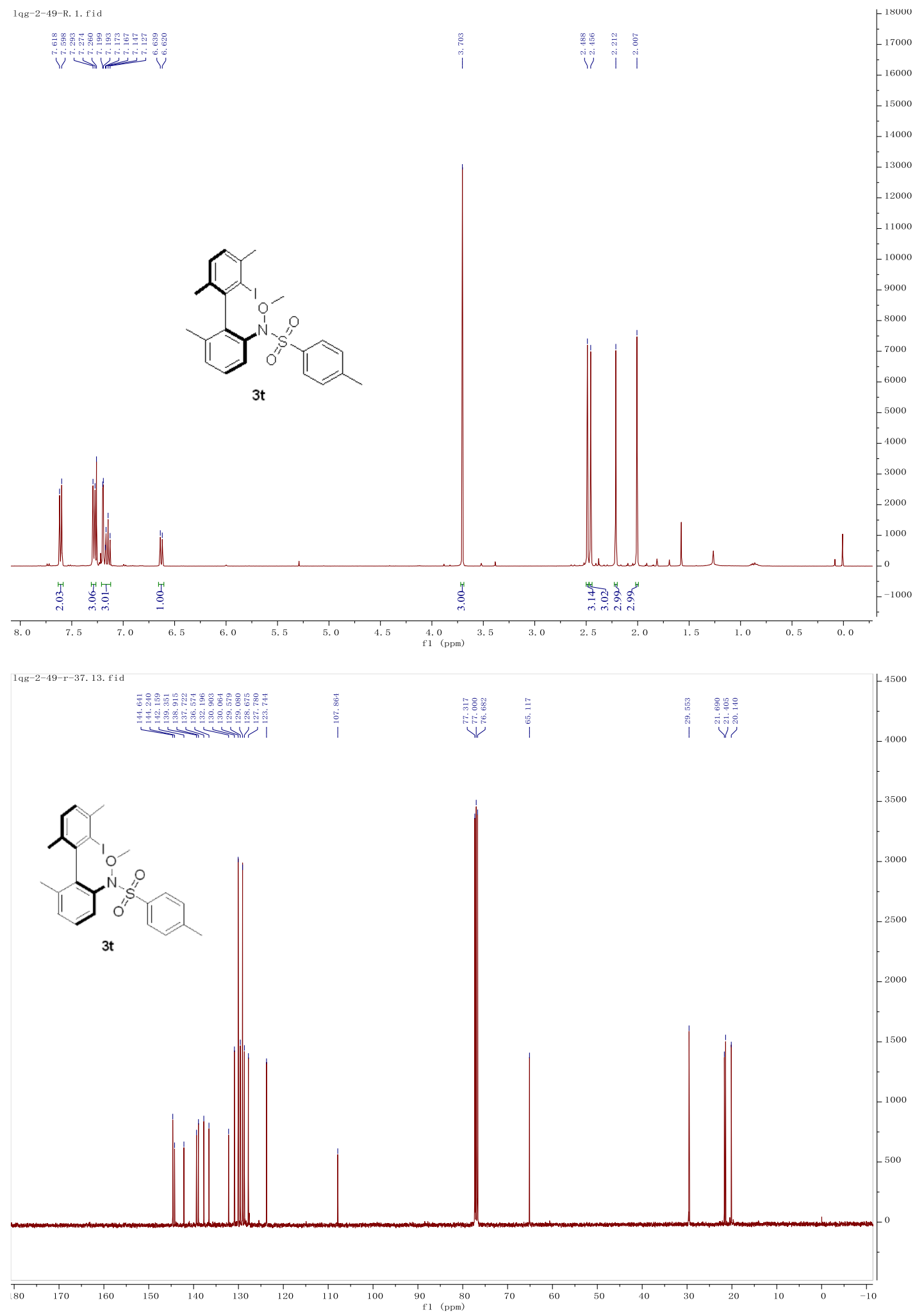




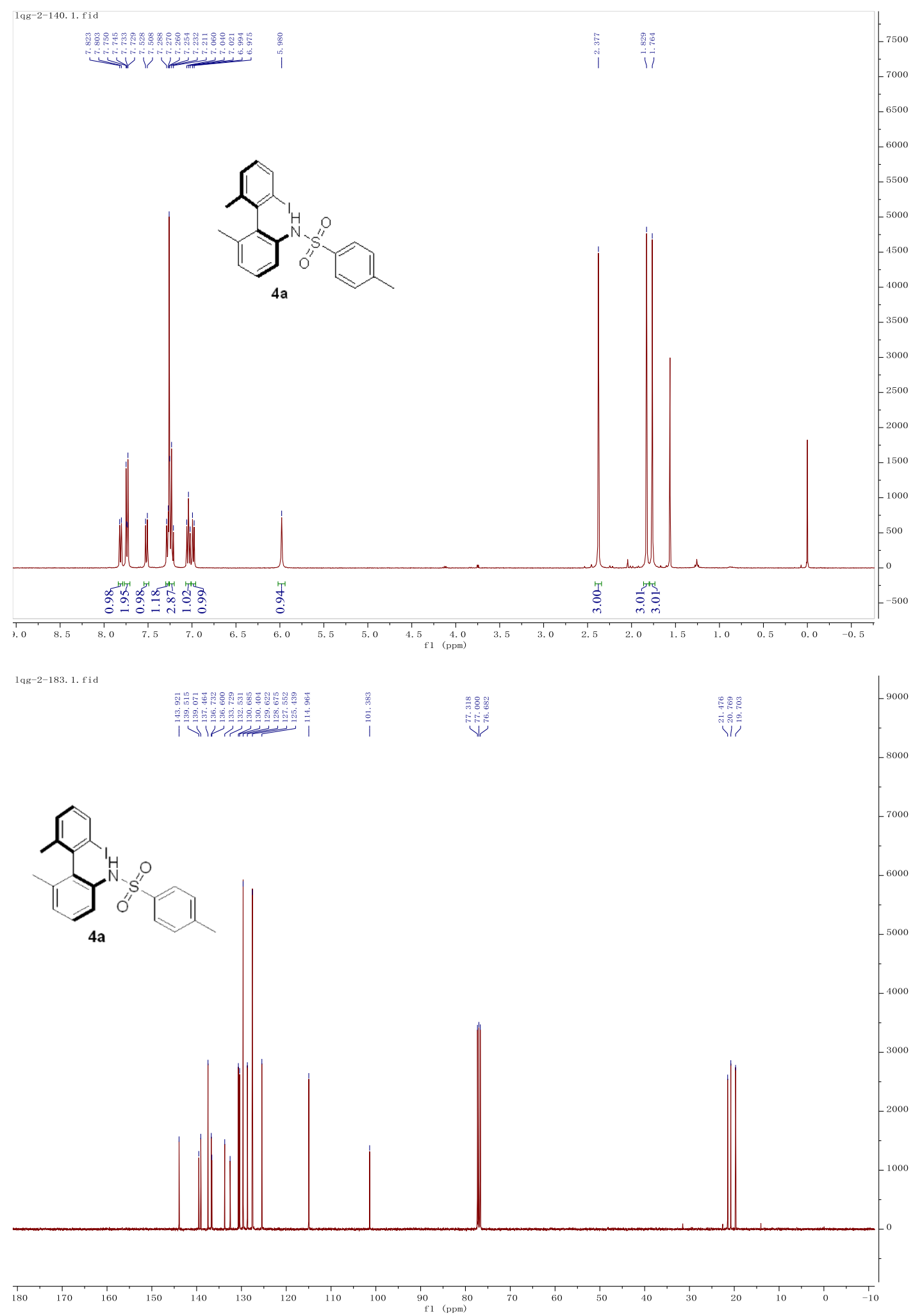




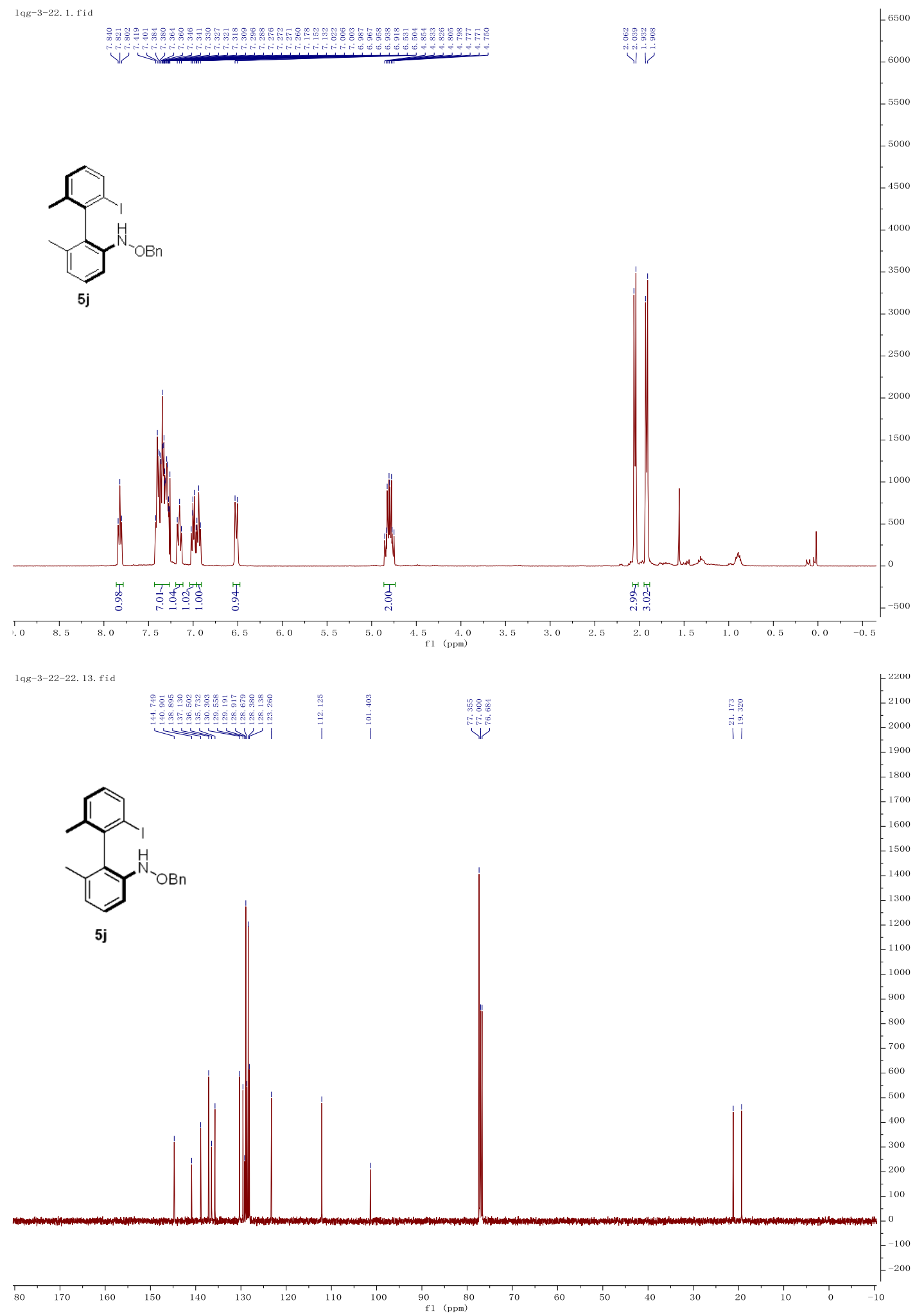




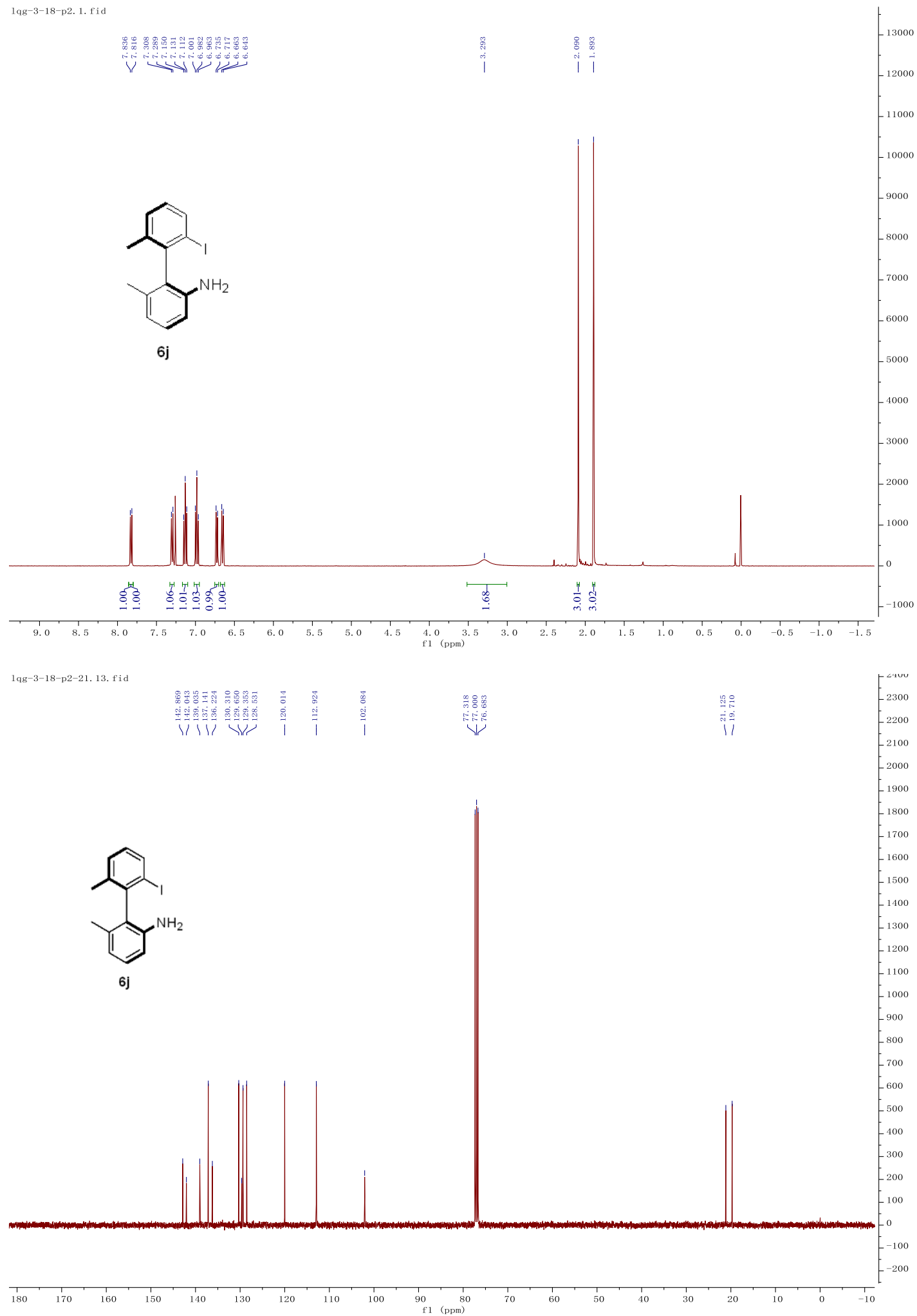



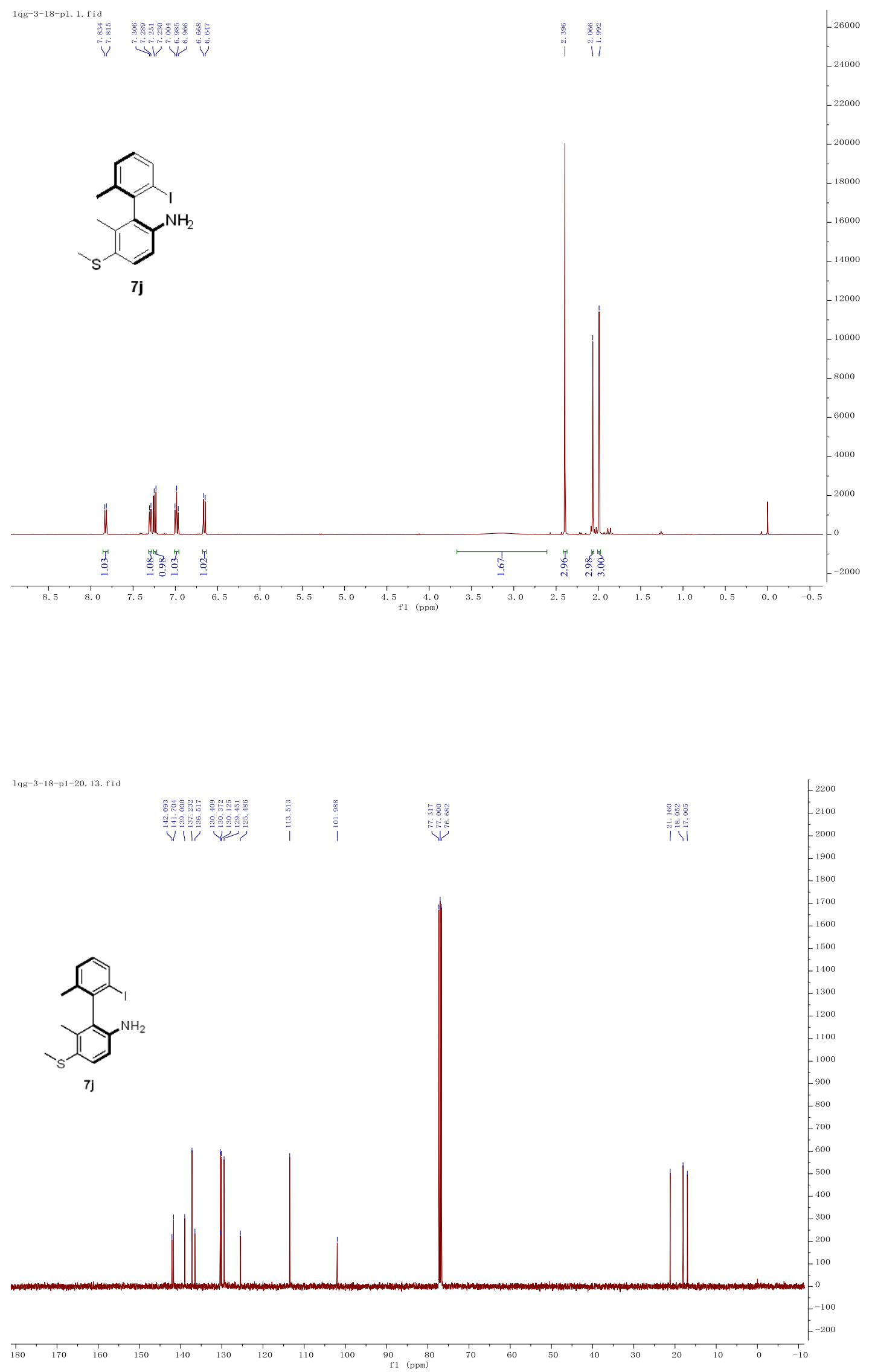


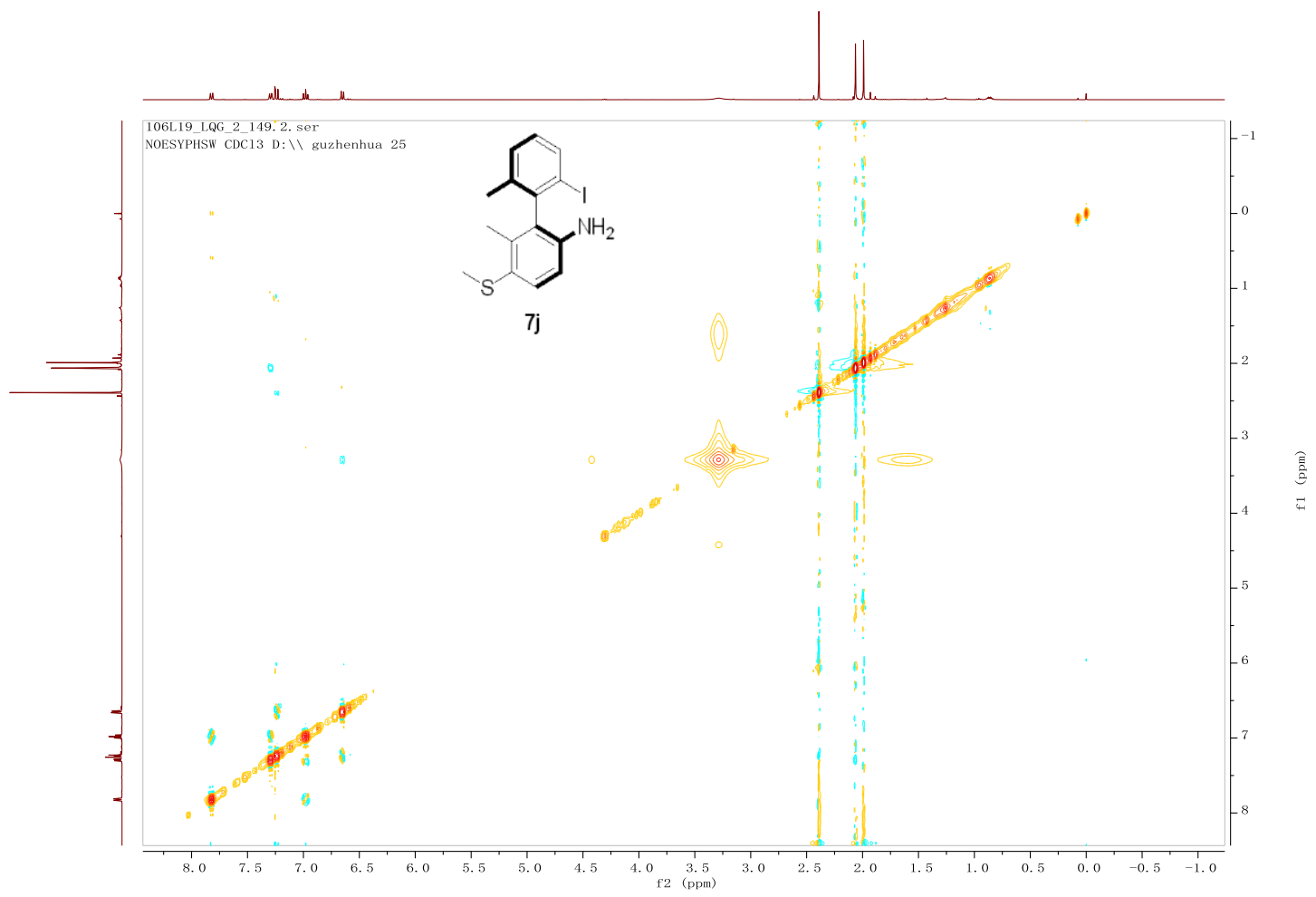

S40 


\section{Copies of HPLC Traces}

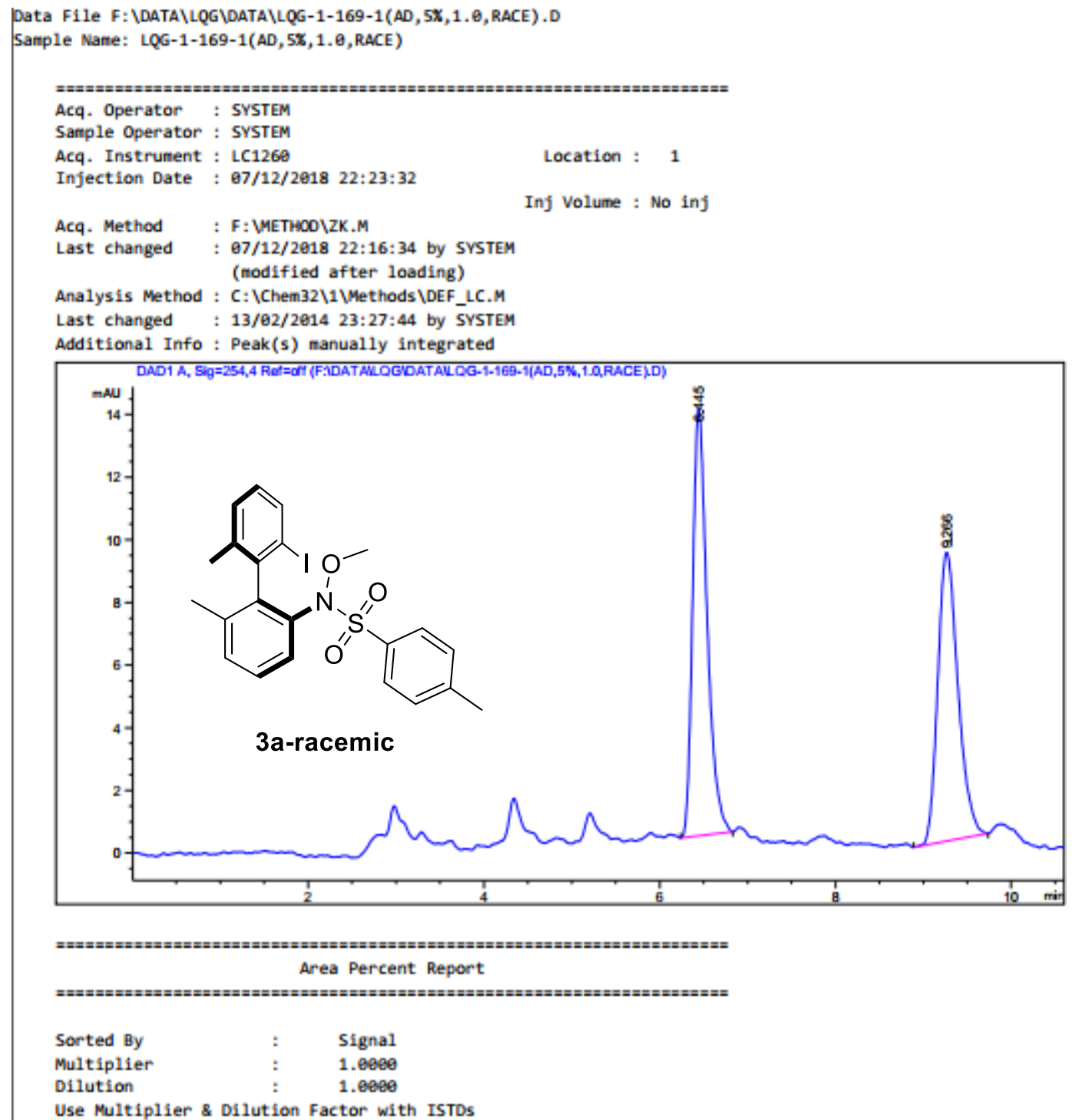

Signal 1: DAD1 A, Sig $=254,4$ Ref $=$ off

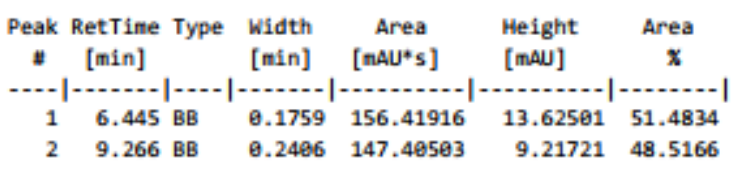

$\begin{array}{lll}\text { Totals : } & 303.82419 & 22.84222\end{array}$ 


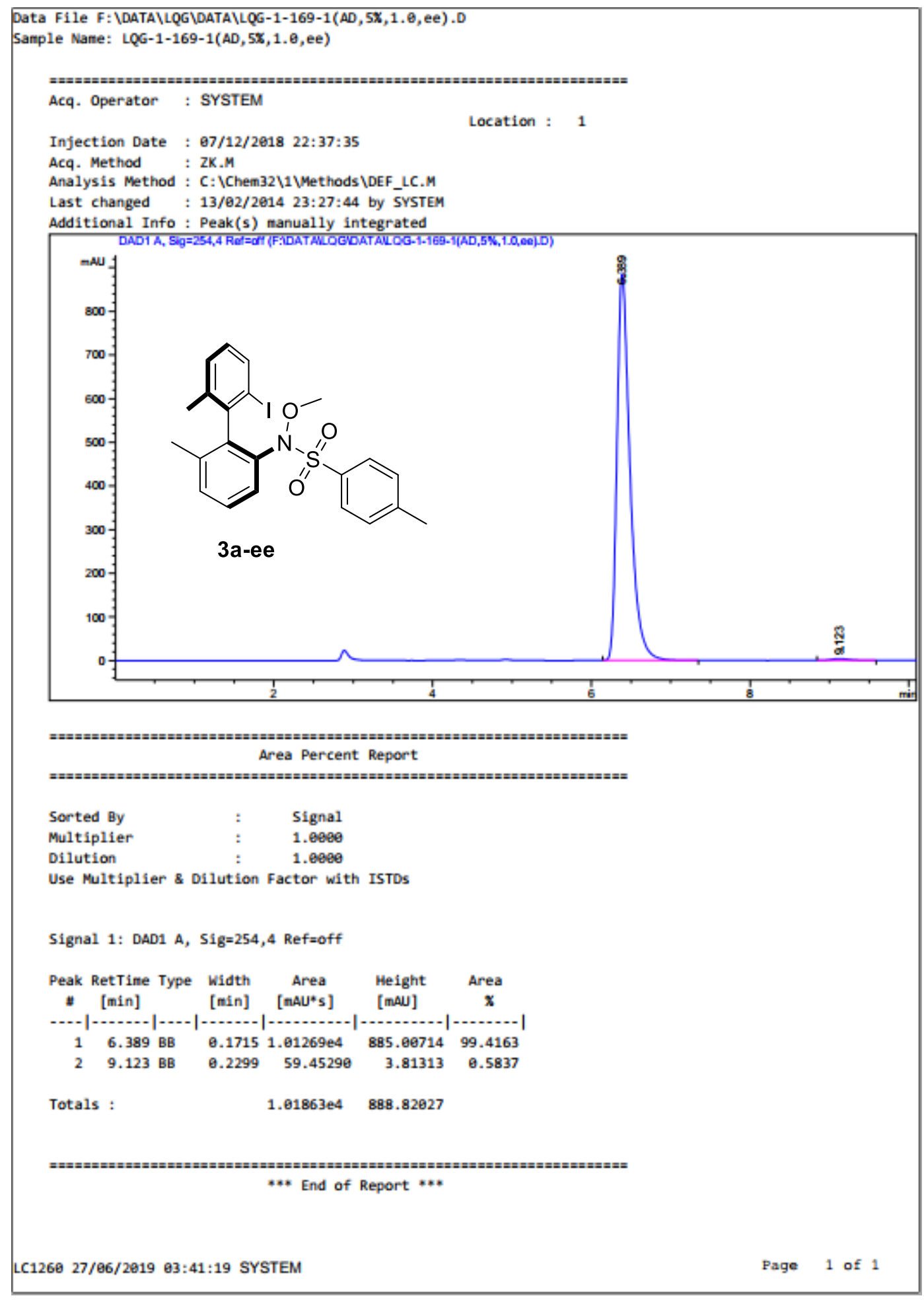




\section{<Sample Information>}

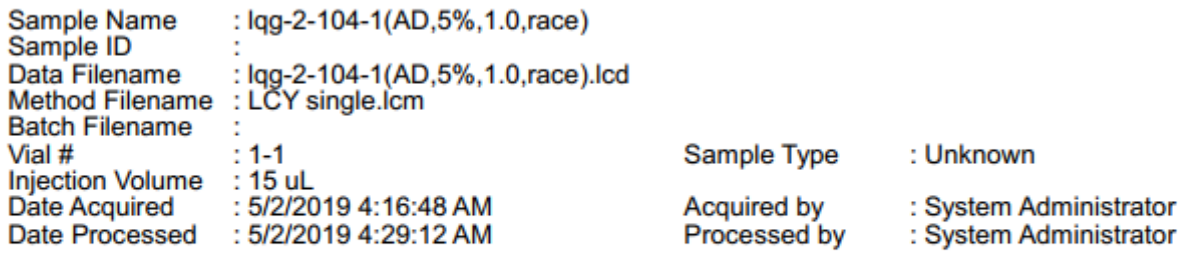

Batch Filename

Vial \#

Date Acquired : :5/2/2019 4:16:48 AM

Date Processed $\quad: 5 / 2 / 2019$ 4:29:12 AM

Sample Type : Unknown

Acquired by : : System Administrator Processed by : System Administrator

\section{$<$ Chromatogram $>$}

$\mathrm{mV}$

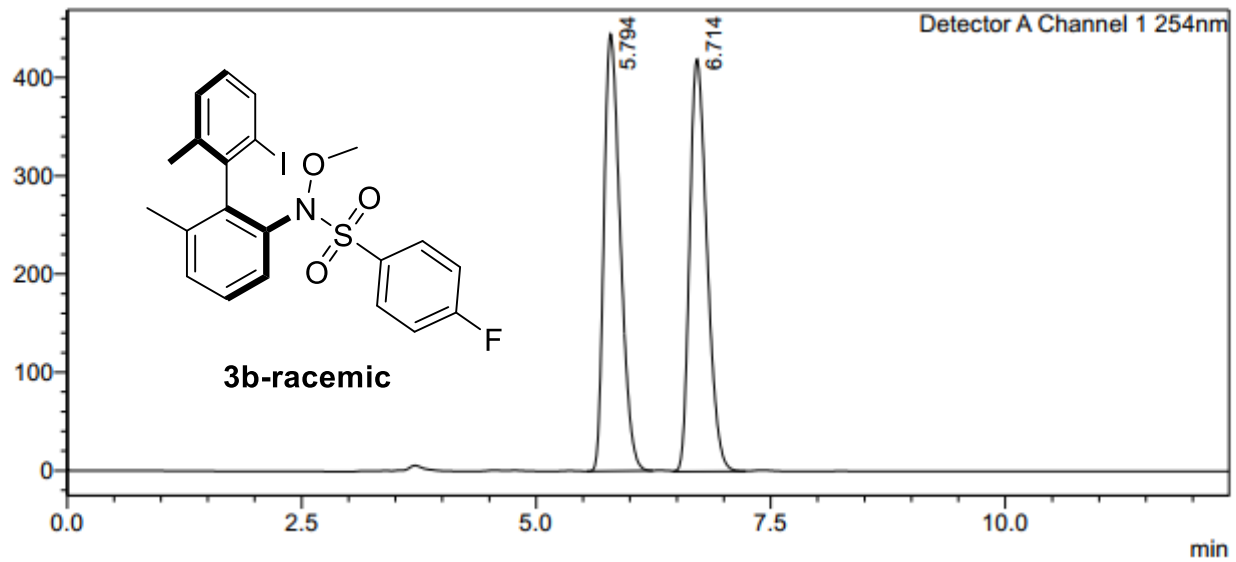

<Peak Table>

Detector A Channel $1254 \mathrm{~nm}$

\begin{tabular}{|r|r|r|r|r|r|r|r|}
\hline Peak\# & Ret. Time & \multicolumn{1}{c|}{ Area } & Height & Conc. & Unit & Mark & Name \\
\hline 1 & 5.794 & 5392930 & 444498 & 49.883 & & $\mathrm{M}$ & \\
\hline 2 & 6.714 & 5418155 & 419538 & 50.117 & & & \\
\hline Total & & 10811085 & 864036 & & & & \\
\hline
\end{tabular}


<Sample Information>

Sample Name $\quad$ : lqg-2-104-1(AD,5\%,1.0,ee)

Sample ID

(

Method Filename : LCY single.lcm

Batch Filename :

Vial \# $: 1-1$

Injection Volume : : 15 uL

Date Acquired :5/2/2019 4:31:45 AM

Sample Type : Unknown

Date Processed $\quad: 5 / 2 / 2019$ 4:39:39 AM

Acquired by : System Administrator

Processed by : System Administrator

\section{$<$ Chromatogram>}

$\mathrm{mV}$

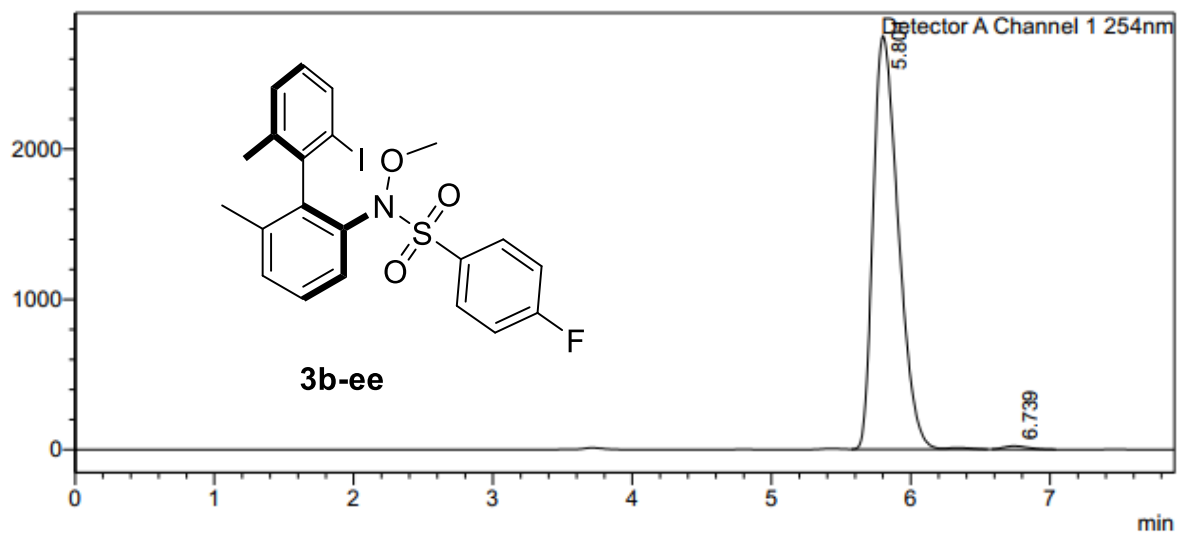

<Peak Table>

Detector A Channel $1254 \mathrm{~nm}$

\begin{tabular}{|r|r|r|r|r|r|r|r|}
\hline Peak\# & Ret. Time & \multicolumn{1}{c|}{ Area } & \multicolumn{1}{c|}{ Height } & \multicolumn{1}{c|}{ Conc. } & Unit & Mark & Name \\
\hline 1 & 5.801 & 34578484 & 2749713 & 99.252 & & $\mathrm{M}$ & \\
\hline 2 & 6.739 & 260493 & 21855 & 0.748 & & $\mathrm{M}$ & \\
\hline Total & & 34838977 & 2771569 & & & & \\
\end{tabular}


<Sample Information>
Sample Name
: lqg-2-110(AD,5\%,1.0,race)
Sample ID
Data Filename
: Igg-2-110(AD,5\%,1.0, race).Icd
Method Filename : GWJ single.Icm
Batch Filename
Vial \#
$: 1-1$
: : : 5/3/2019 12:34:05 AM
Sample Type : Unknown
Date Processed $\quad: 5 / 3 / 2019$ 12:53:14 AM
Acquired by : : System Administrator
Processed by : System Administrator

\section{$<$ Chromatogram>}

$\mathrm{mV}$

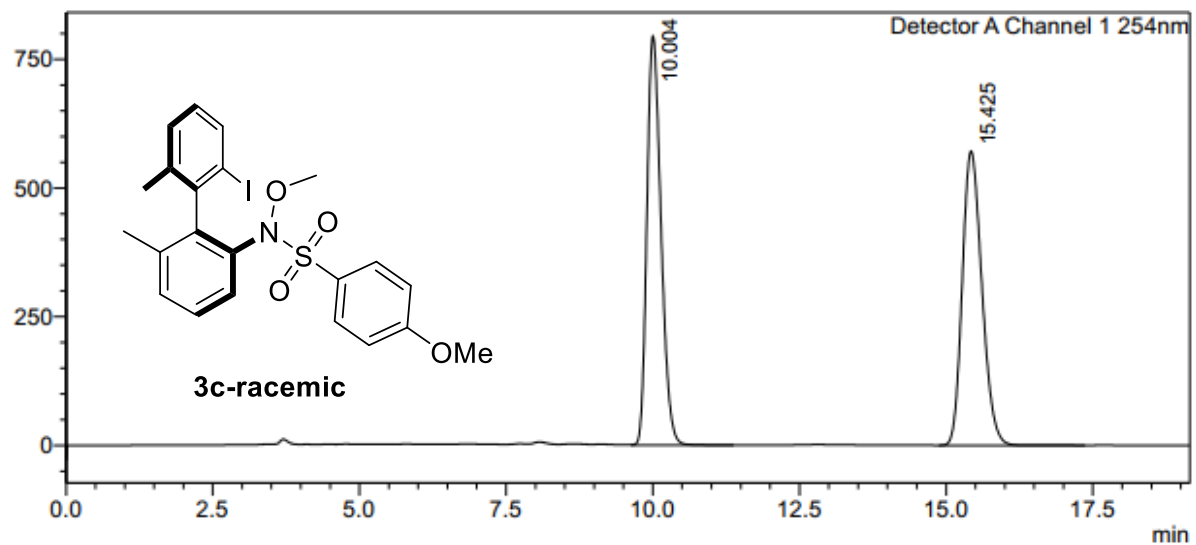

<Peak Table>

Detector A Channel $1254 \mathrm{~nm}$

\begin{tabular}{|r|r|r|r|r|r|r|r|}
\hline Peak\# & Ret. Time & \multicolumn{1}{c|}{ Area } & \multicolumn{1}{c|}{ Height } & \multicolumn{1}{c|}{ Conc. } & Unit & Mark & Name \\
\hline 1 & 10.004 & 13295279 & 794421 & 49.983 & & $\mathrm{~S}$ & \\
\hline 2 & 15.425 & 13304399 & 571315 & 50.017 & & & \\
\hline Total & & 26599678 & 1365735 & & & & \\
\hline
\end{tabular}




\section{<Sample Information>}

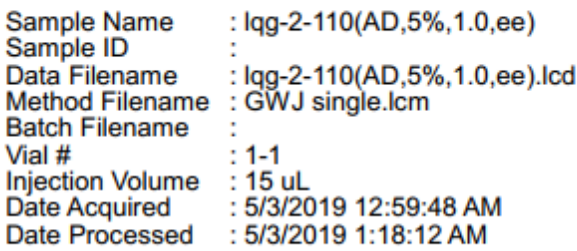

Date Acquired $\quad: 5 / 3 / 2019$ 12:59:48 AM

Date Processed $\quad: 5 / 3 / 2019$ 1:18:12 AM

Sample Type : Unknown

Acquired by : : System Administrato Processed by : : System Administrator

\section{$<$ Chromatogram $>$}

$\mathrm{mV}$

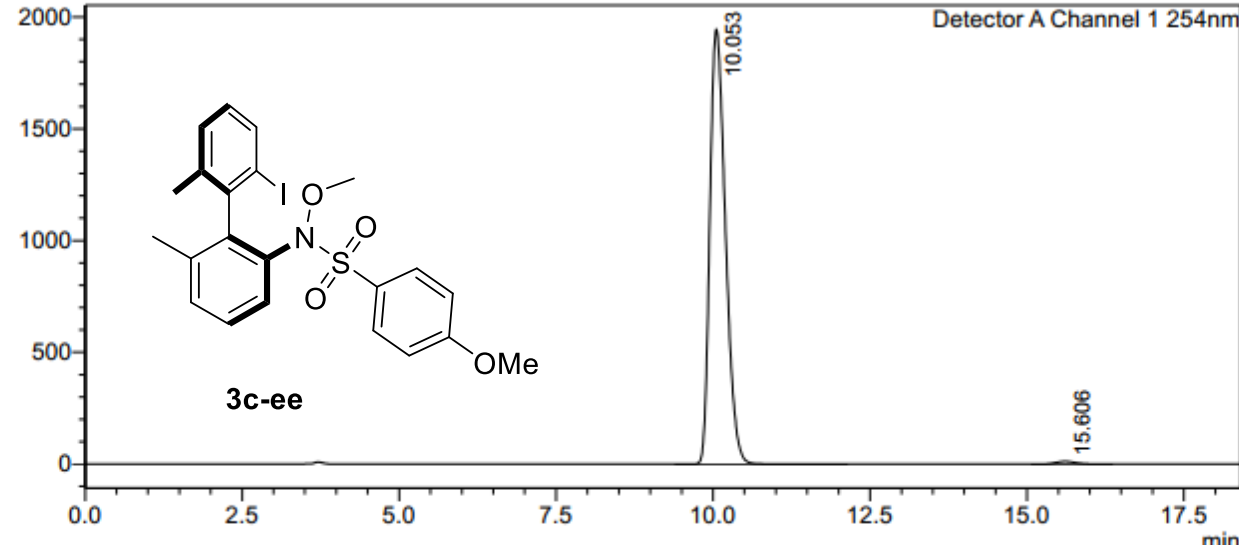

<Peak Table>

Detector A Channel $1254 \mathrm{~nm}$

\begin{tabular}{|r|r|r|r|r|r|r|r|}
\hline Peak\# & Ret. Time & \multicolumn{1}{c|}{ Area } & \multicolumn{1}{c|}{ Height } & \multicolumn{1}{c|}{ Conc. } & Unit & Mark & Name \\
\hline 1 & 10.053 & 33550443 & 1944005 & 99.227 & & S M & \\
\hline 2 & 15.606 & 261243 & 11496 & 0.773 & & & \\
\hline Total & & 33811685 & 1955501 & & & & \\
\hline
\end{tabular}


<Sample Information>

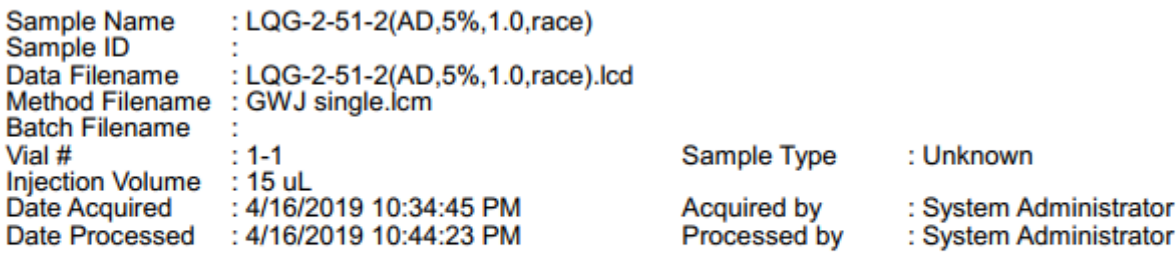

\section{<Chromatogram>}

$\mathrm{mV}$

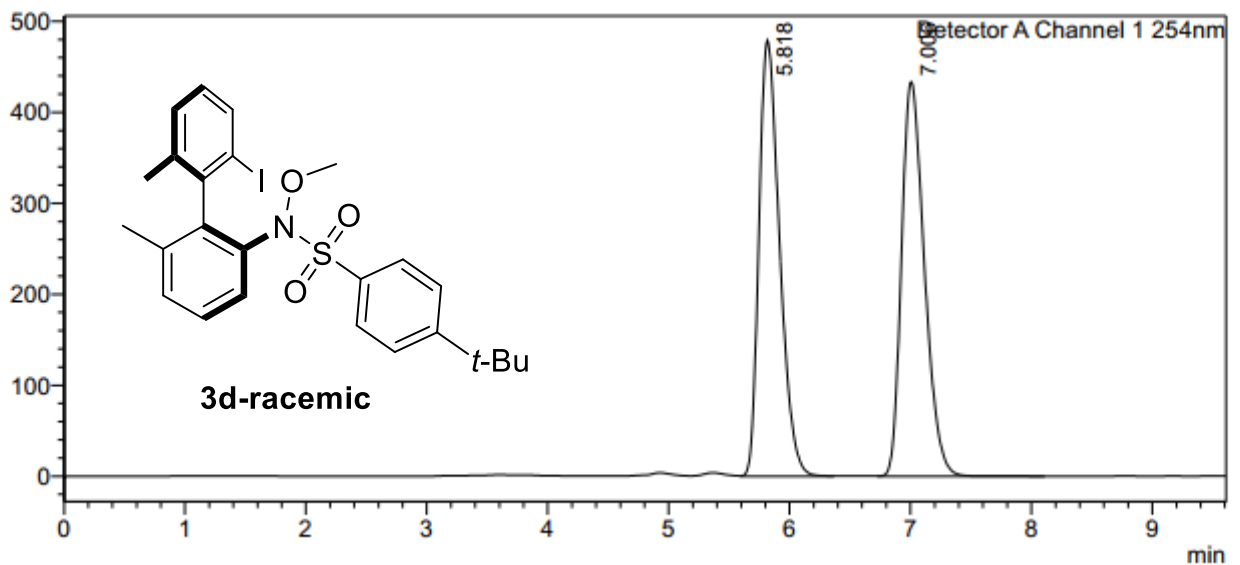

\section{<Peak Table>}

Detector A Channel $1254 \mathrm{~nm}$

\begin{tabular}{|r|r|r|r|r|r|r|r|}
\hline Peak\# & Ret. Time & \multicolumn{1}{|c|}{ Area } & \multicolumn{1}{c|}{ Height } & \multicolumn{1}{c|}{ Conc. } & Unit & Mark & Name \\
\hline 1 & 5.818 & 5684225 & 479095 & 49.940 & & & \\
\hline 2 & 7.006 & 5697828 & 433539 & 50.060 & & & \\
\hline Total & & 11382053 & 912634 & & & & \\
\hline
\end{tabular}


<Sample Information>

$\begin{array}{ll}\text { Sample Name } & \text { : LQG-2-51-2 (2)(AD,5\%,1.0,ee) } \\ \text { Sample ID } & : \\ \text { Data Filename } & \text { : LQG-2-51-2 (2)(AD,5\%,1.0,ee).lcd } \\ \text { Method Filename } & : \text { GWJ single.Icm } \\ \text { Batch Filename } & : \\ \text { Vial \# } & : 1-1 \\ \text { Injection Volume } & : 15 \text { uL } \\ \text { Date Acquired } & : 4 / 16 / 201910: 49: 41 \mathrm{PM} \\ \text { Date Processed } & : 4 / 16 / 201910: 57: 44 \mathrm{PM}\end{array}$

Sample Type : Unknown

Acquired by : : System Administrator

Processed by : System Administrator

\section{$<$ Chromatogram $>$}

$\mathrm{mV}$

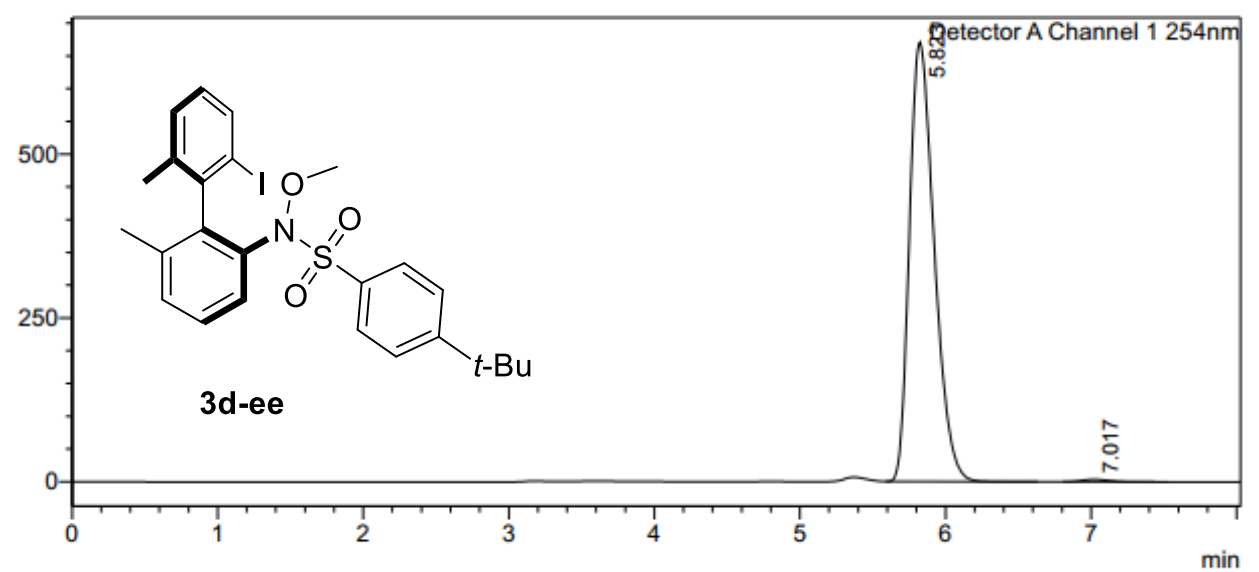

<Peak Table>

Detector A Channel $1254 \mathrm{~nm}$

\begin{tabular}{|r|r|r|r|r|r|r|r|}
\hline Peak\# & Ret. Time & \multicolumn{1}{|c|}{ Area } & \multicolumn{1}{c|}{ Height } & \multicolumn{1}{c|}{ Conc. } & Unit & Mark & Name \\
\hline 1 & 5.823 & 7953873 & 670757 & 99.422 & & $\mathrm{M}$ & \\
\hline 2 & 7.017 & 46271 & 3632 & 0.578 & & $\mathrm{M}$ & \\
\hline Total & & 8000144 & 674389 & & & & \\
\hline
\end{tabular}


Data File F: \DATA\LQG DATA\1qg-1-151-1(AD, 5X,1.6, RACE).D

Sample Name: 1qg-1-151-1(AD, 5\%, 1. $\theta$, RACE)
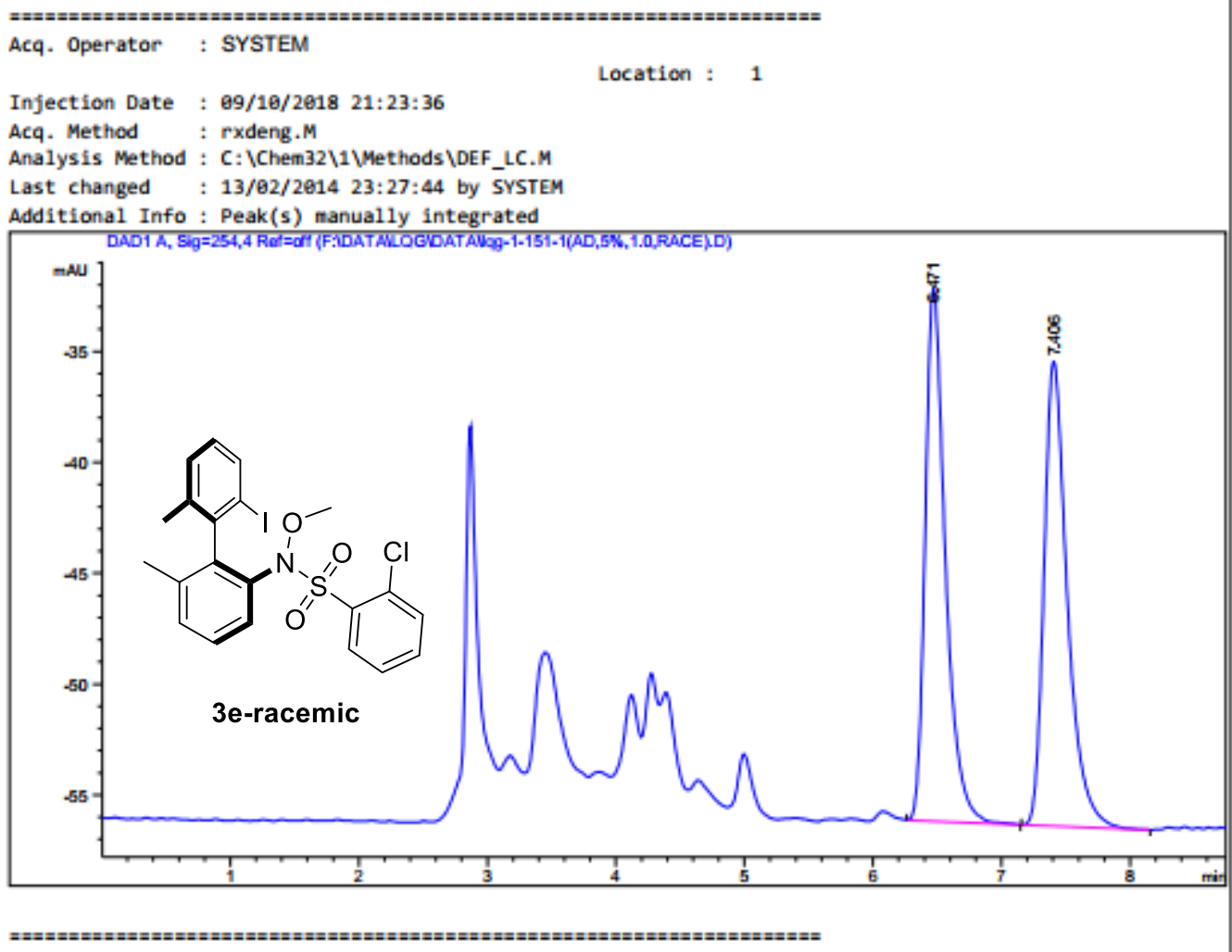

Area Percent Report

\begin{tabular}{|c|c|c|}
\hline Sorted By & : & Signal \\
\hline Multiplier & : & 1.0090 \\
\hline Dilution & : & 1.6980 \\
\hline
\end{tabular}

Use Multiplier \& Dilution Factor with ISTDs

Signal 1: DAD1 A, Sig $=254,4$ Ref $=0$ ff

\begin{tabular}{|c|c|c|c|c|c|}
\hline $\begin{array}{c}\text { Peak } \\
y\end{array}$ & $\begin{array}{l}\text { RetTime Type } \\
\text { [min] }\end{array}$ & $\begin{array}{l}\text { Width } \\
\text { [min] }\end{array}$ & $\begin{array}{c}\text { Area } \\
\text { [nAU*s] }\end{array}$ & $\begin{array}{l}\text { Height } \\
\text { [mall] }\end{array}$ & $\begin{array}{l}\text { Area } \\
\mathbf{x}\end{array}$ \\
\hline$\cdots$ & 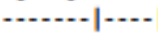 & (n) & (2) & (n) & . \\
\hline 1 & $6.471 \mathrm{BB}$ & 0.1635 & 262.93973 & 24.05271 & 49.8812 \\
\hline 2 & $7.466 \mathrm{BB}$ & 0.1891 & 264.19220 & 20.95713 & 50.1188 \\
\hline Total & & & 527.13193 & 45.00984 & \\
\hline
\end{tabular}

$* * *$ End of Report *** 
Data File F: \DATA\LQG DATAYLQG-2-56-3(AD, 5X,1.e,ee).D

Sample Name: LQG-2-56-3(AD,5X,1.0,ee)
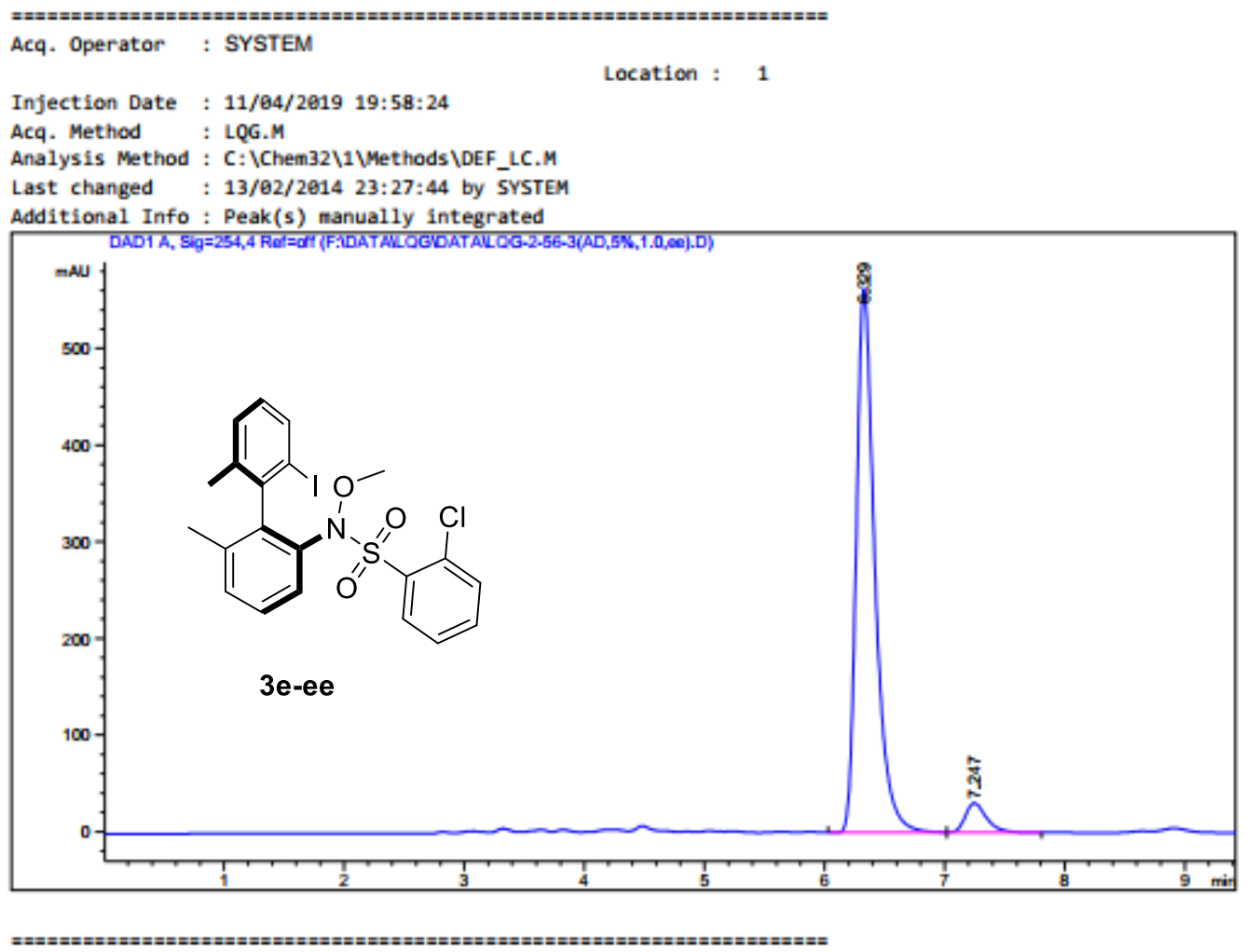

Area Percent Report

\begin{tabular}{|c|c|c|}
\hline Sorted By & : & Signal \\
\hline Multiplier & : & 1.0980 \\
\hline Dilution & : & 1.6980 \\
\hline
\end{tabular}

Use Multiplier \& Dilution Factor with ISTDS

Signal 1: DAD1 A, Sig $=254,4$ Ref $=$ off

\begin{tabular}{|c|c|c|c|c|c|}
\hline $\begin{array}{c}\text { Peak } \\
y\end{array}$ & $\begin{array}{l}\text { RetTime Type } \\
\text { [min] }\end{array}$ & $\begin{array}{l}\text { Width } \\
\text { [min] }\end{array}$ & $\begin{array}{c}\text { Area } \\
\text { [nAU*s] }\end{array}$ & $\begin{array}{l}\text { Height } \\
\text { [mall] }\end{array}$ & $\begin{array}{c}\text { Area } \\
\mathbf{x}\end{array}$ \\
\hline 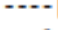 & - & & 1 & 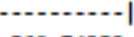 & (n........... \\
\hline 1 & $6.329 \mathrm{BV}$ & 0.1615 & 6054.99707 & 562.74353 & 94.1444 \\
\hline 2 & $7.247 \mathrm{VB}$ & 0.1859 & 376.68693 & 30.53773 & 5.8556 \\
\hline
\end{tabular}

Totals :

$6431.69490 \quad 593.28126$

$* * *$ End of Report **** 
<Sample Information>

Sample Name : LQG-2-99(AD-5\%, 1.0,race)

Sample ID

Data Filename : LQG-2-99(AD-5\%, 1.0, race).Icd

Method Filename : GWJ single.Icm

Batch Filename

Vial \#

$1-1$

Injection Volume : 15 uL

Date Acquired : 4/28/2019 3:58:10 PM

Date Processed $\quad: 4 / 28 / 2019$ 4:57:55 PM

Sample Type : Unknown

Acquired by $\quad$ : System Administrator

Processed by : : System Administrator

\section{$<$ Chromatogram >}

$\mathrm{mV}$

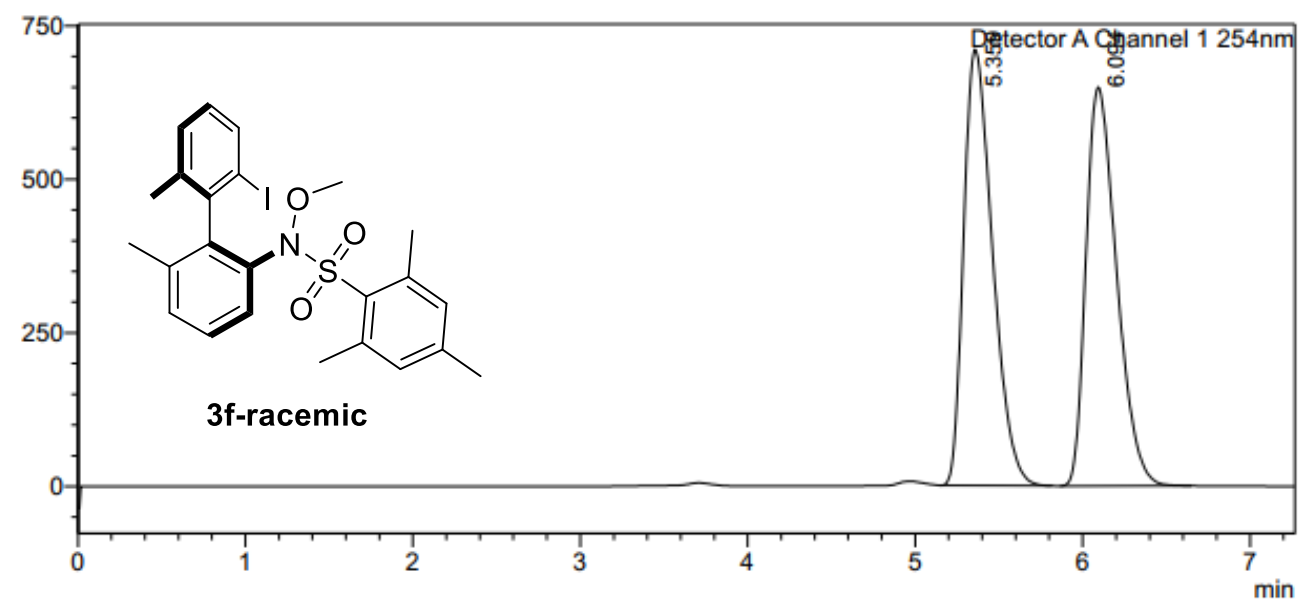

<Peak Table>

Detector A Channel $1254 \mathrm{~nm}$

\begin{tabular}{|r|r|r|r|r|r|r|r|}
\hline Peak\# & Ret. Time & \multicolumn{1}{|c|}{ Area } & \multicolumn{1}{c|}{ Height } & \multicolumn{1}{c|}{ Conc. } & Unit & Mark & Name \\
\hline 1 & 5.359 & 8514583 & 709437 & 50.763 & & $\mathrm{M}$ & \\
\hline 2 & 6.094 & 8258544 & 649237 & 49.237 & & $\mathrm{M}$ & \\
\hline Total & & 16773127 & 1358675 & & & & \\
\hline
\end{tabular}


<Sample Information>

Sample Name : LQG-2-99(AD-5\%, 1.0,ee)

Sample ID

LQG-2-99(AD-5\%, 1.0,ee) Icd

Data Filename
Method Filename : GWJ single.lcm

Batch Filename :

Vial \#

$1-1$

Date Acquired : : 4/28/2019 4:07:36 PM

Sample Type : Unknown

Acquired by : System Administrator Processed by : System Administrator

\section{$<$ Chromatogram $>$}

$\mathrm{mV}$

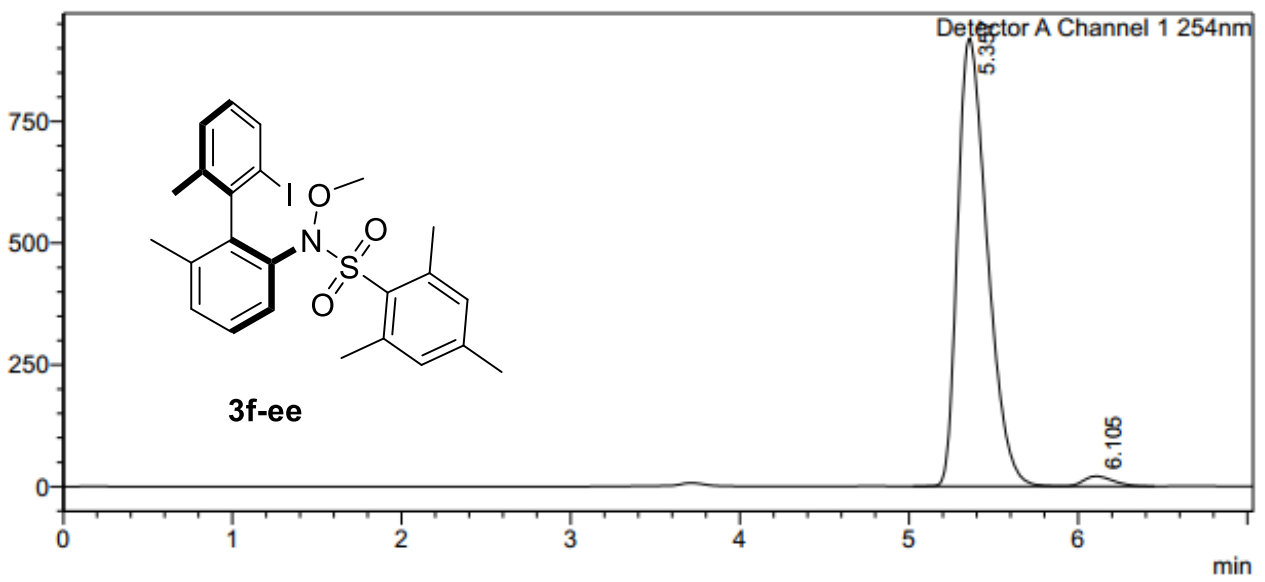

<Peak Table>

Detector A Channel $1254 \mathrm{~nm}$

\begin{tabular}{|r|r|r|r|r|r|r|r|}
\hline Peak\# & Ret. Time & \multicolumn{1}{c|}{ Area } & Height & \multicolumn{1}{c|}{ Conc. } & Unit & Mark & Name \\
\hline 1 & 5.357 & 11247920 & 920082 & 97.723 & & & \\
\hline 2 & 6.105 & 262117 & 20958 & 2.277 & & V & \\
\hline Total & & 11510037 & 941040 & & & & \\
\hline
\end{tabular}


Data File F: \DATAYLQG DATA\lqg-1-151-2(AD, 5X,1.6, RACE).D Sample Name: 1qg-1-151-2(AD, 58,1.0, RACE)
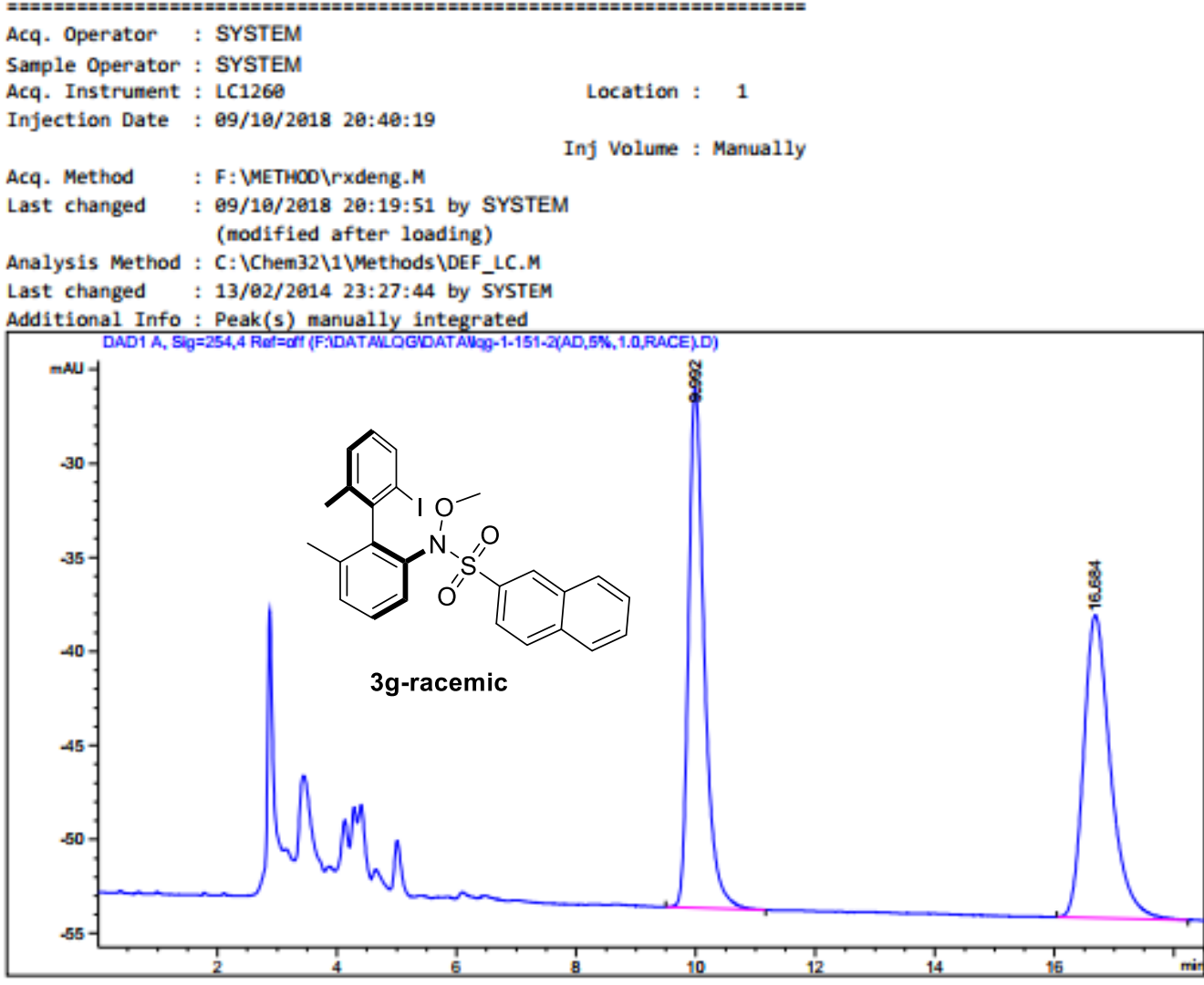

Area Percent Report

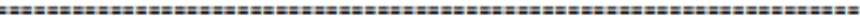

$\begin{array}{lll}\text { Sorted By } & : & \text { Signal } \\ \text { Multiplier } & : & 1.0999\end{array}$

1.9989

Use Multiplier \& Dilution Factor with ISTDs

Signal 1: DAD1 A, Sig $=254,4$ Ref $=0$ ff

\begin{tabular}{cccccc}
$\begin{array}{c}\text { Peak RetTime Type width } \\
\text { [min] }\end{array}$ & $\begin{array}{c}\text { Area } \\
\text { [min] }\end{array}$ [nAU*s] & $\begin{array}{l}\text { Height } \\
\text { [mall }\end{array}$ & $\begin{array}{c}\text { Area } \\
\mathbf{x}\end{array}$ \\
\hline 1 & $9.992 \mathrm{BB}$ & 0.2773 & 506.69492 & 27.71560 & 50.2195 \\
\hline 2 & $16.684 \mathrm{BB}$ & 0.4739 & 502.26578 & 16.10133 & 49.7865
\end{tabular}

Totals :

$1098.96969 \quad 43.81694$ 
Data File F: \DATAYLQG DATAYlqg-3-17(AD, 5X,1.6, EE).D

Sample Name: 1qg-3-17(AD, 5X,1.6, EE)

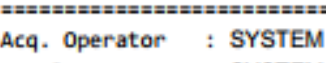

Sample Operator : SYSTEM

Acq. Instrument : LC1268

Injection Date : 16/06/2019 09:40:37

Acq. Method : F: METHCOYLQG.MILG.M

Location : 1

Inj Volume : No inj

Last changed : 16/06/2019 09:37:20 by SYSTEM

(modified after loading)

Analysis Method : C: \Chem32\1\Methods \DEF_LC.M

Last changed : 13/02/2814 $23: 27: 44$ by SYSTEM

Additional Info: Peak(s) manually integrated

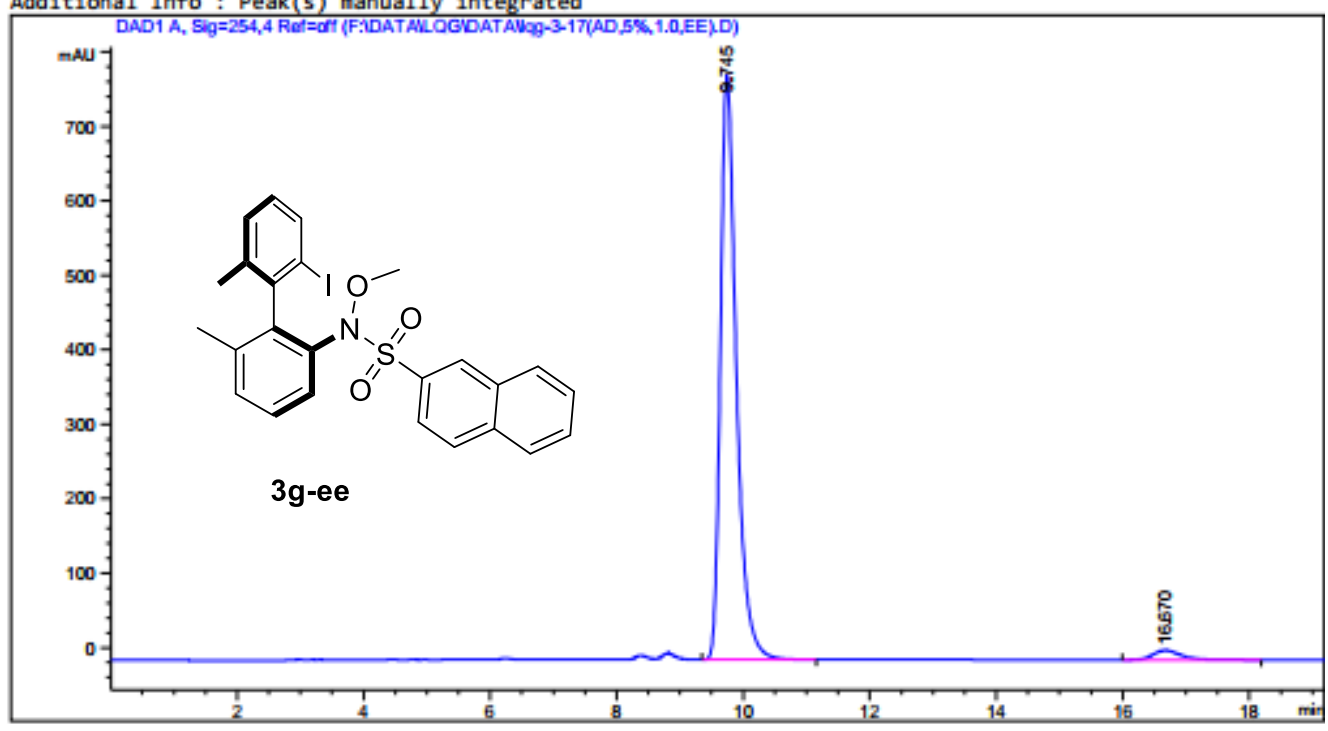

Area Percent Report

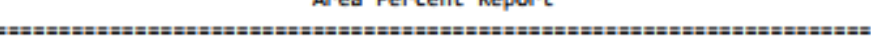

Sorted By : Signal

Multiplier : 1.8090

Dilution : 1.0980

Use Multiplier \& Dilution Factor with ISTDS

Signal 1: DAD1 A, Sig $=254,4$ Ref $=0$ ff

\begin{tabular}{|c|c|c|c|c|c|}
\hline $\begin{array}{c}\text { Peak } \\
y\end{array}$ & $\begin{array}{l}\text { RetTime Type } \\
\text { [min] }\end{array}$ & $\begin{array}{l}\text { Width } \\
\text { [min] }\end{array}$ & $\begin{array}{c}\text { Area } \\
\text { [nAU*s] }\end{array}$ & $\begin{array}{l}\text { Height } \\
\text { [maU]] }\end{array}$ & $\begin{array}{l}\text { Area } \\
\mathbf{x}\end{array}$ \\
\hline$\cdots$ & 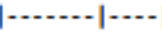 & $\ldots$ & | & - & | \\
\hline 1 & $9.745 \mathrm{BB}$ & 0.2632 & $1.36613 \mathrm{e} 4$ & 784.35779 & 97.2126 \\
\hline 2 & $16.670 \mathrm{BB}$ & 0.4599 & 391.71805 & 12.76794 & 2.7874 \\
\hline
\end{tabular}

Totals :

$1.46536 \mathrm{e} 4797.12573$ 
Data File F: \DATAYLQG DATAYLQG-2-56-1(2)(AD, 58, 1.0, race).D Sample Name: LQQG-2-56-1(2) (AD, 1ex, 1.0, race)
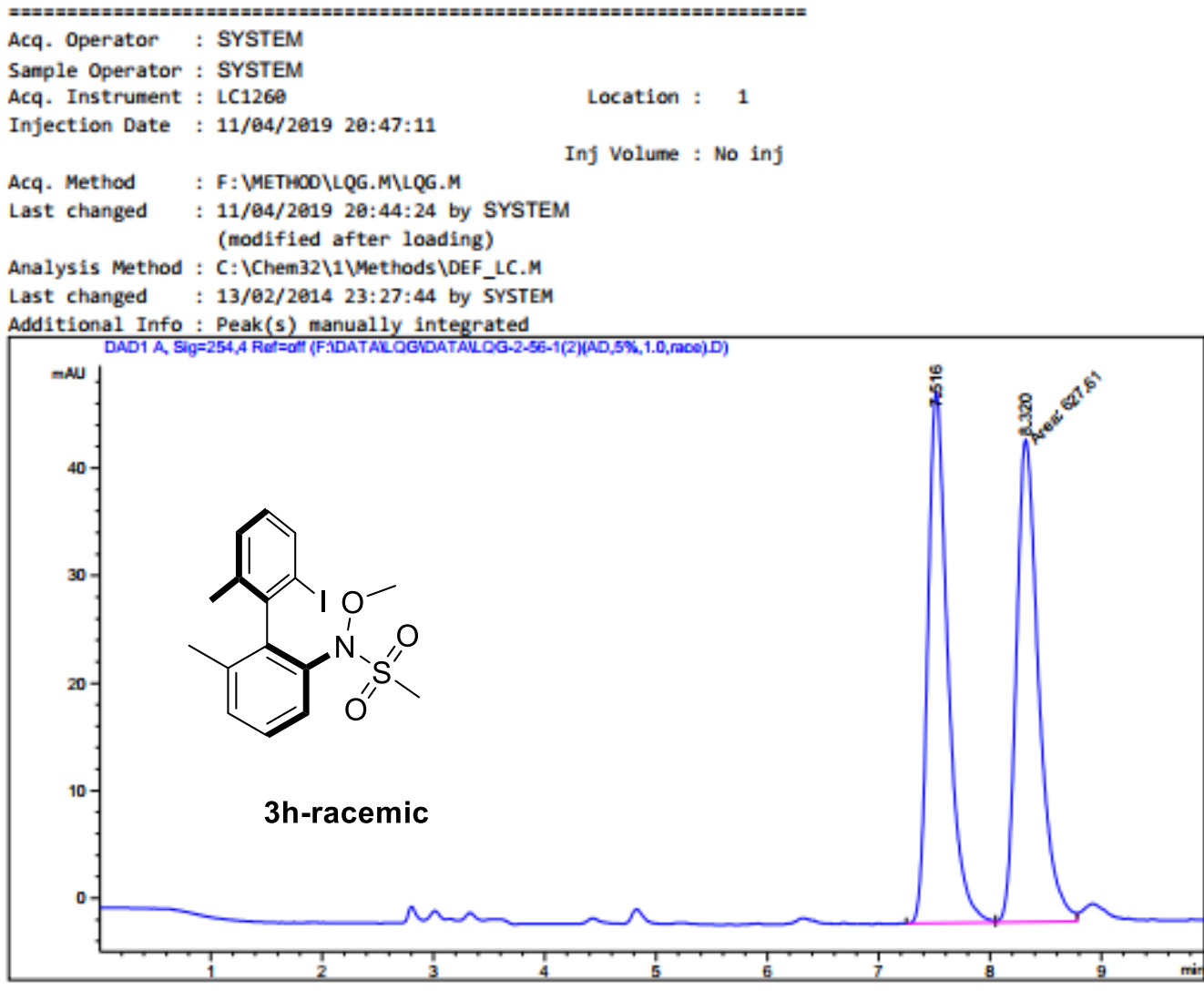

Area Percent Report 
Data File F: \DATAYLQG DATAYLQG-2-56-1(AD, 5X,1.e, ee).D Sample Name: LQG-2-56-1(AD, 5X,1.0, ee)

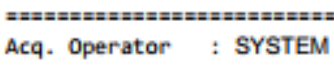

Injection Date : 11/64/2819 20:59:46

Acq. Method : LQG.M

Analysis Method : C: \Chem32\1 Methods \DEF LC.M

Last changed : 13/02/2814 23:27:44 by SYSTEM

Additional Info : Peak(s) manually integrated

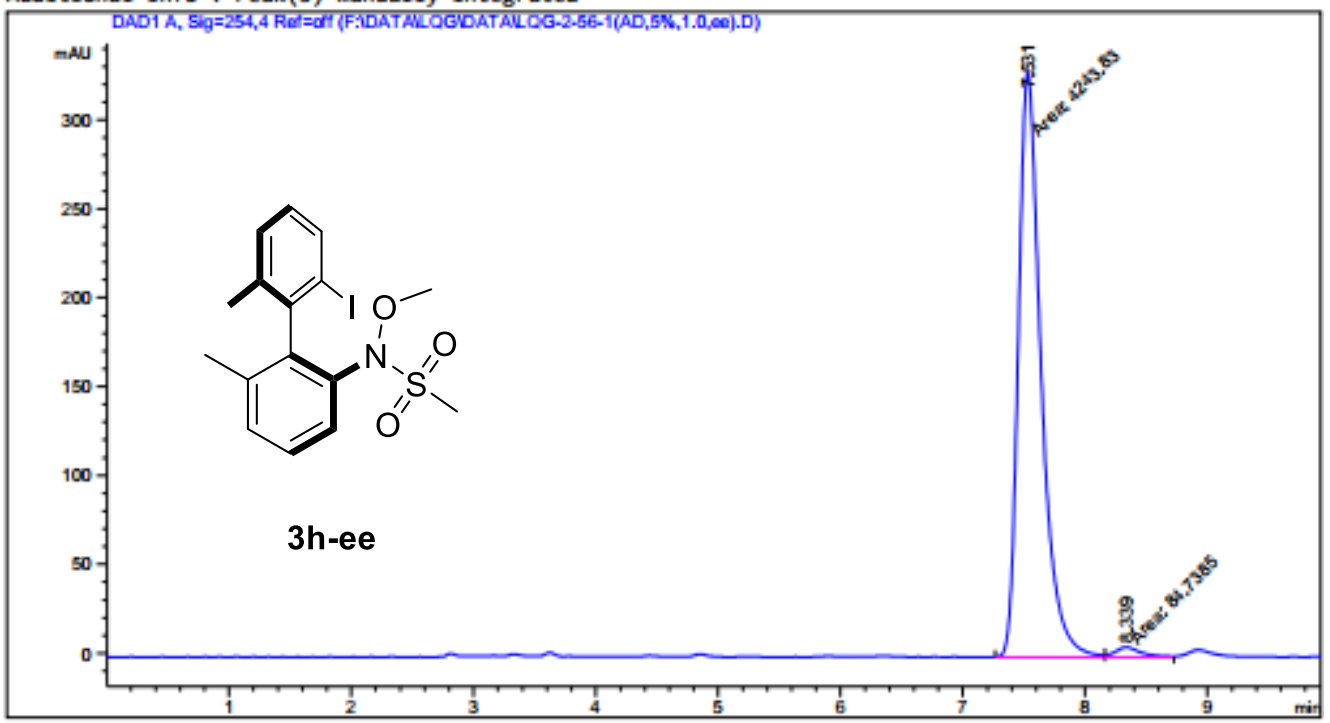

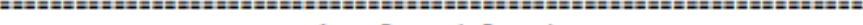

Area Percent Report

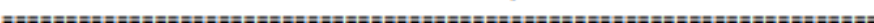

$\begin{array}{lll}\text { Sorted By } & : & \text { Signal } \\ \text { Multiplier } & : & 1.6980\end{array}$

Dilution : 1.0909

Use Multiplier \& Dilution Factor with ISTDS

Signal 1: DAD1 A, Sig $=254,4$ Ref $=0$ ff

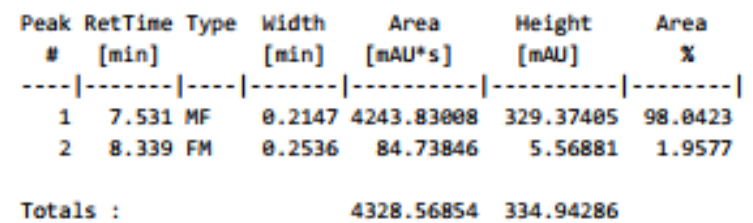

$* * *$ End of Report *** 
Data File F: \DATAYLQG DATA\lqg-2-50(AD, 10X,1.8, race).D

Sample Name: $1 \mathrm{qg}-2-50(A D, 10 \mathrm{x}, 1.6$, race)

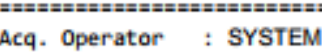

Sample Operator : SYSTEM

Acq. Instrument : LC1268

Injection Date : $07 / 04 / 2819$ 19:25:37

Location : 1

Acq. Method : F: METHOOYLQG.MILQG.M

Inj Volume : No inj

Last changed : $07 / 04 / 2019$ 19:17:58 by SYSTEM

(modified after loading)

Analysis Method : C: \Chem32\1\Methods \DEF_LC.M

Last changed : 13/02/2814 $23: 27: 44$ by SYSTEM

Additional Info: Peak(s) manually integrated

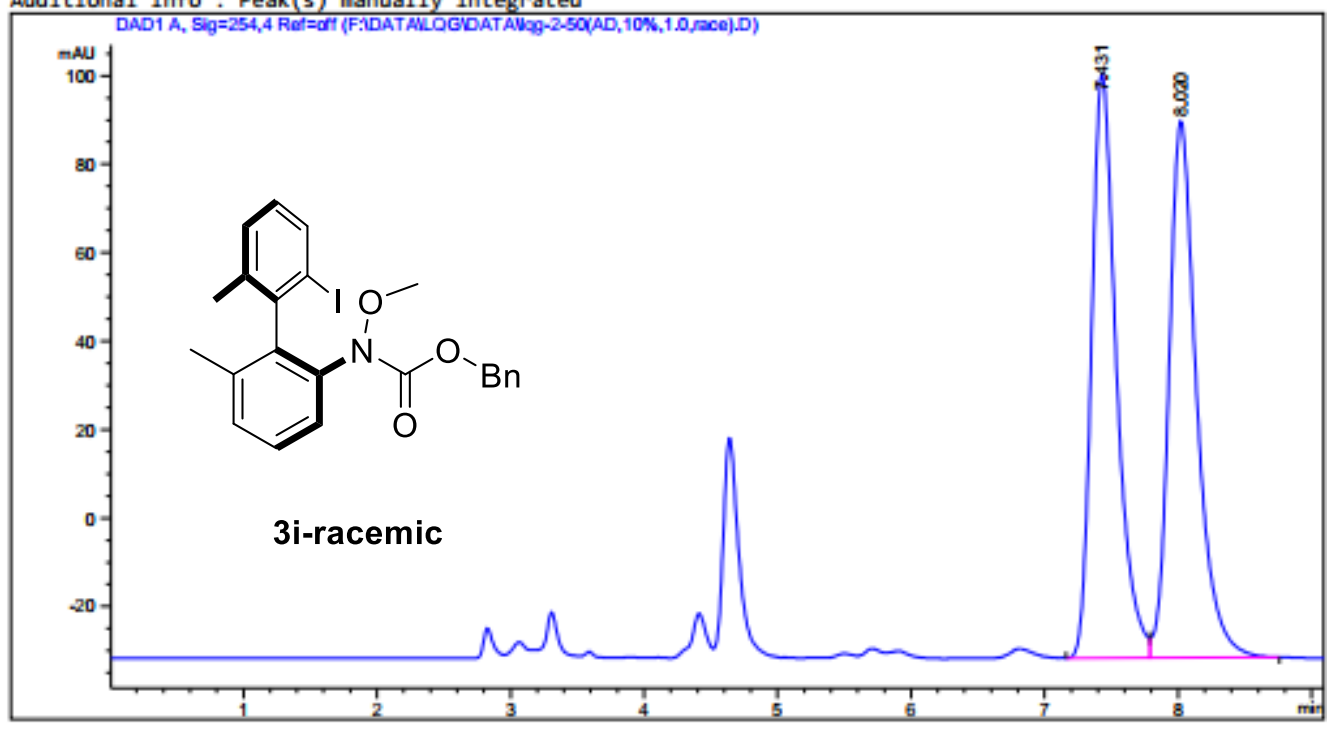

Area Percent Report

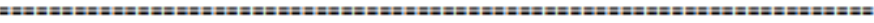

Sorted By : : Signal

Multiplier : : 1.6800

Dilution 1.8996

Use Multiplier \& Dilution Factor with ISTDS

Signal 1: DAD1 A, Sig $=254,4$ Ref $=$ off

\begin{tabular}{|c|c|c|c|c|c|}
\hline $\begin{array}{c}\text { Peak } \\
y\end{array}$ & $\begin{array}{l}\text { RetTime Type } \\
\text { [min] }\end{array}$ & $\begin{array}{l}\text { Width } \\
\text { [min] }\end{array}$ & $\begin{array}{c}\text { Area } \\
\text { [nAU*s] }\end{array}$ & $\begin{array}{l}\text { Height } \\
\text { [mau]] }\end{array}$ & $\begin{array}{l}\text { Area } \\
\mathbf{x}\end{array}$ \\
\hline & & & 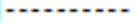 & (n) & $\cdots$ \\
\hline 1 & 7.431 BV & 0.1967 & 1705.54810 & 132.07222 & 49.5472 \\
\hline 2 & 8.020 VB & 0.2172 & 1736.72870 & 121.21343 & 50.4528 \\
\hline
\end{tabular}

Totals :

$3442.26889 \quad 253.28565$ 
Data File F: \DATA\LQG DATA\lag-2-50(AD, 10X,1.6, ee).D

Sample Name: 1qg-2-50(AD, 18x,1.6, ee)
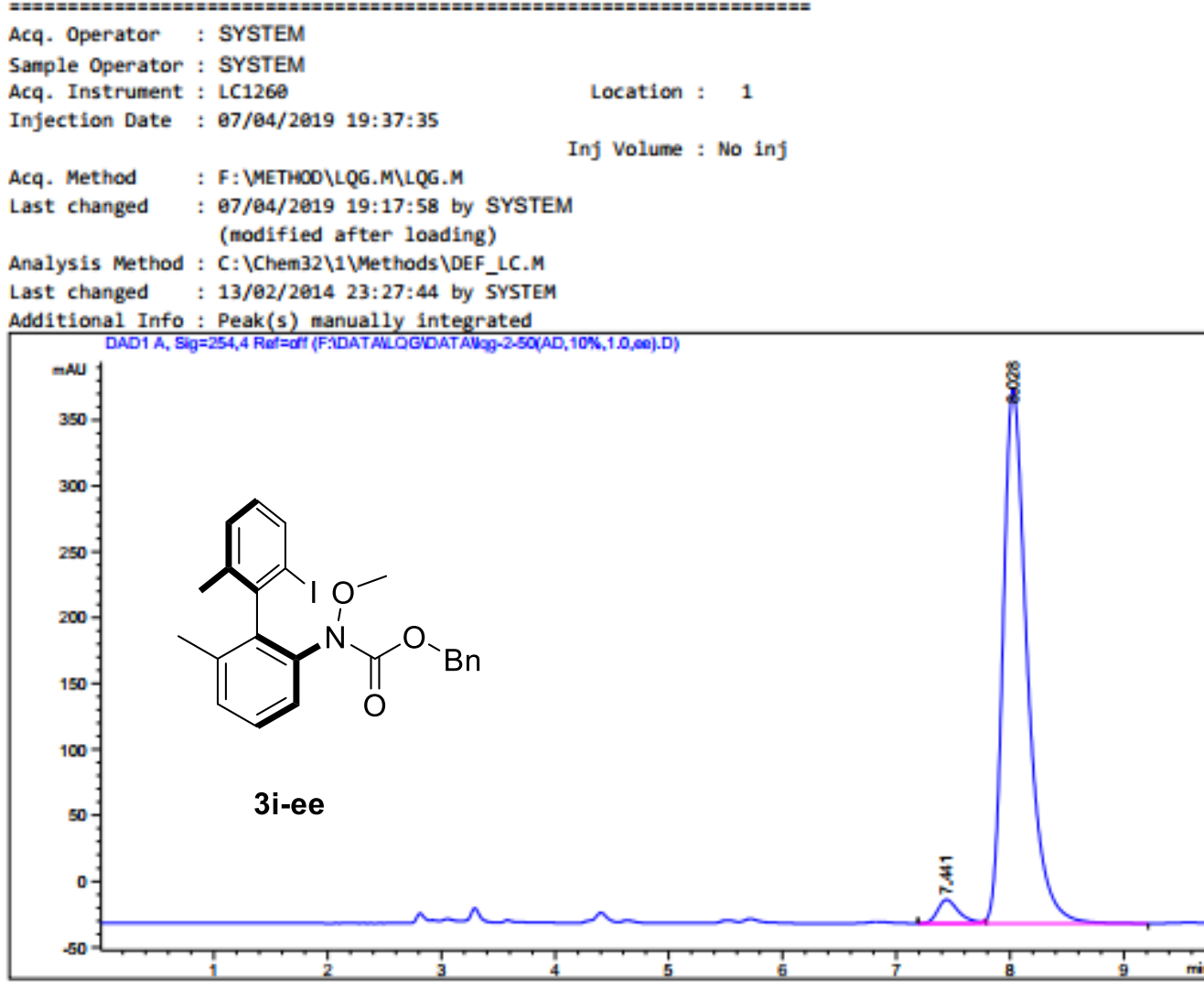

Area Percent Report

\begin{tabular}{|c|c|c|}
\hline Sorted By & : & Signal \\
\hline Multiplier & : & 1.0990 \\
\hline Dilution & : & 1.0980 \\
\hline
\end{tabular}

Use Multiplier \& Dilution Factor with ISTDS

Signal 1: DAD1 A, Sig $=254,4$ Ref $=0$ ff

\begin{tabular}{|c|c|c|c|c|c|}
\hline $\begin{array}{c}\text { Peak } \\
y\end{array}$ & $\begin{array}{l}\text { RetTime Type } \\
\text { [min] }\end{array}$ & $\begin{array}{l}\text { Width } \\
\text { [min] }\end{array}$ & $\begin{array}{c}\text { Area } \\
\text { [nAU*s] }\end{array}$ & $\begin{array}{l}\text { Height } \\
\text { [mau] }\end{array}$ & $\begin{array}{l}\text { Area } \\
\mathbf{x}\end{array}$ \\
\hline 2 & 10 & - & | & & \\
\hline 1 & 7.441 BV E & 0.1919 & 229.95912 & 17.89172 & 3.6998 \\
\hline 2 & 8.028 VB R & 0.2239 & 5985.53516 & 486.23102 & 96.3002 \\
\hline
\end{tabular}

Totals :

$6215.49428 \quad 424.12273$ 
Data File F: \DATAYLQG DATAYLQG-3-10(00,6.5X,1.6,RAC)-2.D

Sample Name: LQG-3-10(00,0.5X,1.0,RAC)-2

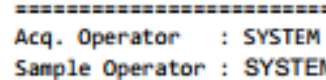

Acq. Instrument : LC126e

Injection Date : 13/66/2019 21:05:48

Acq. Method : F: METHOOYLQG.MYLG.M

Last changed : 13/06/2019 20:40:33 by SYSTEM

(modified after loading)

Analysis Method : C: \Chem32\1 Methods \DEF_LC.M

Last changed : 13/02/2014 23:27:44 by SYSTEM

Additional Info : Peak(s) manually integrated

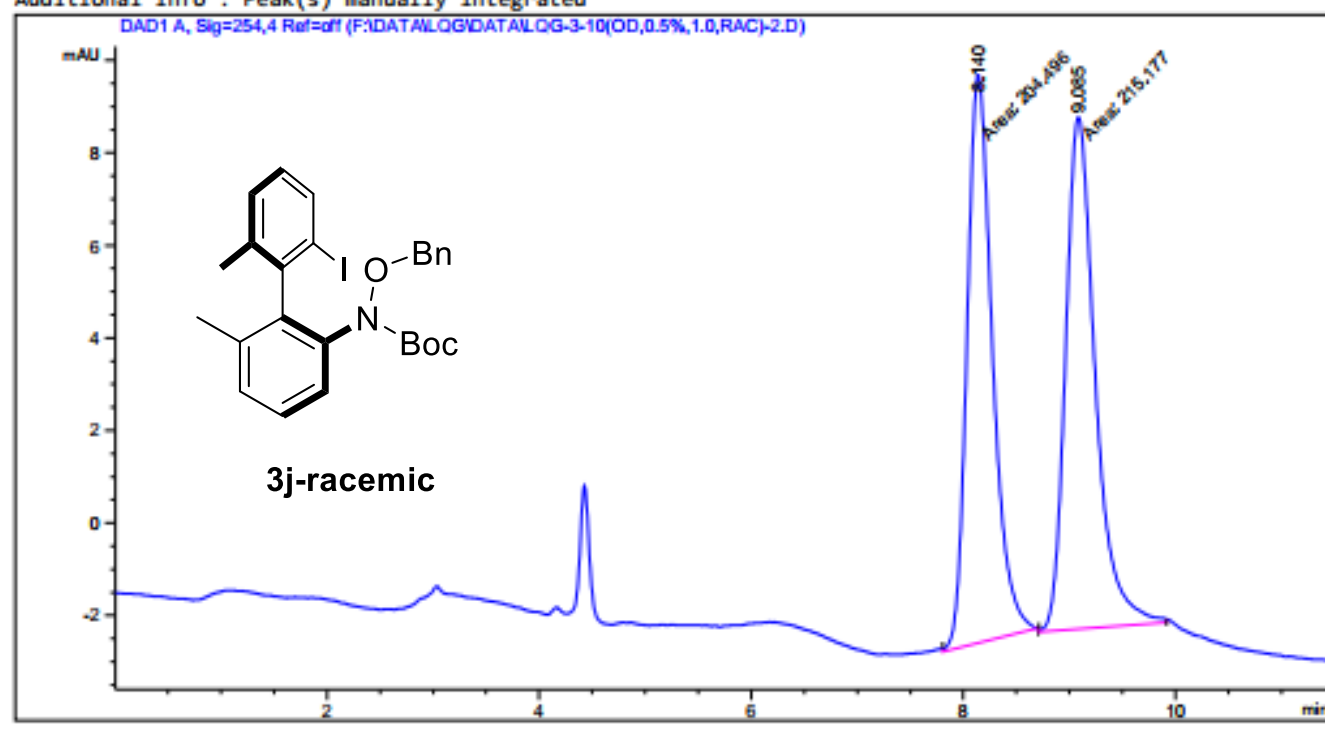

Area Percent Report

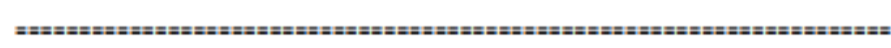

\begin{tabular}{|c|c|c|}
\hline Sorted By & : & Signal \\
\hline Multiplier & : & 1.6090 \\
\hline Dilution & : & 1.6900 \\
\hline
\end{tabular}

Use Multiplier \& Dilution Factor with ISTDS

Signal 1: DAD1 A, Sig $=254,4$ Ref $=0$ ff

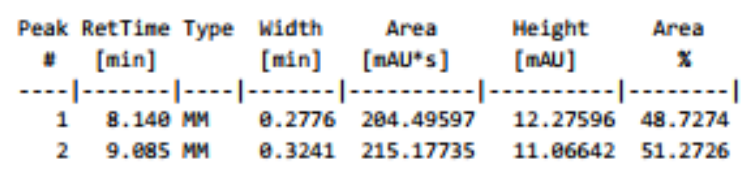

Totals :

$419.67332 \quad 23.34238$ 
Data File F: \DATAYLQG DATAYLQG-3-10( $\infty 0,0.5 X, 1.6, E E) . D$

Sample Name: LQG-3-10(00,0.5X,1.0,EE)
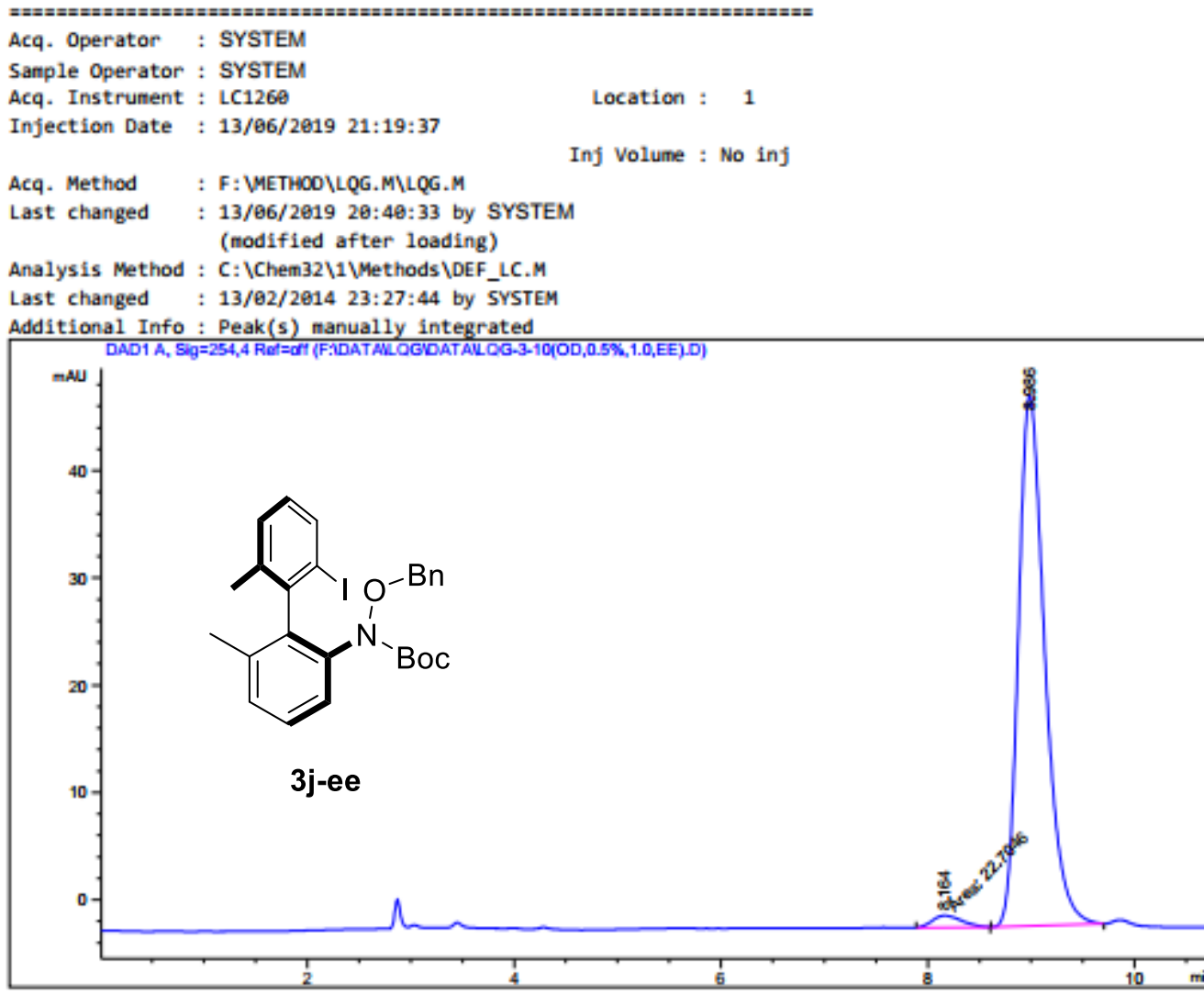

Area Percent Report

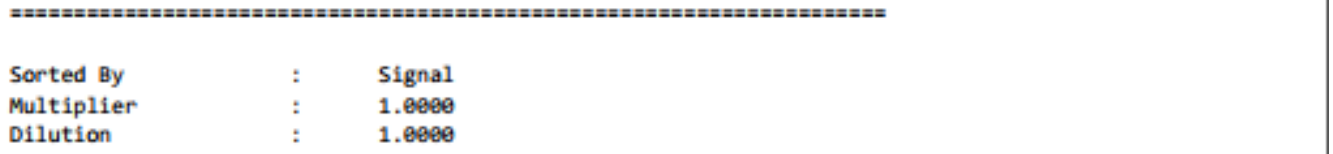

Use Multiplier \& Dilution Factor with ISTDS

Signal 1: DAD1 A, Sig $=254,4$ Ref $=$ off

\begin{tabular}{|c|c|c|c|c|c|}
\hline $\begin{array}{c}\text { Peak } \\
y\end{array}$ & $\begin{array}{l}\text { RetTime Type } \\
\text { [min] }\end{array}$ & $\begin{array}{l}\text { Width } \\
\text { [min] }\end{array}$ & $\begin{array}{c}\text { Area } \\
\text { [mAU*s] }\end{array}$ & $\begin{array}{l}\text { Height } \\
\text { [maU]] }\end{array}$ & $\begin{array}{l}\text { Area } \\
\mathbf{x}\end{array}$ \\
\hline & & - & |-1 & - & 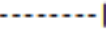 \\
\hline 1 & $8.164 \mathrm{MM}$ & 0.3359 & 22.78460 & 1.12672 & 2.5209 \\
\hline 2 & 8.986 BB & 0.2731 & 877.96289 & 49.46983 & 97.4791 \\
\hline
\end{tabular}

Totals

$900.66749 \quad 50.59655$ 


\section{<Sample Information>}

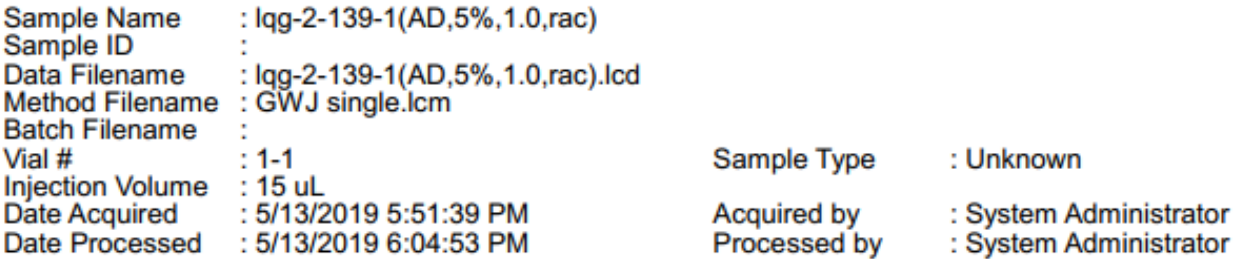

\section{$<$ Chromatogram $>$}

$\mathrm{mV}$

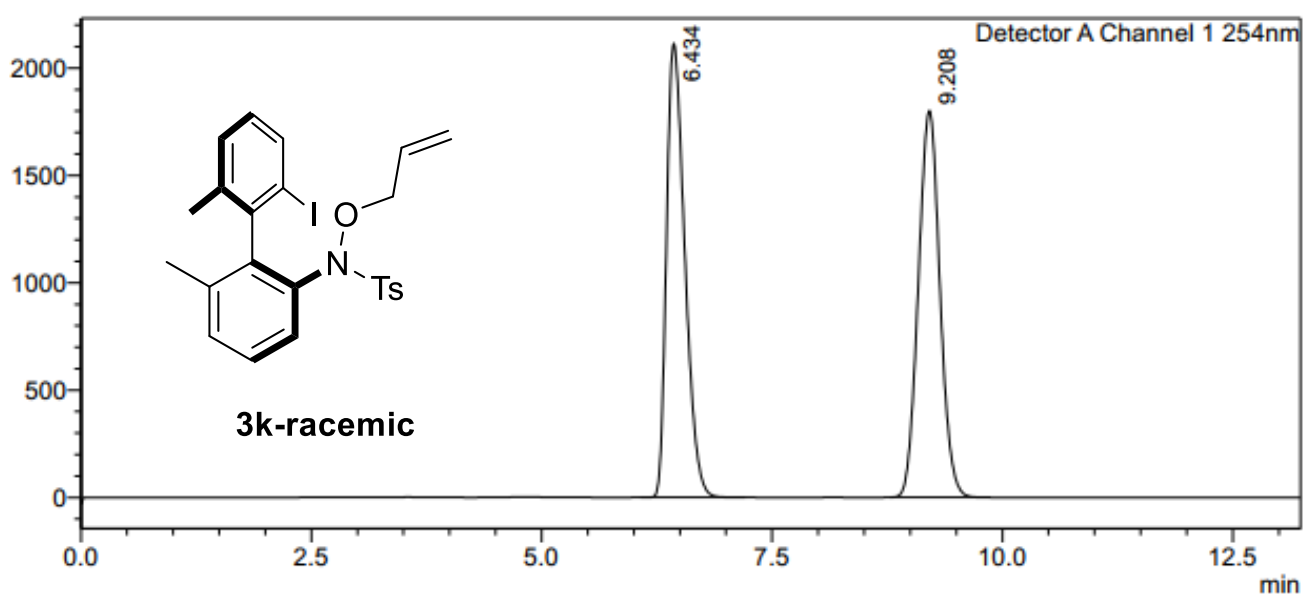

\section{<Peak Table>}

Detector A Channel $1254 \mathrm{~nm}$

\begin{tabular}{|r|r|r|r|r|r|r|r|}
\hline Peak\# & Ret. Time & \multicolumn{1}{c|}{ Area } & Height & \multicolumn{1}{c|}{ Conc. } & Unit & Mark & Name \\
\hline 1 & 6.434 & 29153697 & 2111293 & 49.815 & & $\mathrm{M}$ & \\
\hline 2 & 9.208 & 29369842 & 1800452 & 50.185 & & $\mathrm{M}$ & \\
\hline Total & & 58523539 & 3911745 & & & & \\
\hline
\end{tabular}




\section{<Sample Information>}

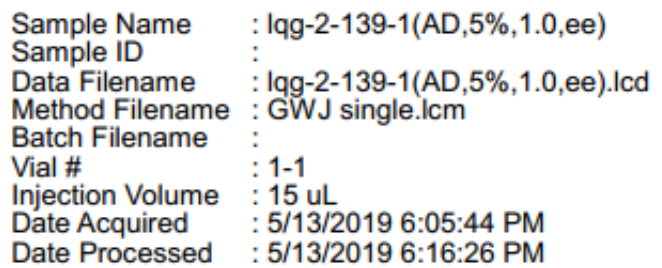

Acquired by : : System Administrator Processed by : : System Administrator

\section{$<$ Chromatogram>}

$\mathrm{mV}$

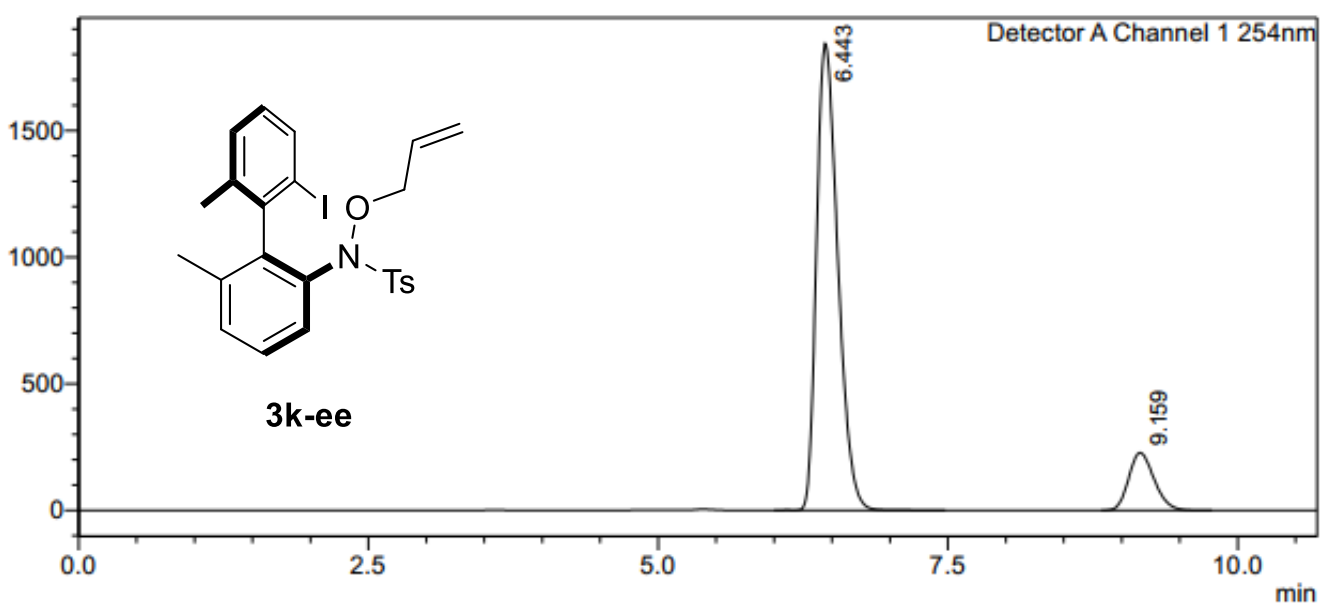

<Peak Table>

Detector A Channel $1254 \mathrm{~nm}$

\begin{tabular}{|r|r|r|r|r|r|r|r|}
\hline Peak\# & Ret. Time & \multicolumn{1}{c|}{ Area } & \multicolumn{1}{c|}{ Height } & Conc. & Unit & Mark & Name \\
\hline 1 & 6.443 & 23576801 & 1841594 & 87.145 & & $\mathrm{M}$ & \\
\hline 2 & 9.159 & 3477787 & 227148 & 12.855 & & $\mathrm{M}$ & \\
\hline Total & & 27054588 & 2068742 & & & & \\
\hline
\end{tabular}


<Sample Information>

Sample Name : LQG-2-61(AD,5\%,1.0,race)

Sample ID

Data Filename : LQG-2-61(AD,5\%,1.0,race).Icd

Method Filename : GWJ single.Icm

Batch Filename :

Vial \# : :1-1

Injection Volume : 15

Date Acquired : : 4/13/2019 6:48:46 PM

Sample Type : Unknown

Date Processed $\quad: 4 / 13 / 2019$ 7:01:36 PM

Acquired by : : System Administrator

Processed by : : System Administrator

\section{<Chromatogram>}

$\mathrm{mV}$

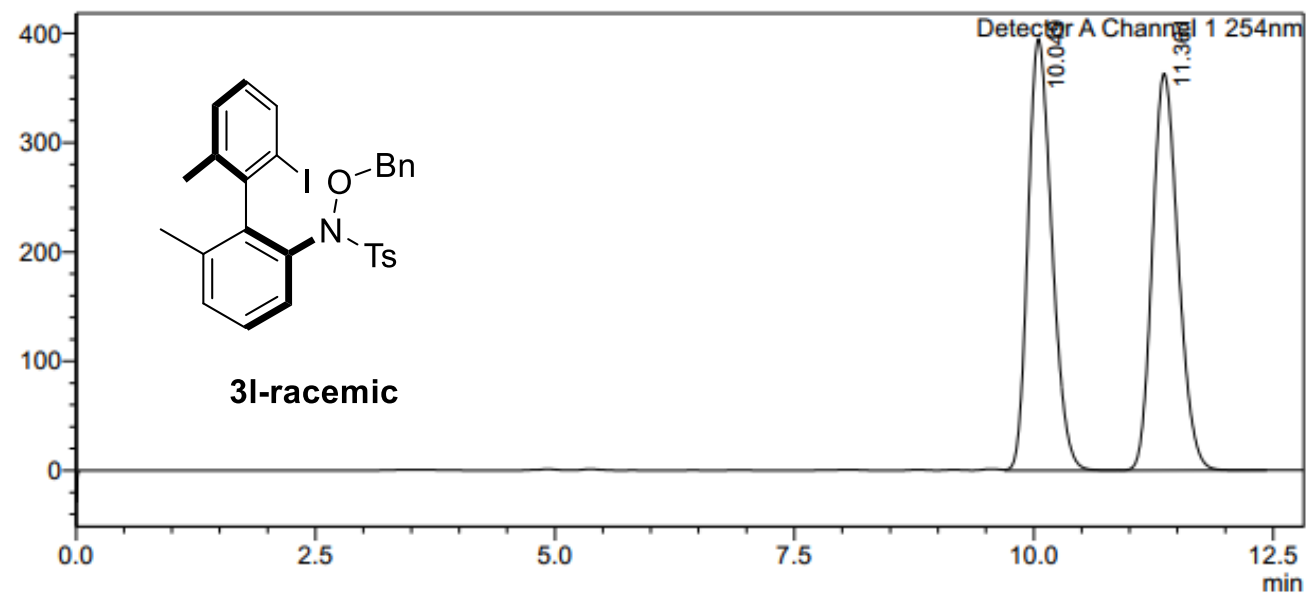

<Peak Table>

Detector A Channel $1254 \mathrm{~nm}$

\begin{tabular}{|r|r|r|r|r|r|r|r|}
\hline Peak\# & Ret. Time & \multicolumn{1}{c|}{ Area } & Height & Conc. & Unit & Mark & Name \\
\hline 1 & 10.049 & 6833796 & 394522 & 50.048 & & & \\
\hline 2 & 11.361 & 6820697 & 362831 & 49.952 & & V & \\
\hline Total & & 13654494 & 757353 & & & & \\
\hline
\end{tabular}


<Sample Information>

Sample Name : LQG-2-61(AD,5\%,1.0,ee)

Sample ID

Data Filename : LQG-2-61(AD,5\%,1.0,ee).Icd

Method Filename : GWJ single.Icm

Batch Filename :

Vial \# : : $1-1$

Injection Volume : $15 \mathrm{uL}$

Date Acquired : : 4/13/2019 7:06:26 PM

Sample Type : Unknown

Date Processed : : 4/13/2019 7:19:16 PM

Acquired by : System Administrator Processed by : System Administrator

\section{$<$ Chromatogram >}

$\mathrm{mV}$

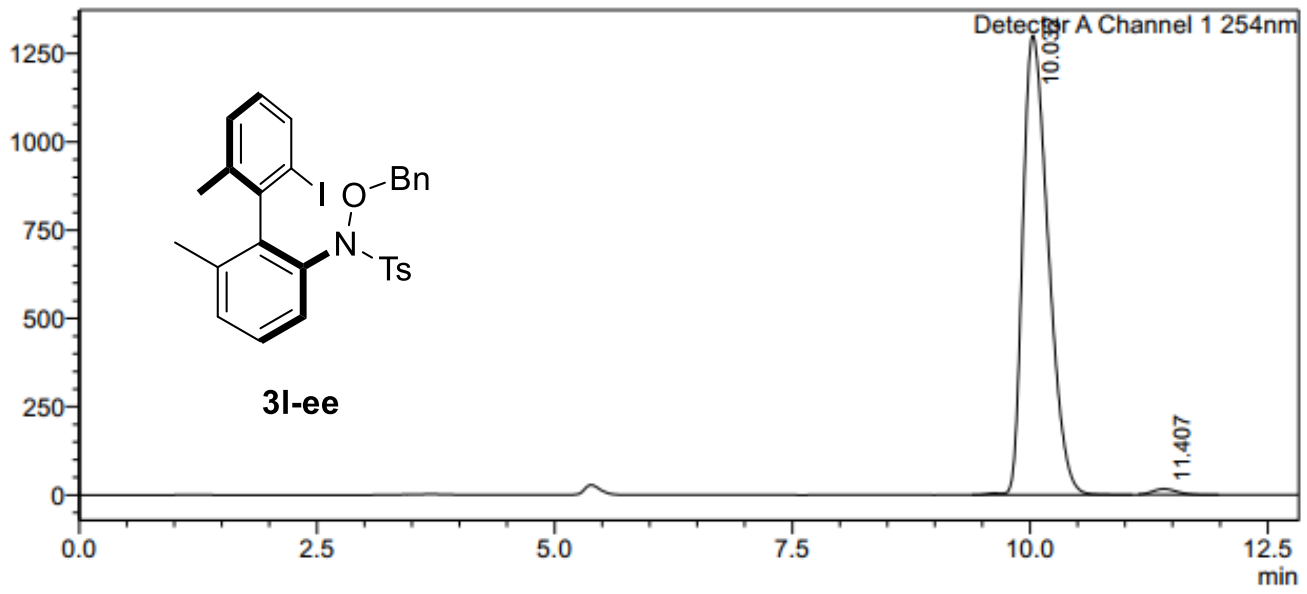

<Peak Table>

Detector A Channel $1254 \mathrm{~nm}$

\begin{tabular}{|r|r|r|r|r|r|r|r|}
\hline Peak\# & Ret. Time & \multicolumn{1}{|c|}{ Area } & \multicolumn{1}{c|}{ Height } & \multicolumn{1}{c|}{ Conc. } & Unit & Mark & Name \\
\hline 1 & 10.032 & 24222313 & 1300280 & 98.829 & & $\mathrm{M}$ & \\
\hline 2 & 11.407 & 286886 & 16385 & 1.171 & & $\mathrm{M}$ & \\
\hline Total & & 24509199 & 1316664 & & & & \\
\hline
\end{tabular}


<Sample Information>

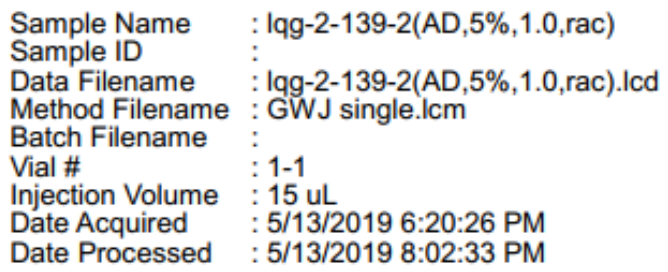

Sample Type : Unknown

Acquired by : System Administrator Processed by : System Administrator

\section{$<$ Chromatogram>}

$\mathrm{mV}$

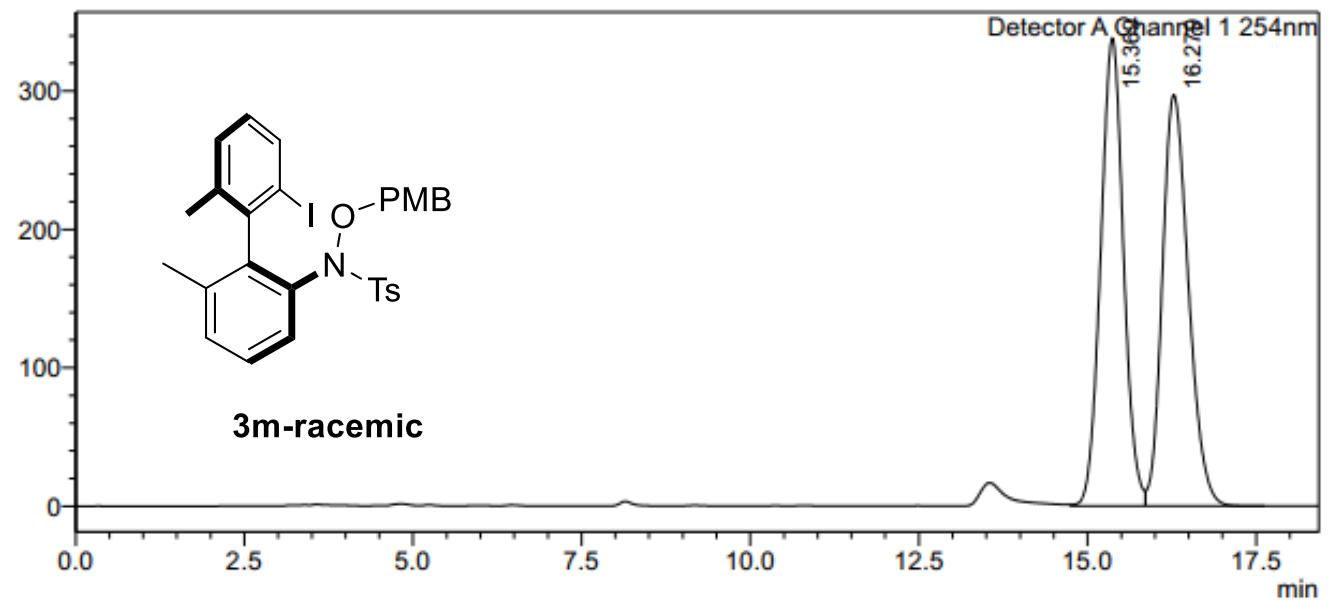

<Peak Table>

Detector A Channel $1254 \mathrm{~nm}$

\begin{tabular}{|r|r|r|r|r|r|r|r|}
\hline Peak\# & Ret. Time & \multicolumn{1}{c|}{ Area } & Height & Conc. & Unit & Mark & Name \\
\hline 1 & 15.369 & 7962296 & 337984 & 49.907 & & & \\
\hline 2 & 16.279 & 7992077 & 297179 & 50.093 & & SV & \\
\hline Total & & 15954373 & 635163 & & & & \\
\hline
\end{tabular}


<Sample Information>

Sample Name $\quad$ : lqg-2-139-2(AD,5\%,1.0,ee)

Sample ID

Data Filename : : lqg-2-139-2(AD,5\%,1.0,ee).lcd

Method Filename : GWJ single.lcm

Batch Filename

Vial \# : :1-1

Injection Volume : $15 \mathrm{uL}$

Date Acquired $\quad: 5 / 13 / 2019$ 6:47:05 PM

Date Processed : :5/13/2019 8:01:14 PM

Sample Type : : Unknown

Acquired by : : System Administrator Processed by : : System Administrator

\section{$<$ Chromatogram>}

$\mathrm{mV}$

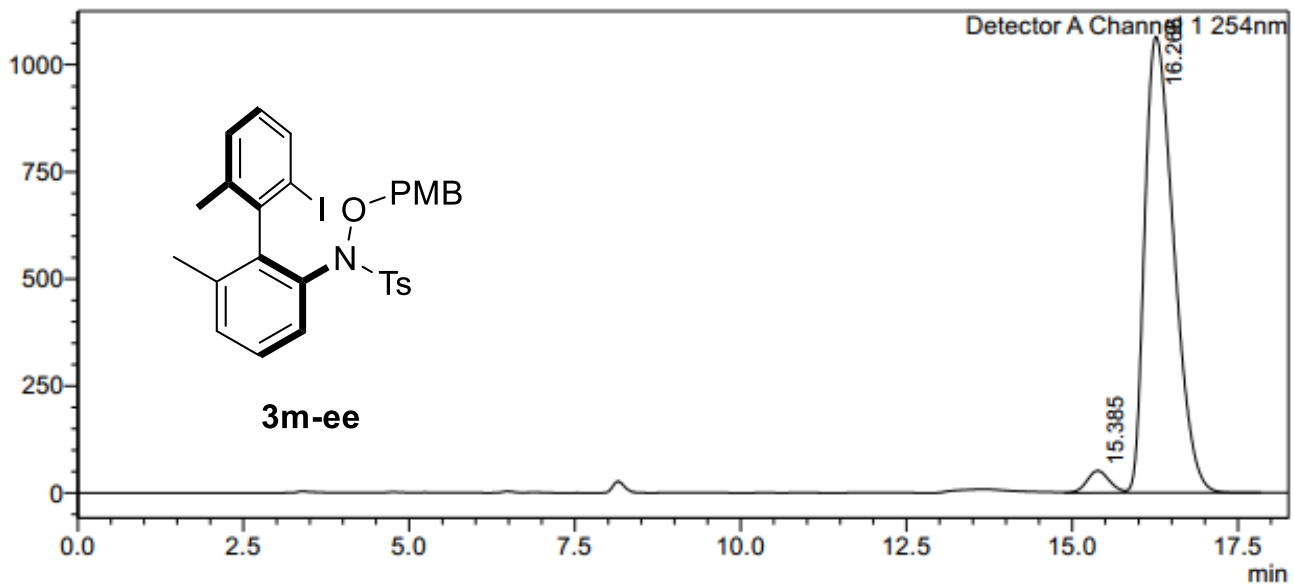

<Peak Table>

Detector A Channel $1254 \mathrm{~nm}$

\begin{tabular}{|r|r|r|r|r|r|r|r|}
\hline Peak\# & Ret. Time & \multicolumn{1}{c|}{ Area } & Height & Conc. & Unit & Mark & Name \\
\hline 1 & 15.385 & 1220020 & 51682 & 3.621 & & & \\
\hline 2 & 16.266 & 32468845 & 1065603 & 96.379 & & V & \\
\hline Total & & 33688864 & 1117286 & & & & \\
\hline
\end{tabular}


Data File F: \DATAYLQG DATAYLQG-1-179(AD, 5X,1.e, race).D Sample Name: LQG-1-179(AD, 5X,1.6, race)
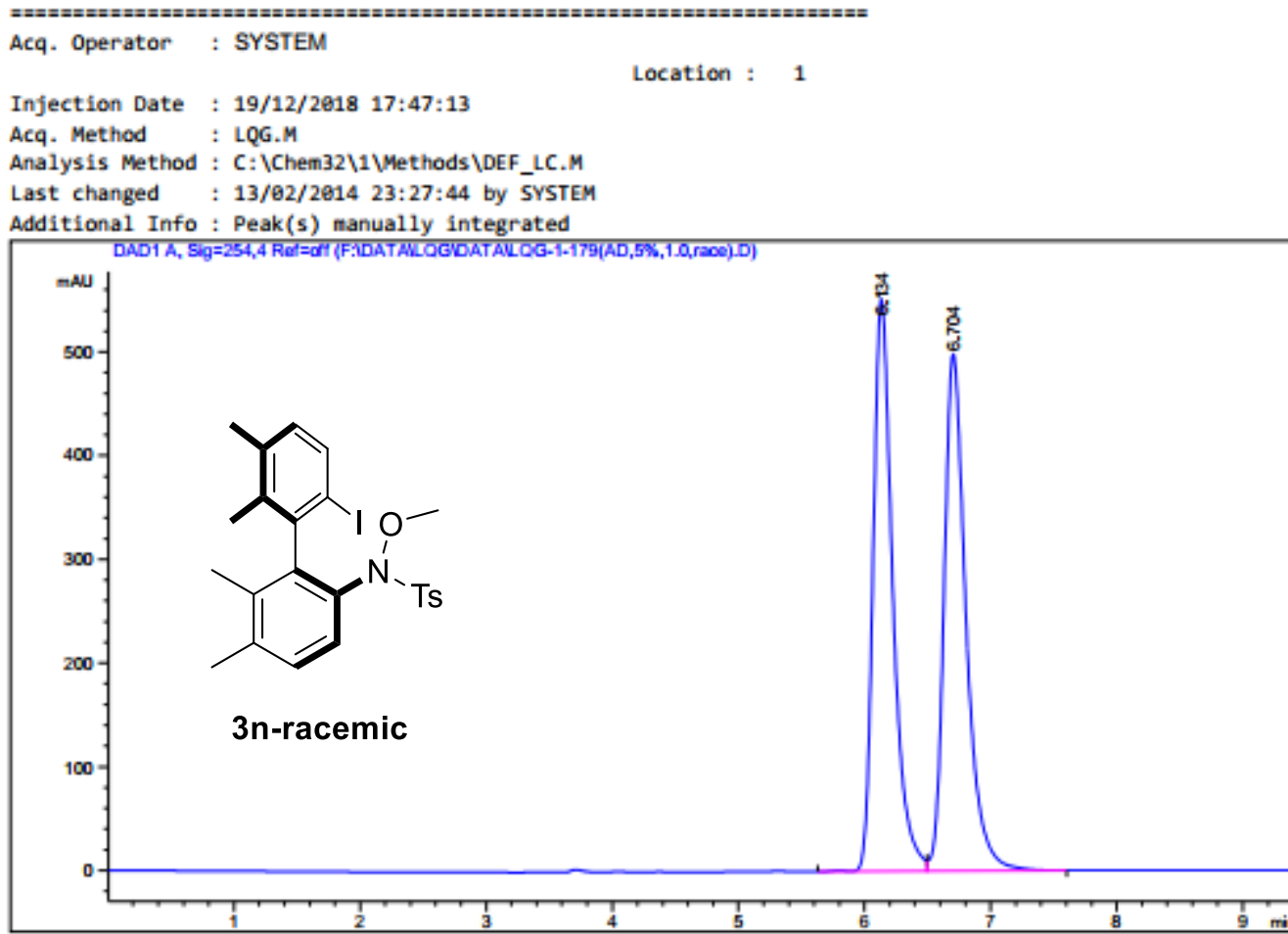

Area Percent Report

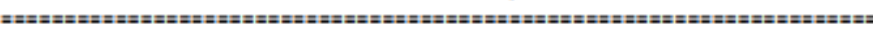

$\begin{array}{lll}\text { Sorted By } & : & \text { Signal } \\ \text { Multiplier } & : & 1.0980 \\ \text { Dilution } & : & 1 . e 980\end{array}$

Use Multiplier \& Dilution Factor with ISTDS

Signal 1: DAD1 A, Sig $=254,4$ Ref $=0$ ff

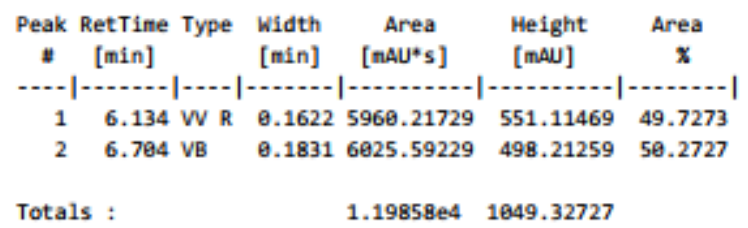




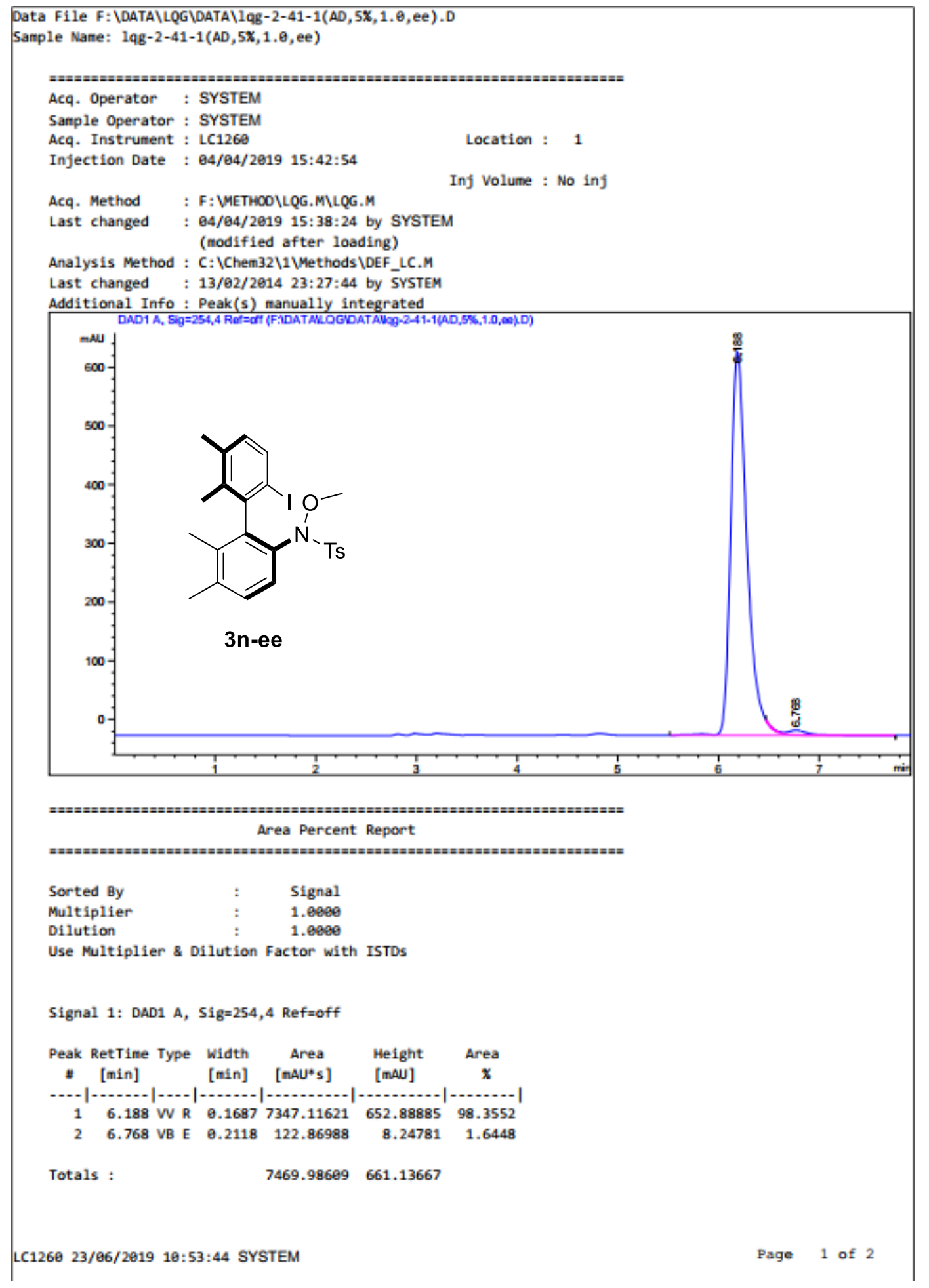


<Sample Information>

Sample Name : lqg-2-153-1(AD,5\%,1.0,rac)

Sample ID

Data Filename : Iqg-2-153-1(AD, $5 \%, 1.0$, rac).lcd

Method Filename : GWJ single.Icm

Batch Filename

Vial \#

$1-1$

Injection Volume $\quad: 15 \mathrm{uL}$

Date Acquired : 5/17/2019 10:43:47 AM

Date Processed : : 5/17/2019 10:58:50 AM

Sample Type : Unknown

Acquired by : : System Administrator

Processed by : : System Administrator

\section{$<$ Chromatogram>}

$\mathrm{mV}$

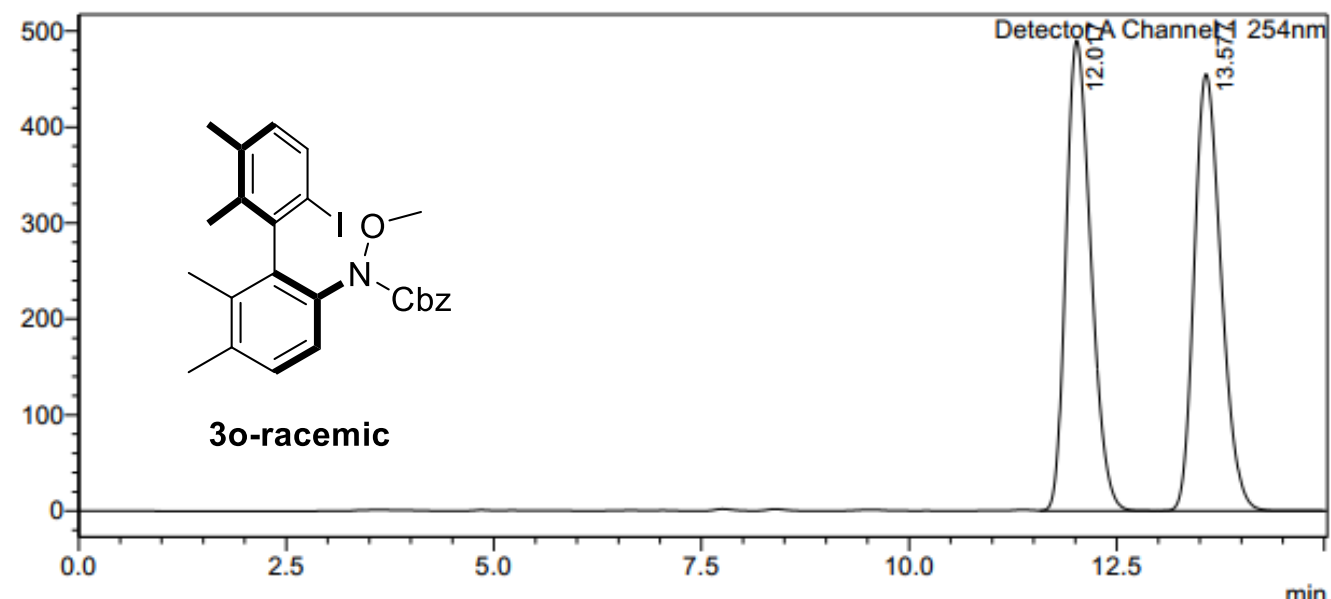

<Peak Table>

Detector A Channel $1254 \mathrm{~nm}$

\begin{tabular}{|r|r|r|r|r|r|r|r|}
\hline Peak\# & Ret. Time & \multicolumn{1}{c|}{ Area } & Height & Conc. & Unit & Mark & Name \\
\hline 1 & 12.017 & 10246056 & 489727 & 49.902 & & & \\
\hline 2 & 13.577 & 10286221 & 454864 & 50.098 & & $\mathrm{~V}$ & \\
\hline Total & & 20532276 & 944590 & & & & \\
\hline
\end{tabular}


<Sample Information>

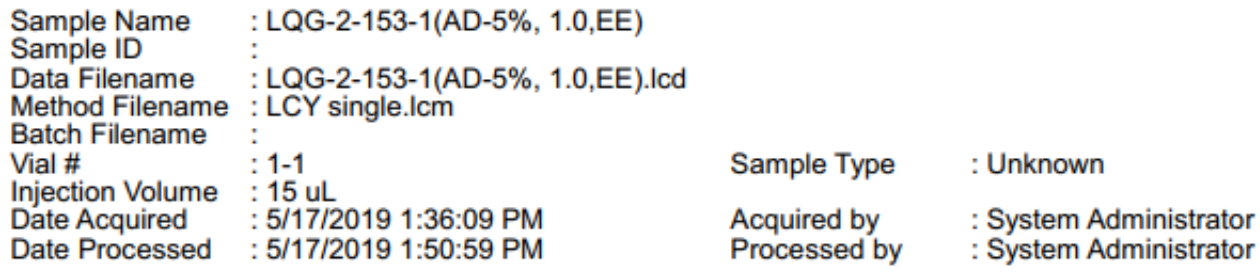

Sample Type : : Unknown

Acquired by : : System Administrator Processed by : : System Administrator

\section{$<$ Chromatogram $>$}

$\mathrm{mV}$

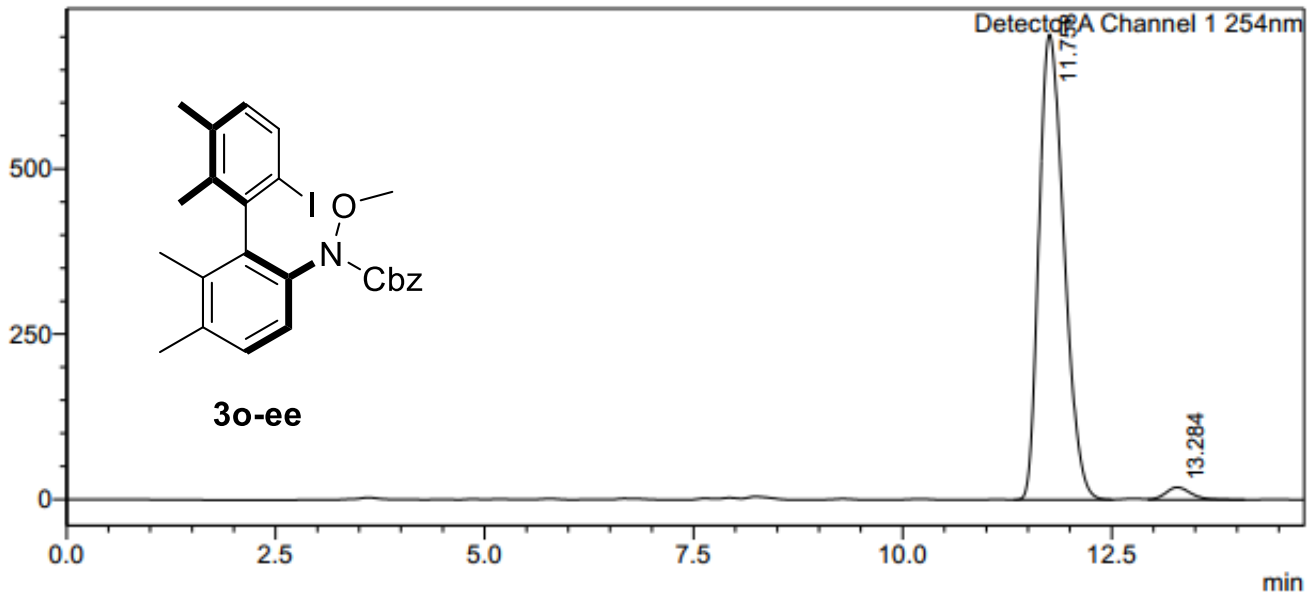

<Peak Table>

Detector A Channel $1254 \mathrm{~nm}$

\begin{tabular}{|r|r|r|r|r|r|r|r|}
\hline Peak\# & Ret. Time & \multicolumn{1}{|c|}{ Area } & \multicolumn{1}{c|}{ Height } & \multicolumn{1}{c|}{ Conc. } & Unit & Mark & Name \\
\hline 1 & 11.758 & 14715431 & 704132 & 97.330 & & & \\
\hline 2 & 13.284 & 403736 & 18833 & 2.670 & & & \\
\hline Total & & 15119168 & 722966 & & & & \\
\hline
\end{tabular}


Data File F: \DATAYLQG DATAYLQG-2-41-2(AD, 5X,1.6, race).D

Sample Name: LQG-2-41-2(AD, 5X,1. $\theta$, race)
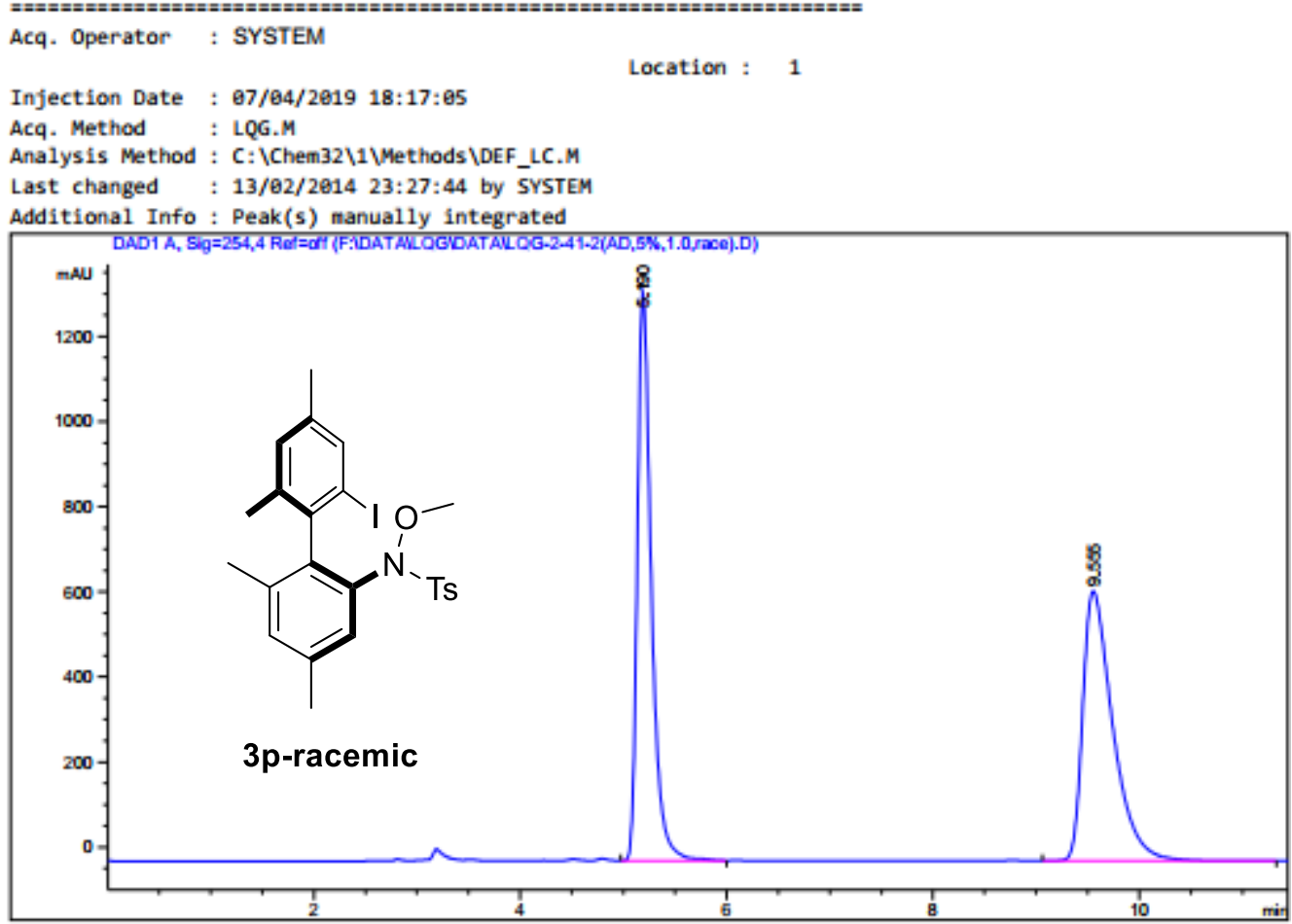

Area Percent Report

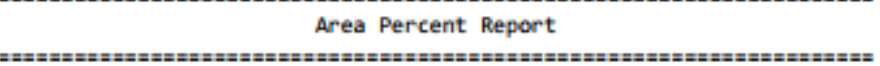

$\begin{array}{lll}\text { Sorted By } & : & \text { Signal } \\ \text { Multiplier } & : & 1.0980 \\ \text { Dilution } & : & 1.6980\end{array}$

Use Multiplier \& Dilution Factor with ISTDs

Signal 1: DAD1 A, Sig $=254,4$ Ref $=$ off

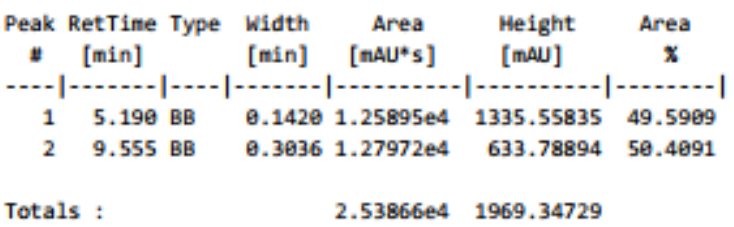


Data File F: \DATAYLQG DATA\lqg-2-41-2-2(AD, 5X,1.0, ee).D

Sample Name: 1qg-2-41-2-2(AD,5\%,1. $\theta$,ee)
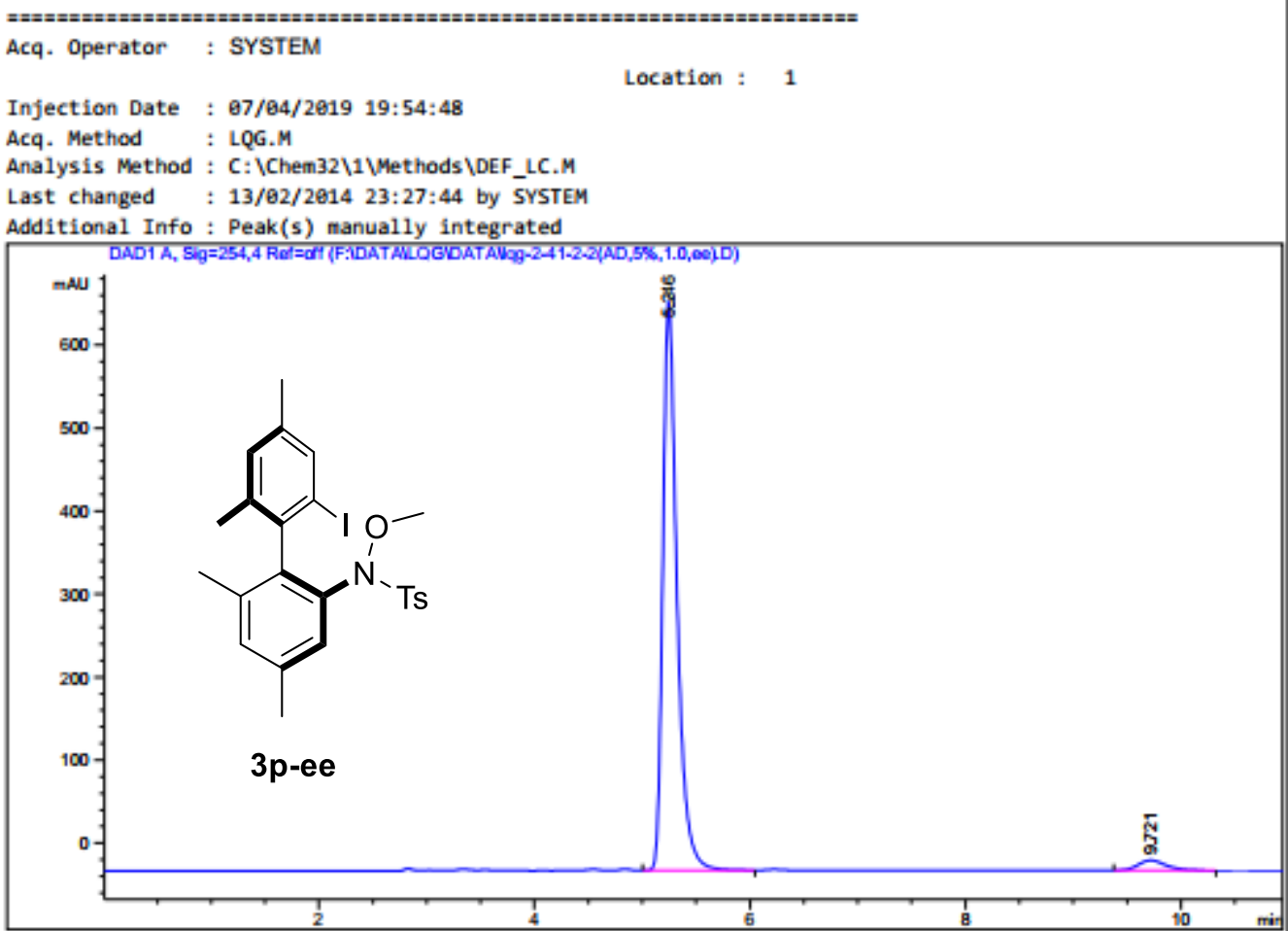

Area Percent Report

\begin{tabular}{|c|c|c|}
\hline Sorted By & : & Signal \\
\hline Multiplier & : & 1.6909 \\
\hline Dilution & : & $1 . e 900$ \\
\hline
\end{tabular}

Use Multiplier \& Dilution Factor with ISTDs

Signal 1: DAD1 A, Sig $=254,4$ Ref $=0$ ff

\begin{tabular}{|c|c|c|c|c|c|}
\hline $\begin{array}{c}\text { Peak } \\
y\end{array}$ & $\begin{array}{l}\text { RetTime Type } \\
\text { [min] }\end{array}$ & $\begin{array}{l}\text { Width } \\
\text { [min] }\end{array}$ & $\begin{array}{c}\text { Area } \\
\text { [nAU*s] }\end{array}$ & $\begin{array}{l}\text { Height } \\
\text { [mall] }\end{array}$ & $\begin{array}{c}\text { Area } \\
\mathbf{x}\end{array}$ \\
\hline & 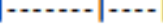 & (1) & | & 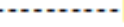 & 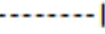 \\
\hline 1 & $5.246 \mathrm{BB}$ & 0.1370 & 6274.51465 & 684.17462 & 96.7616 \\
\hline 2 & $9.721 \mathrm{BB}$ & 0.2693 & 209.99706 & 11.93598 & 3.2384 \\
\hline Tota & & & 6484.51170 & 696.11061 & \\
\hline
\end{tabular}

$* * *$ End of Report *** 


\section{<Sample Information>}

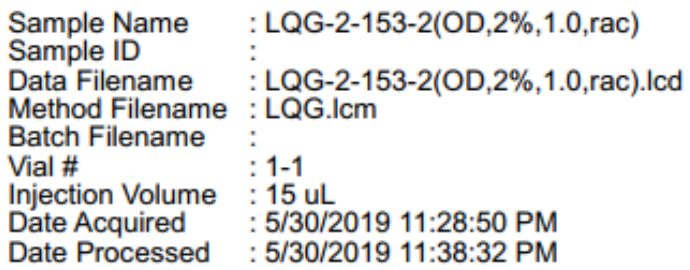

\section{$<$ Chromatogram>}

$\mathrm{mV}$

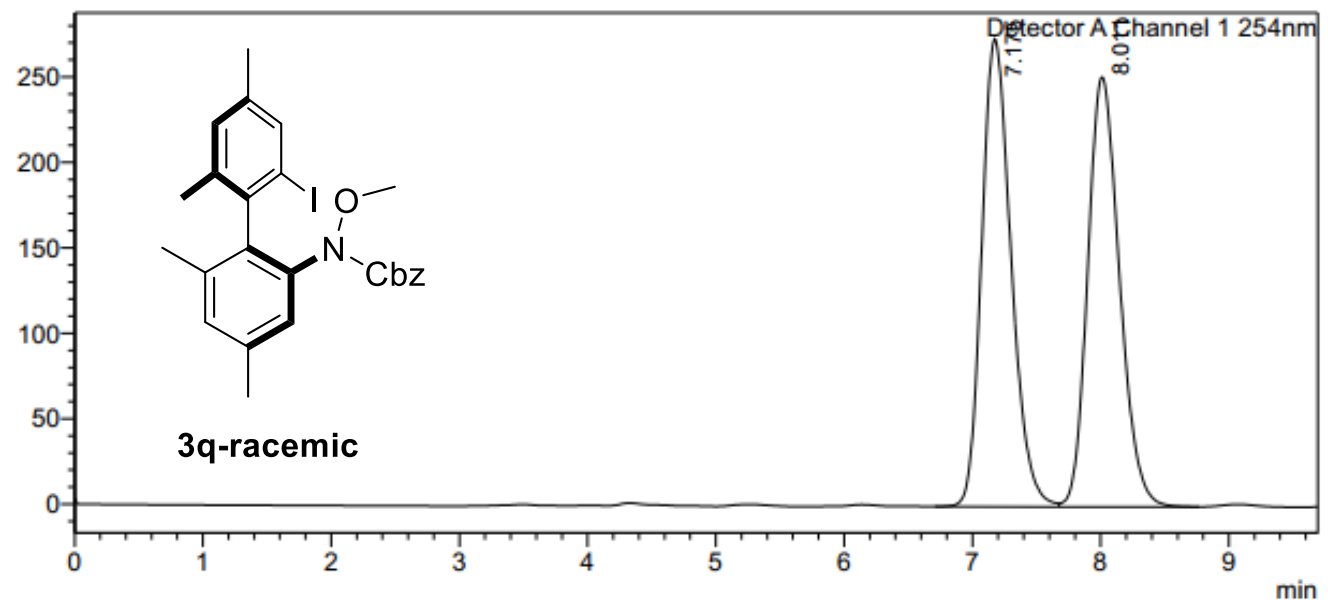

\section{<Peak Table>}

Detector A Channel $1254 \mathrm{~nm}$

\begin{tabular}{|r|r|r|r|r|r|r|r|}
\hline Peak\# & Ret. Time & Area & \multicolumn{1}{c|}{ Height } & \multicolumn{1}{c|}{ Conc. } & Unit & Mark & Name \\
\hline 1 & 7.175 & 4366517 & 273718 & 50.225 & & & \\
\hline 2 & 8.011 & 4327365 & 251313 & 49.775 & & V & \\
\hline Total & & 8693881 & 525031 & & & & \\
\hline
\end{tabular}


<Sample Information>

Sample Name : LQG-2-153-2(OD,2\%,1.0,EE)

Sample ID

Data Filename : LQG-2-153-2(OD,2\%,1.0,EE).Icd

Method Filename : LQG.Icm

Batch Filename

Vial \#

$: 1-1$

Injection Volume : 15 uL

Date Acquired : 5/30/2019 11:43:45 PM

Date Processed : :5/30/2019 11:52:54 PM

Sample Type : : Unknown

Acquired by : : System Administrator Processed by : System Administrator

\section{$<$ Chromatogram>}

$\mathrm{mV}$

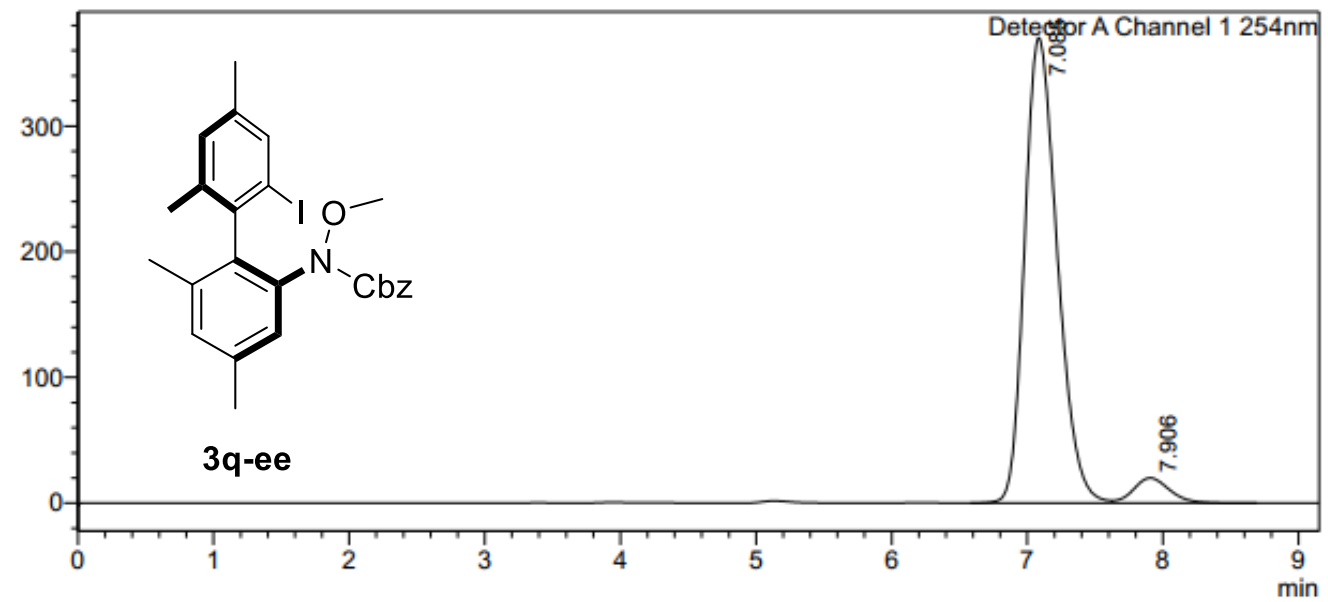

<Peak Table>

Detector A Channel $1254 \mathrm{~nm}$

\begin{tabular}{|r|r|r|r|r|r|r|r|}
\hline Peak\# & Ret. Time & \multicolumn{1}{|c|}{ Area } & \multicolumn{1}{c|}{ Height } & \multicolumn{1}{c|}{ Conc. } & Unit & Mark & Name \\
\hline 1 & 7.085 & 6222239 & 370115 & 94.360 & & & \\
\hline 2 & 7.906 & 371895 & 19955 & 5.640 & & $\mathrm{~V}$ & \\
\hline Total & & 6594133 & 390070 & & & & \\
\hline
\end{tabular}




\section{<Sample Information>}

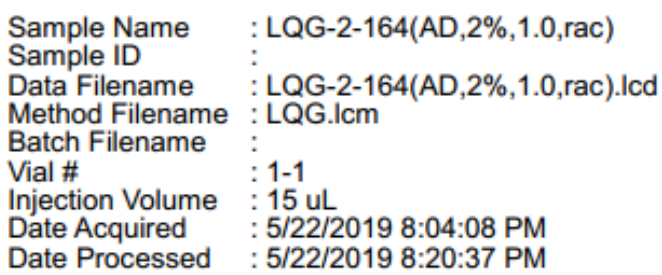

Acquired by : : System Administrator Processed by : :System Administrator

\section{$<$ Chromatogram $>$}

$\mathrm{mV}$

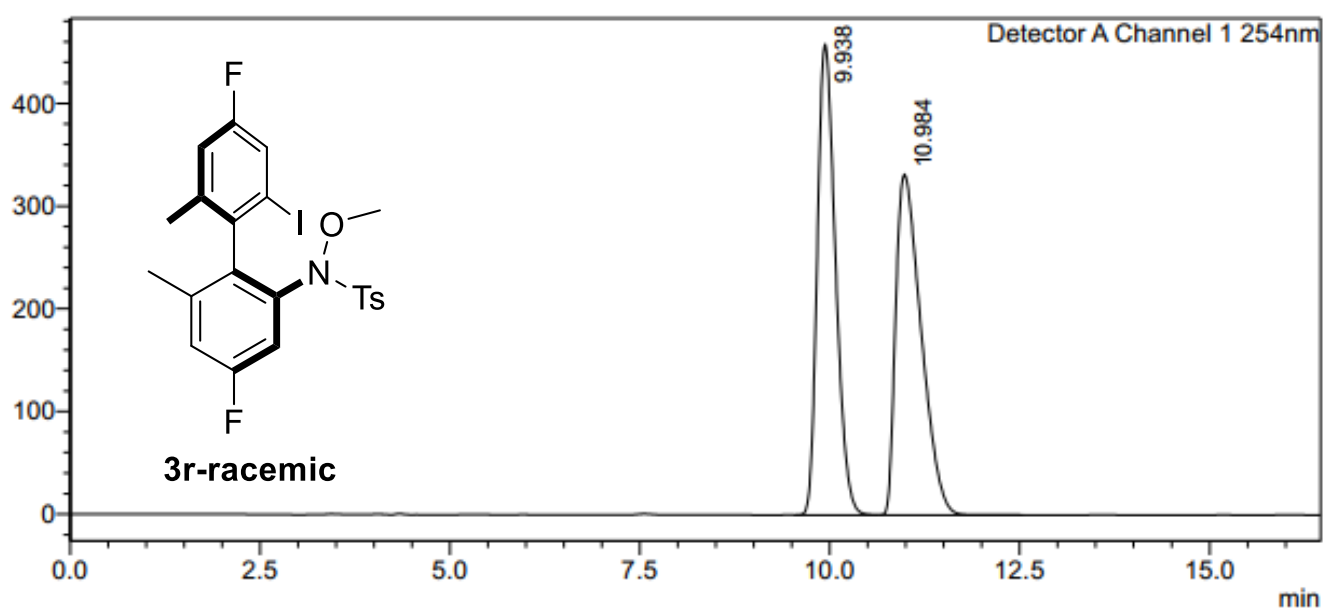

<Peak Table>

Detector A Channel $1254 \mathrm{~nm}$

\begin{tabular}{|r|r|r|r|r|r|r|r|}
\hline Peak\# & Ret. Time & \multicolumn{1}{c|}{ Area } & Height & Conc. & Unit & Mark & Name \\
\hline 1 & 9.938 & 7647447 & 458004 & 49.819 & & V & \\
\hline 2 & 10.984 & 7703083 & 331421 & 50.181 & & SV & \\
\hline Total & & 15350530 & 789425 & & & & \\
\hline
\end{tabular}


<Sample Information>

Sample Name : LQG-2-164(AD,2\%,1.0,EE)

Sample ID

Data Filename : LQG-2-164(AD,2\%,1.0,EE).Icd

Method Filename : LQG.Icm

Batch Filename :

Vial \# : :1-1

Injection Volume : $15 \mathrm{uL}$

Date Acquired : 5/22/2019 8:25:34 PM

Sample Type : Unknown

Date Processed $\quad$ : 5/22/2019 8:38:53 PM

Acquired by : : System Administrator Processed by : System Administrator

\section{<Chromatogram>}

$\mathrm{mV}$

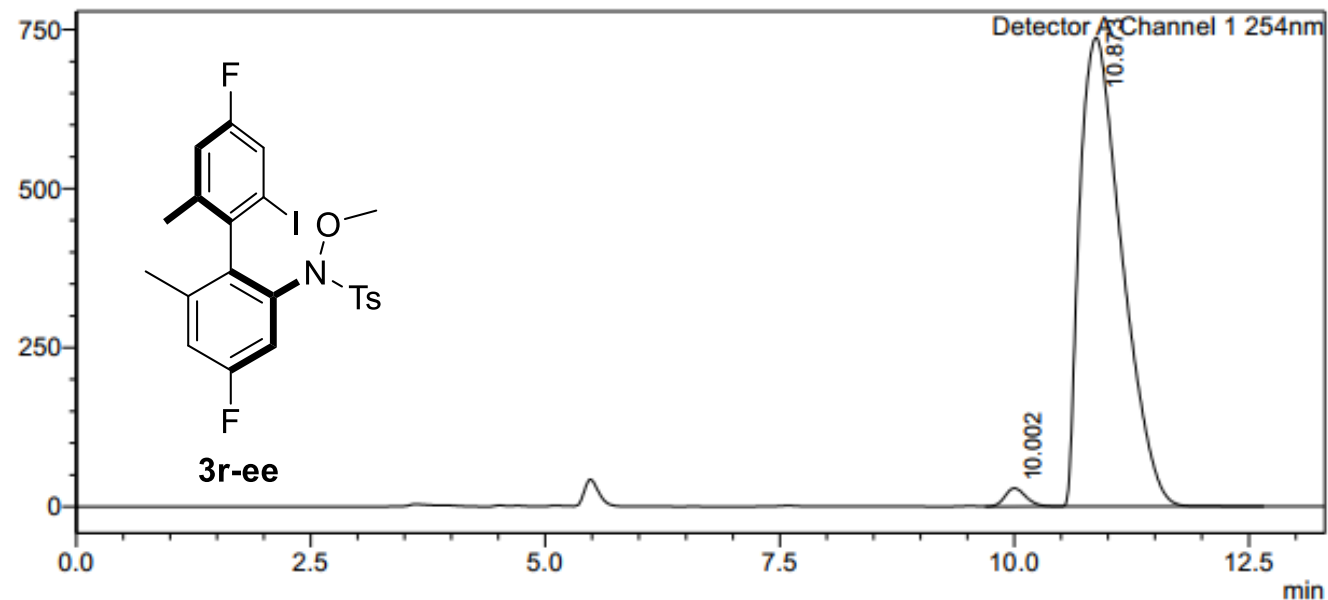

<Peak Table>

Detector A Channel $1254 \mathrm{~nm}$

\begin{tabular}{|r|r|r|r|r|r|r|r|}
\hline Peak\# & Ret. Time & \multicolumn{1}{|c|}{ Area } & \multicolumn{1}{c|}{ Height } & \multicolumn{1}{c|}{ Conc. } & Unit & Mark & Name \\
\hline 1 & 10.002 & 454248 & 28782 & 1.936 & & & \\
\hline 2 & 10.873 & 23012579 & 737071 & 98.064 & & V & \\
\hline Total & & 23466827 & 765852 & & & & \\
\hline
\end{tabular}




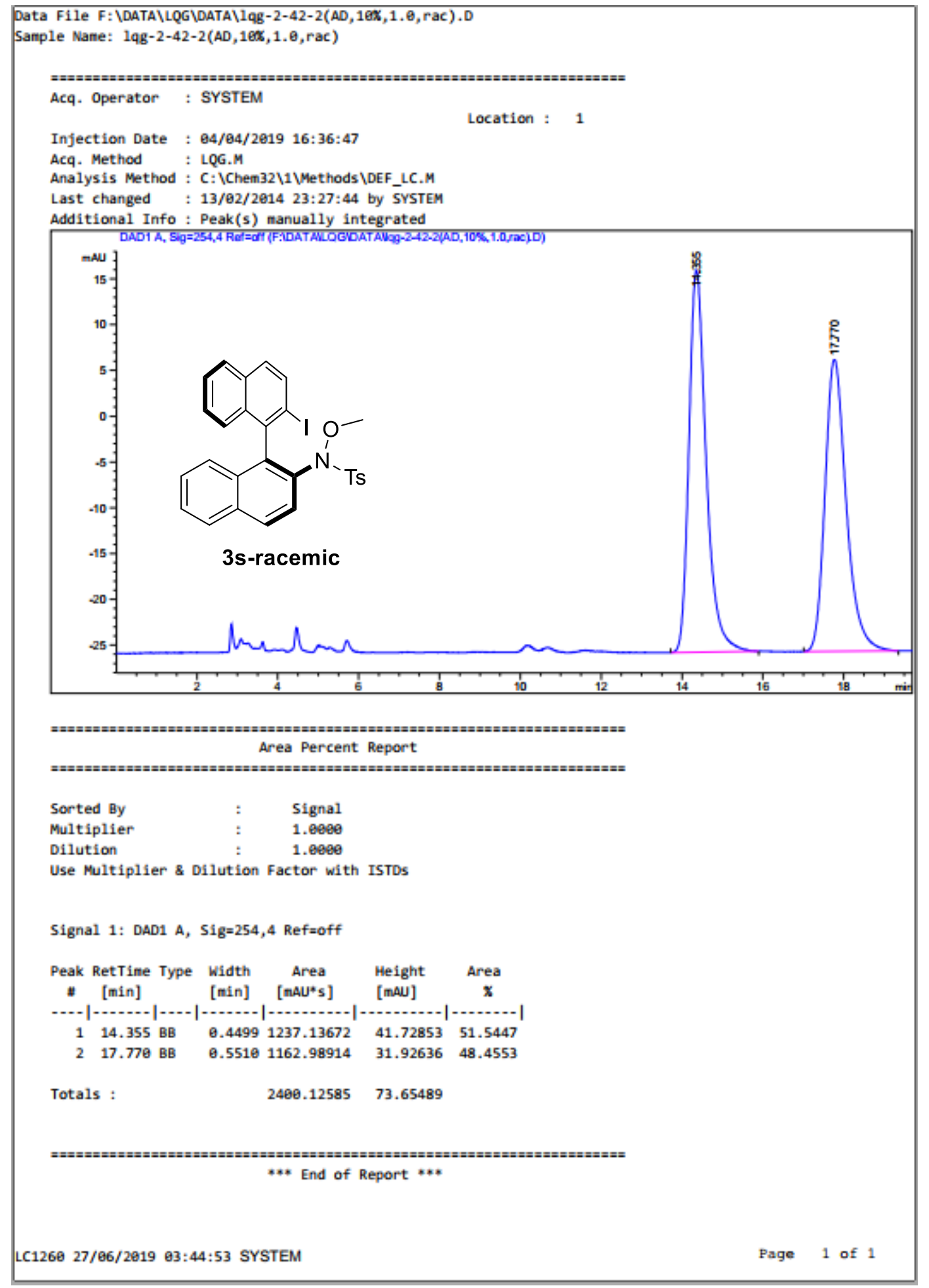




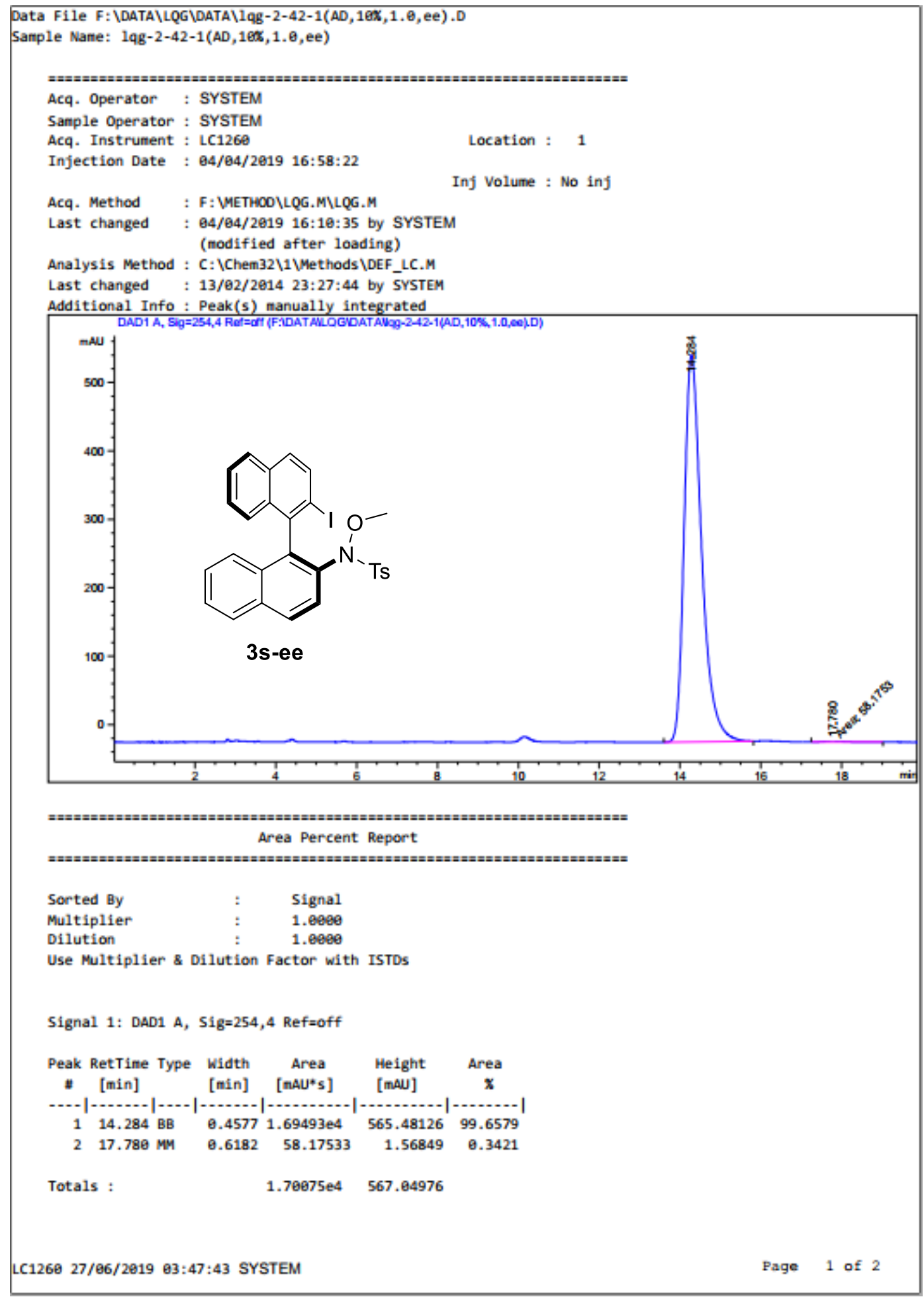


Data File F: \DATA\LQG DATA\LQG-2-49(AD, 5X,1. 6 , raec).D

Sample Name: $L Q G-2-49(A D, 5 \times, 1.6, \mathrm{raeC})$

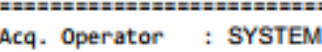

Sample Operator : SYSTEM

Acq. Instrument : LC1260

Injection Date : $97 / 64 / 2819$ 18:44:01

Location : 1

Acq. Method : F: METHOOYLQG.MILQG.M

Inj Volume : No inj

Last changed : 07/04/2019 18:14:18 by SYSTEM

(modified after loading)

Analysis Method : C: \Chem32\1 Methods \DEF_LC.M

Last changed : 13/02/2814 $23: 27: 44$ by SYSTEM

Additional Info: Peak(s) manually integrated

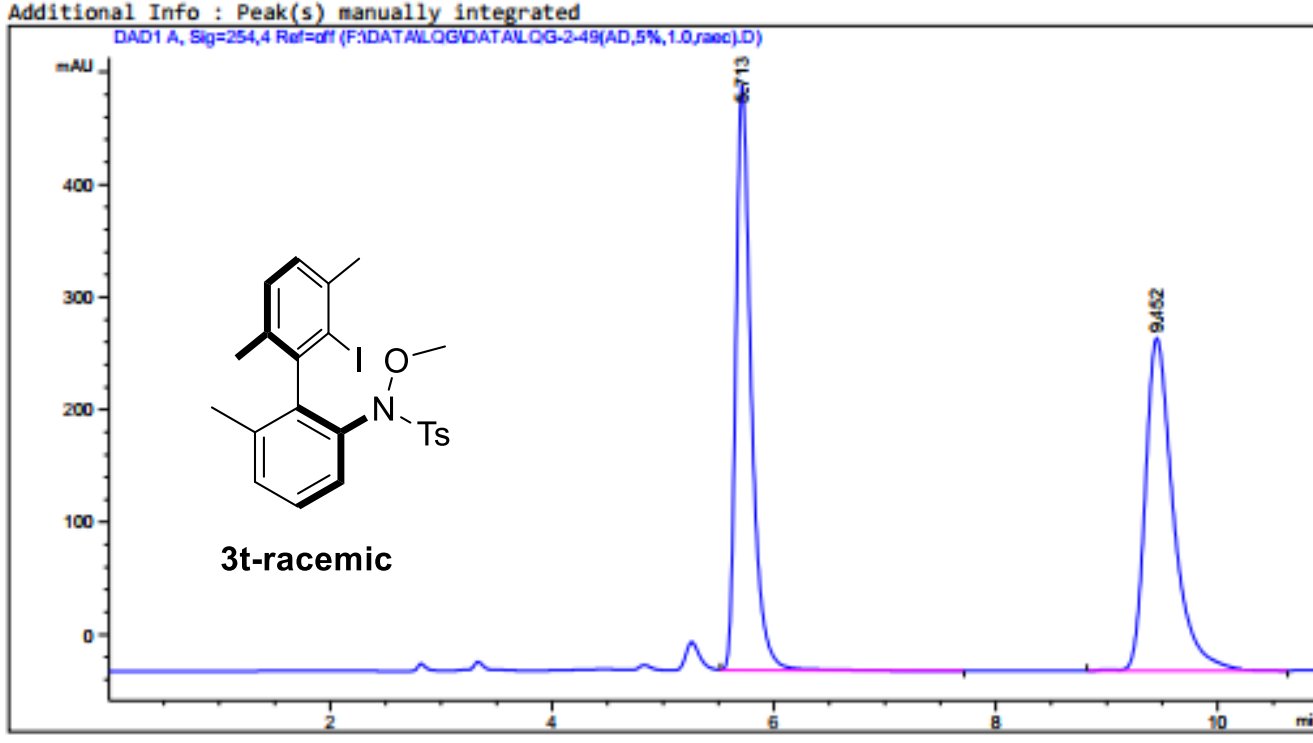

Area Percent Report

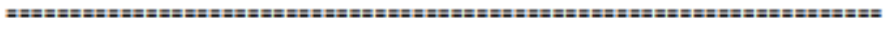

Sorted By : : Signal

Multiplier $\quad: \quad 1.6090$

Dilution

Use Multiplier \& Dilution Factor with ISTDs

Signal 1: DAD1 A, Sig $=254,4$ Ref $=0$ ff

\begin{tabular}{|c|c|c|c|c|c|}
\hline $\begin{array}{c}\text { Peak } \\
y\end{array}$ & $\begin{array}{l}\text { RetTime Type } \\
\text { [min] }\end{array}$ & $\begin{array}{l}\text { Width } \\
\text { [min] }\end{array}$ & $\begin{array}{c}\text { Area } \\
\text { [nAU*s] }\end{array}$ & $\begin{array}{l}\text { Height } \\
\text { [mall] }\end{array}$ & $\begin{array}{c}\text { Area } \\
\mathbf{x}\end{array}$ \\
\hline 1 & 713 VB & 56 & 5062.84375 & 519.84351 & 49.3886 \\
\hline & & & & 295,46429 & \\
\hline
\end{tabular}

Totals :

$1.02510 \mathrm{e} 4 \quad 815.30780$ 


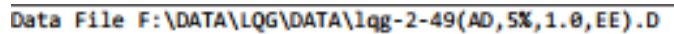

Sample Name: $1 q g-2-49(A D, 5 X, 1.6, E E)$
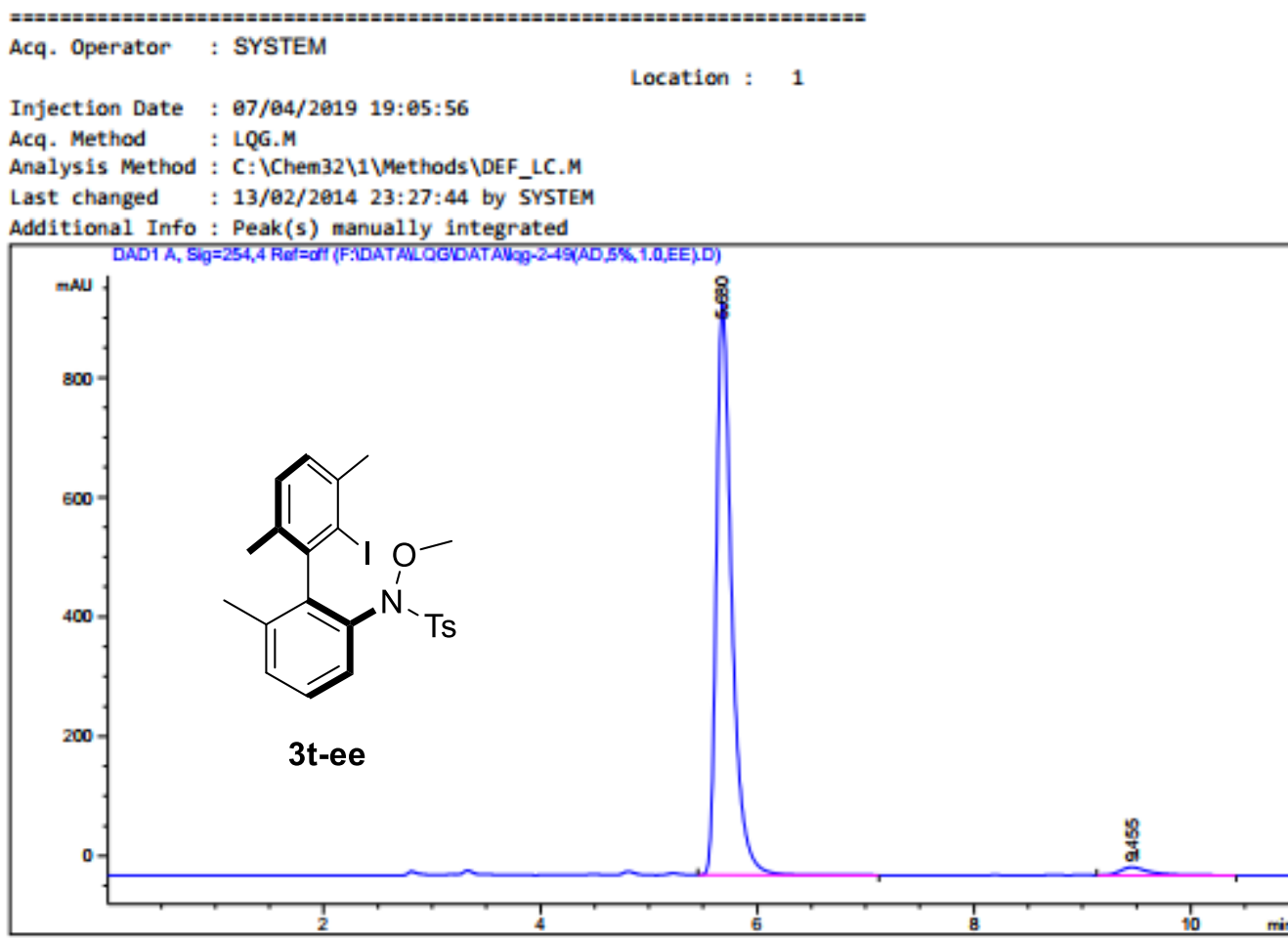

Area Percent Report

Location : 1

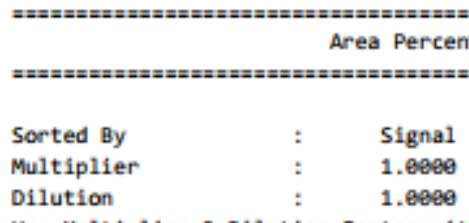

Use Multiplier \& Dilution Factor with ISTDs

Signal 1: DAD1 A, Sig $=254,4$ Ref $=0$ ff

\begin{tabular}{cccccc}
$\begin{array}{c}\text { Peak RetTime Type } \\
\text { [min] }\end{array}$ & $\begin{array}{c}\text { Width } \\
\text { [min] }\end{array}$ & $\begin{array}{c}\text { Area } \\
\text { [mAU*s] }\end{array}$ & $\begin{array}{l}\text { Height } \\
\text { [maU] }\end{array}$ & $\begin{array}{c}\text { Area } \\
\mathbf{x}\end{array}$ \\
\hline 1 & 5.680 VB & 0.1497 & 9482.16504 & 955.73468 & 97.4887 \\
2 & 9.455 BB & 0.2796 & 244.25656 & 13.27935 & 2.5113 \\
& & & & & \\
Totals : & & 9726.42160 & 969.01403 &
\end{tabular}

$* * *$ End of Report *** 
<Sample Information>

Sample Name

Sample ID

: LQG-2-140(ID,5\%,1.0,RAC)

Data Filen

: LQG-2-140(ID,5\%,1.0,RAC).Icd

Method Filename : LQG.Icm

Batch Filename

Vial \#

$: 1-1$

Injection Volume : 15 uL

Date Acquired $\quad:$ 5/30/2019 7:45:39 PM

Date Processed $\quad$ : 5/30/2019 8:13:02 PM

Sample Type : : Unknown

Acquired by : : System Administrator

Processed by : :System Administrator

\section{$<$ Chromatogram>}

$\mathrm{mV}$

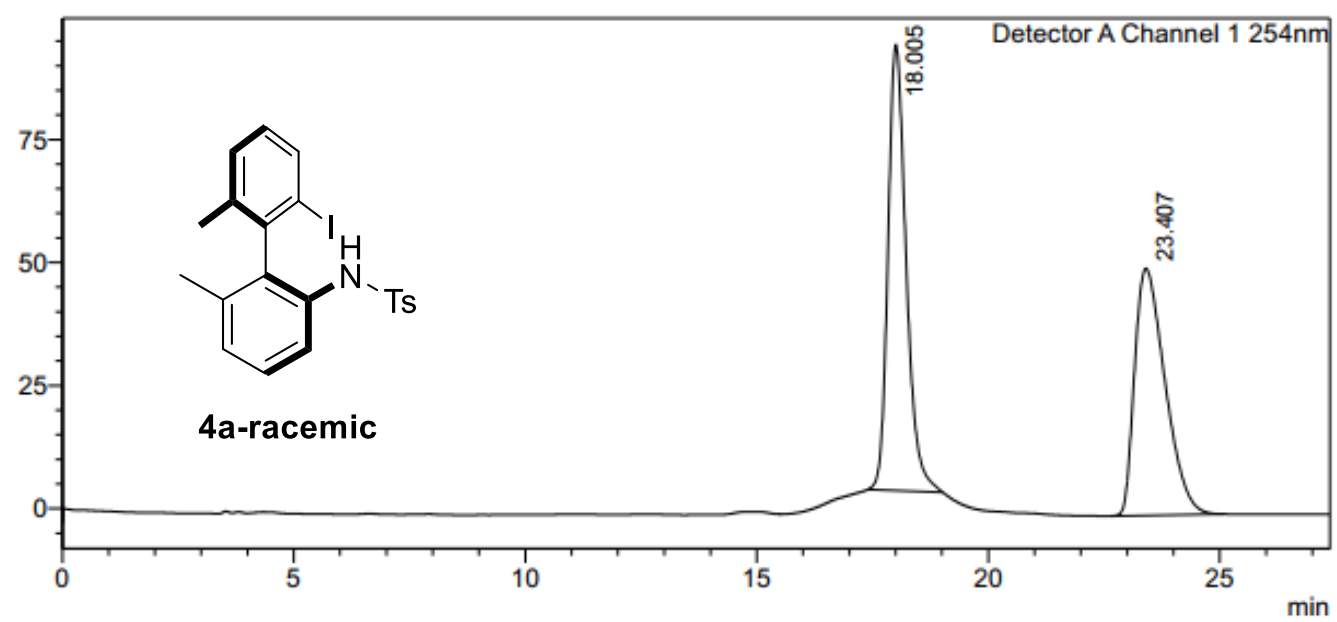

<Peak Table>

Detector A Channel $1254 \mathrm{~nm}$

\begin{tabular}{|r|r|r|r|r|r|r|r|}
\hline Peak\# & Ret. Time & Area & Height & Conc. & Unit & Mark & Name \\
\hline 1 & 18.005 & 2477397 & 90555 & 51.904 & & $\mathrm{M}$ & \\
\hline 2 & 23.407 & 2295613 & 50168 & 48.096 & & & \\
\hline Total & & 4773009 & 140723 & & & & \\
\hline
\end{tabular}


<Sample Information>

Sample Name : LQG-2-183(ID,5\%,1.0,ee)

Sample ID

Data Filename : LQG-2-183(ID,5\%,1.0,ee).Icd

Method Filename : LQG.Icm

Batch Filename

Vial \# $\quad: 1-1$

Injection Volume : $15 \mathrm{uL}$

Date Acquired : 5/30/2019 8:19:43 PM

Date Processed $\quad: 5 / 30 / 2019$ 8:46:10 PM

Sample Type : Unknown

Acquired by : System Administrator Processed by :System Administrator

\section{$<$ Chromatogram>}

$\mathrm{mV}$

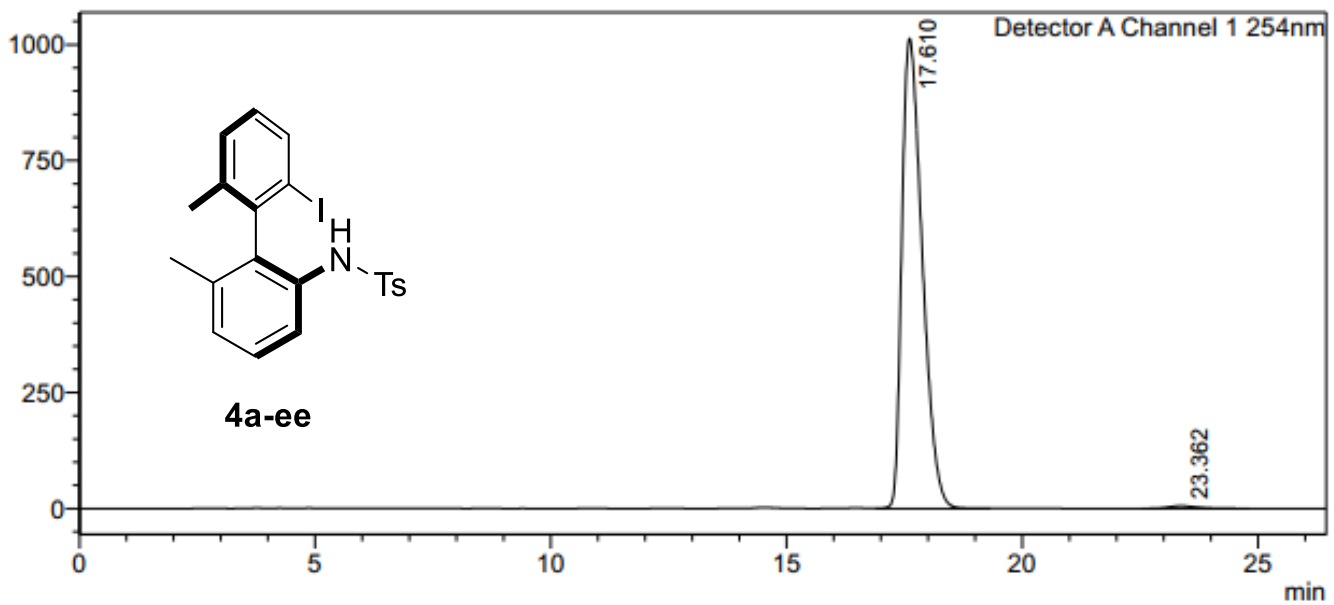

<Peak Table>

Detector A Channel $1254 \mathrm{~nm}$

\begin{tabular}{|r|r|r|r|r|r|r|r|}
\hline Peak\# & Ret. Time & \multicolumn{1}{|c|}{ Area } & \multicolumn{1}{c|}{ Height } & \multicolumn{1}{c|}{ Conc. } & Unit & Mark & Name \\
\hline 1 & 17.610 & 30483390 & 1012893 & 99.267 & & & \\
\hline 2 & 23.362 & 225103 & 5555 & 0.733 & & S & \\
\hline Total & & 30708493 & 1018448 & & & & \\
\hline
\end{tabular}


Data File F: \DATA\LQG DATA $1 \mathrm{qg}-3-21(00,99 \mathrm{x}, 1.6, \mathrm{rac})-2 . \mathrm{D}$

Sample Name: $1 \mathrm{qg}-3-21(00,99 \mathrm{x}, 1.6, \mathrm{rac})-2$
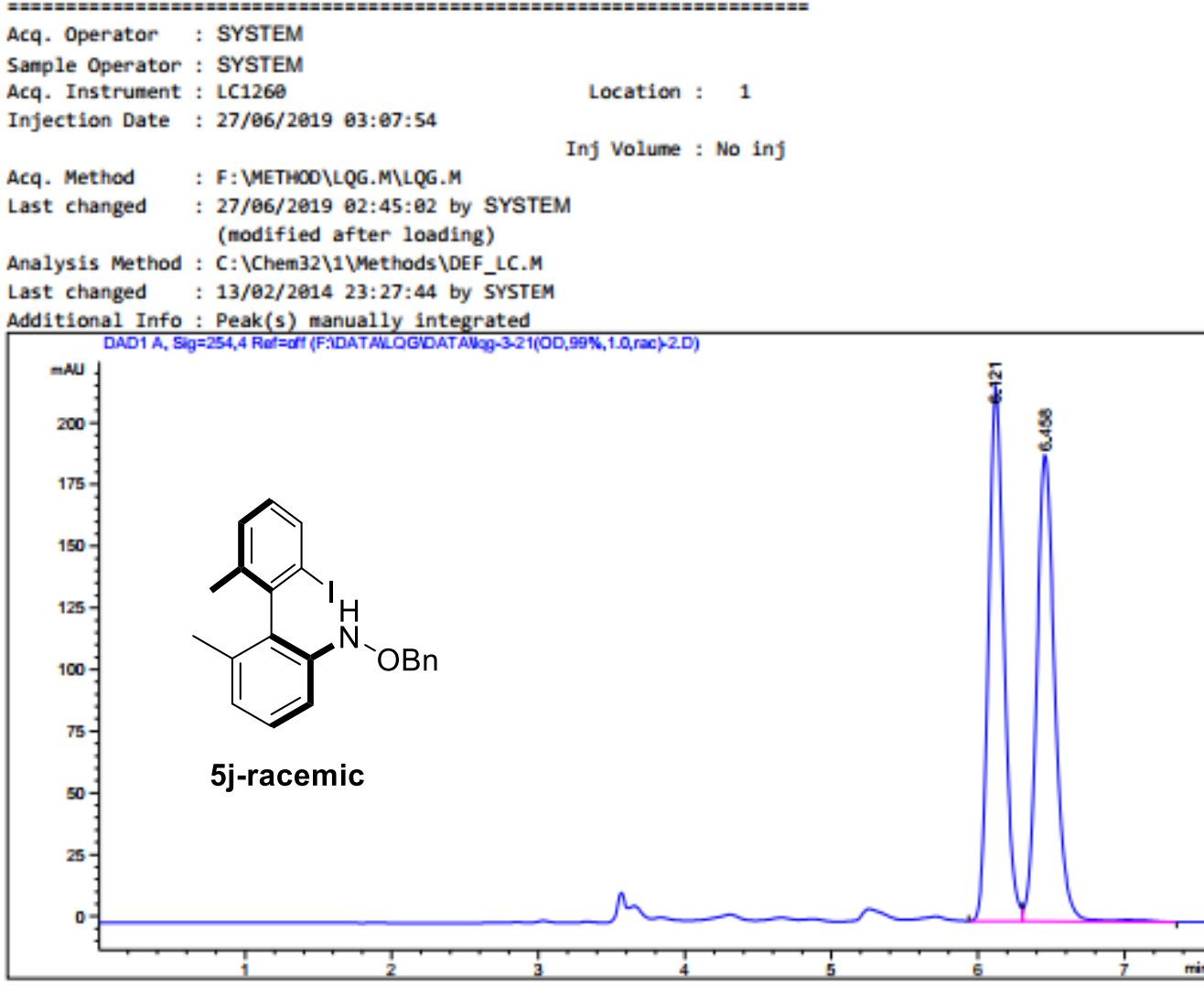

Area Percent Report

\begin{tabular}{|c|c|c|}
\hline Sorted By & : & Signal \\
\hline Multiplier & : & 1.0990 \\
\hline Dilution & : & 1.0980 \\
\hline
\end{tabular}

Use Multiplier \& Dilution Factor with ISTDs

Signal 1: DAD1 A, Sig $=254,4$ Ref $=0$ ff

\begin{tabular}{cccccc}
$\begin{array}{c}\text { Peak RetTime Type width } \\
\text { [min] }\end{array}$ & $\begin{array}{c}\text { Area } \\
\text { [min] }\end{array}$ [mAU*s] & $\begin{array}{c}\text { Height } \\
\text { [maU] }\end{array}$ & $\begin{array}{c}\text { Area } \\
\mathbf{x}\end{array}$ \\
\hline 1 & 6.121 BV & 0.1135 & 1583.65442 & 216.01329 & 49.2245 \\
2 & 6.458 WV R & 0.1312 & 1633.55298 & 189.10907 & 50.7755
\end{tabular}

Totals :

$3217.28740 \quad 495.12236$ 
Data File F: \DATAYLQG DATA $1 \mathrm{qg}-3-22(00,99 \mathrm{x}, 1 . \theta, \mathrm{EE})-2 . \mathrm{D}$

Sample Name: $1 \mathrm{qg}-3-22(00,99 \mathrm{x}, 1.6, \mathrm{EE})-2$
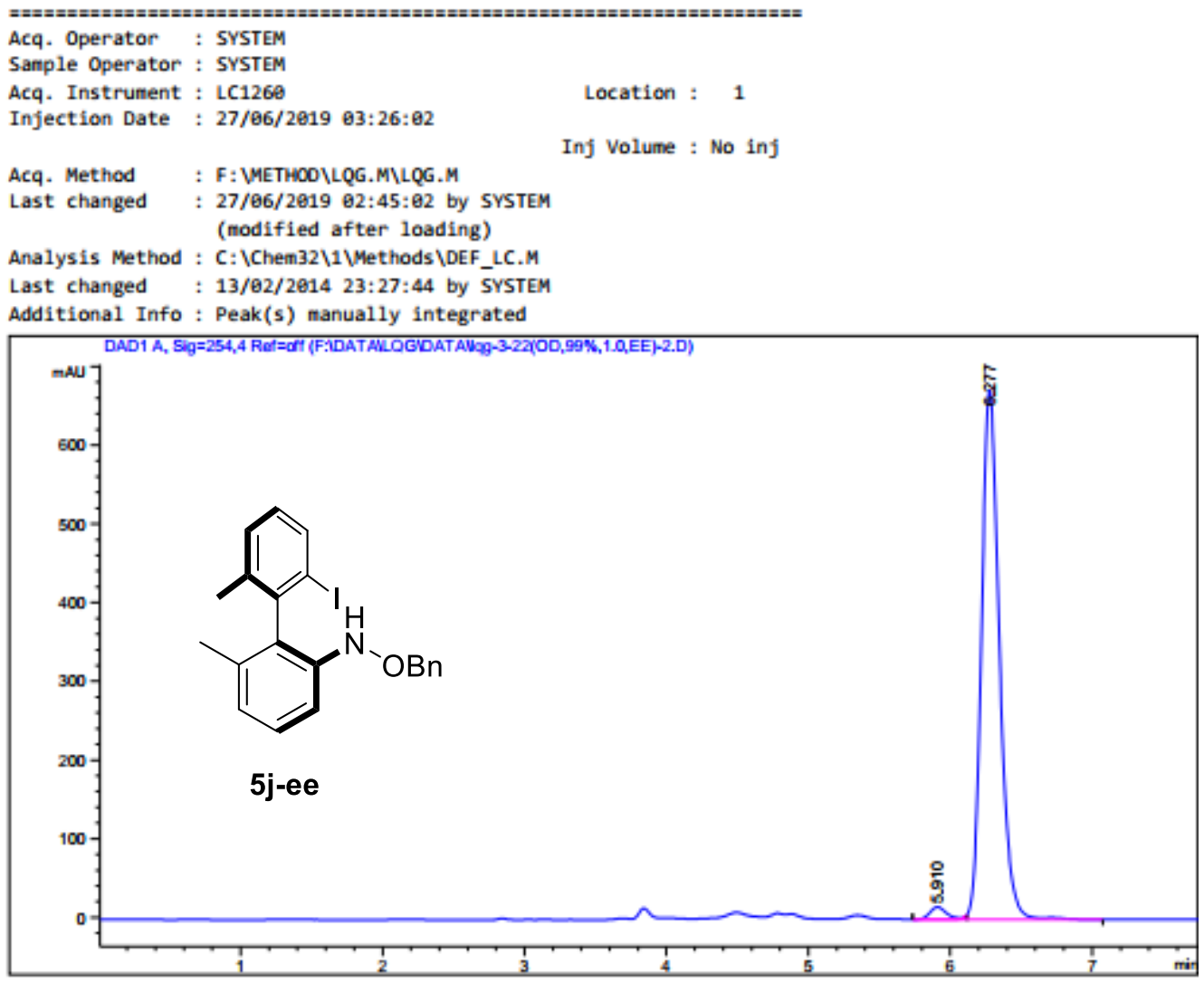

Area Percent Report

\begin{tabular}{|c|c|c|}
\hline Sorted By & : & Sign \\
\hline ultiplier & : & \\
\hline & : & \\
\hline
\end{tabular}

Use Multiplier \& Dilution Factor with ISTDS

Signal 1: DAD1 A, Sig $=254,4$ Ref $=$ off

\begin{tabular}{cccccc}
$\begin{array}{c}\text { Peak RetTime Type Width } \\
\text { [min] }\end{array}$ [min] & $\begin{array}{c}\text { Area } \\
\text { [mAU*s] }\end{array}$ & $\begin{array}{c}\text { Height } \\
\text { [mAU] }\end{array}$ & \multicolumn{1}{c}{$\begin{array}{c}\text { Area } \\
\mathbf{X}\end{array}$} \\
\hline 1 & 5.910 BV E & 0.1231 & 127.86519 & 15.68876 & 2.1217 \\
\hline 2 & 6.277 WV R & 0.1338 & 5898.67578 & 672.73169 & 97.8783
\end{tabular}

Totals :

$6026.54997 \quad 688.42645$ 
Data File F: \DATAYLQG DATAYlag-3-18-1(AD, 1X,1.6, RAC).D

Sample Name: 1qg-3-18-1(AD, 1X,1.0, RAC)

Acq. Operator : SYSTEM

Sample Operator : SYSTEM

Acq. Instrument : LC1260

Injection Date : 27/06/2819 04:12:45

Location : 1

Inj Volume : No inj

Acq. Method : F: METHOOYLQG.MILQG.M

Last changed : 27/06/2019 $04: 02: 06$ by SYSTEM

(modified after loading)

Analysis Method : C: \Chem32\1\Methods \DEF LC.M

Last changed : 13/02/2014 23:27:44 by SYSTEM

Additional Info : Peak(s) manually integrated

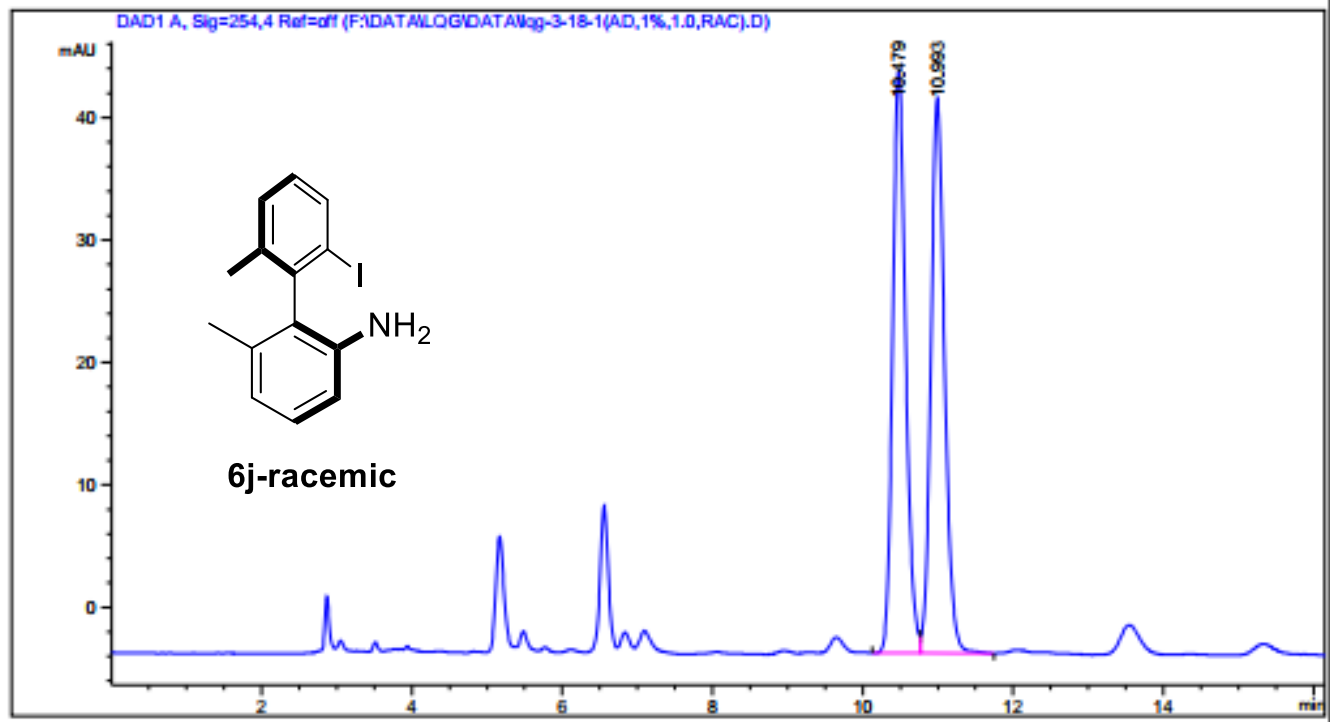

Area Percent Report

Sorted By : Signal

Multiplier : $\quad 1.6900$

Dilution : 1.6990

Use Multiplier \& Dilution Factor with ISTDs

Signal 1: DAD1 A, Sig $=254,4$ Ref $=$ off

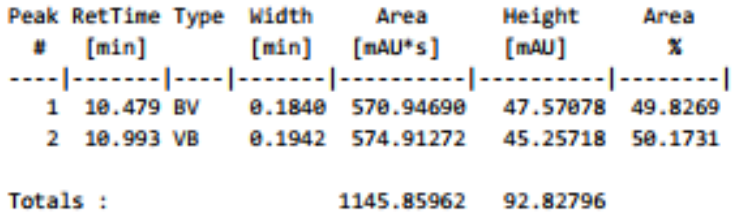




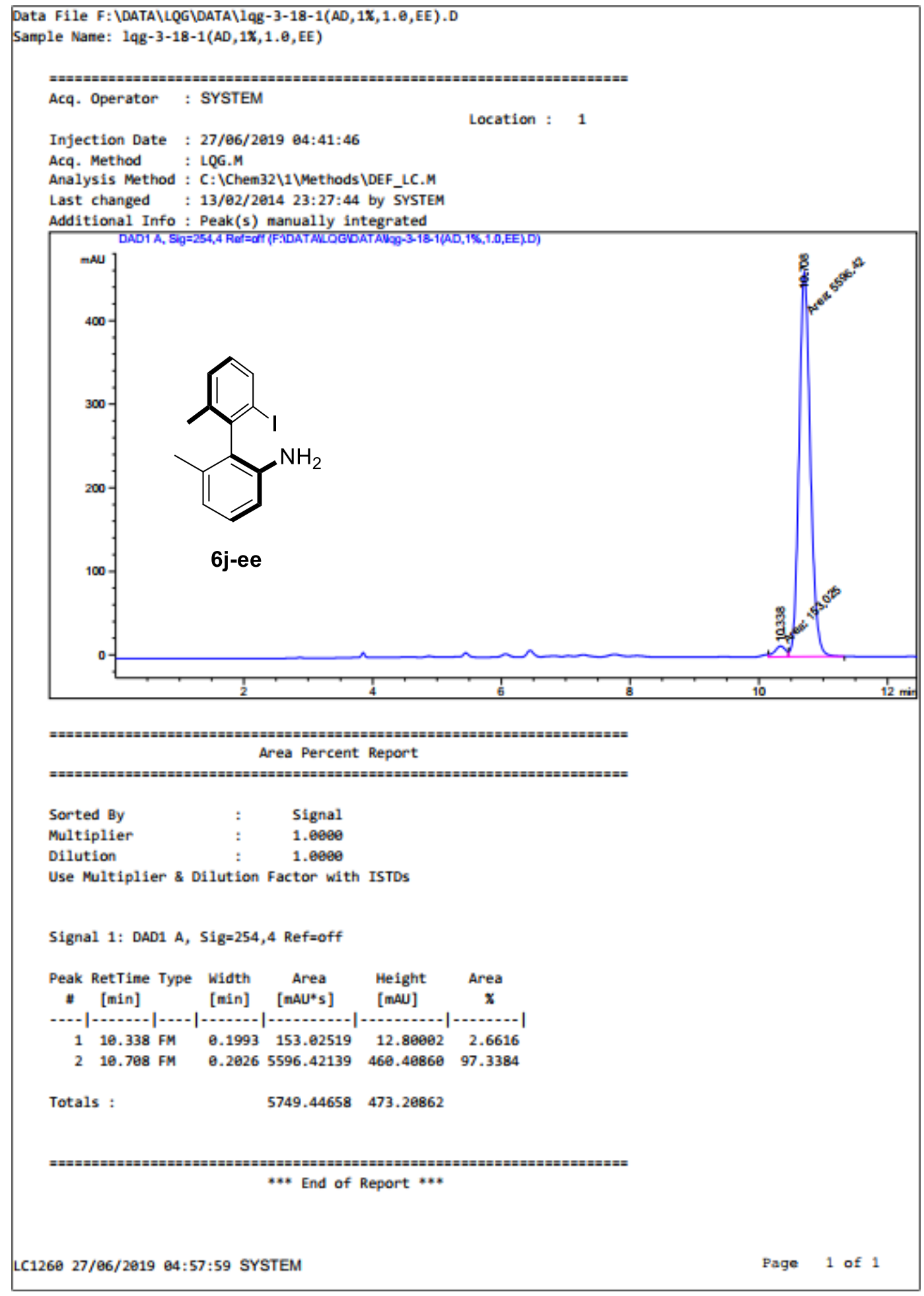




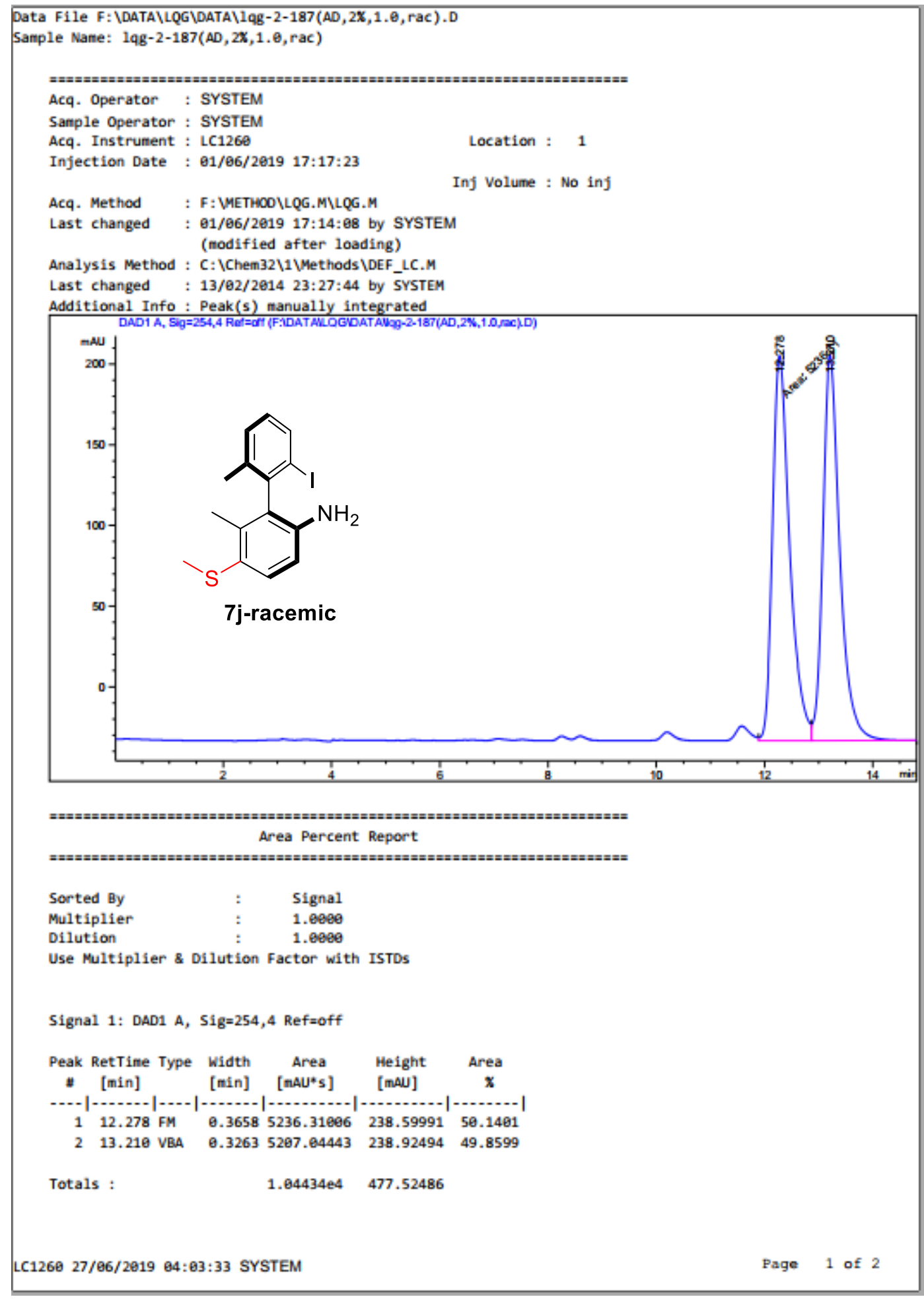




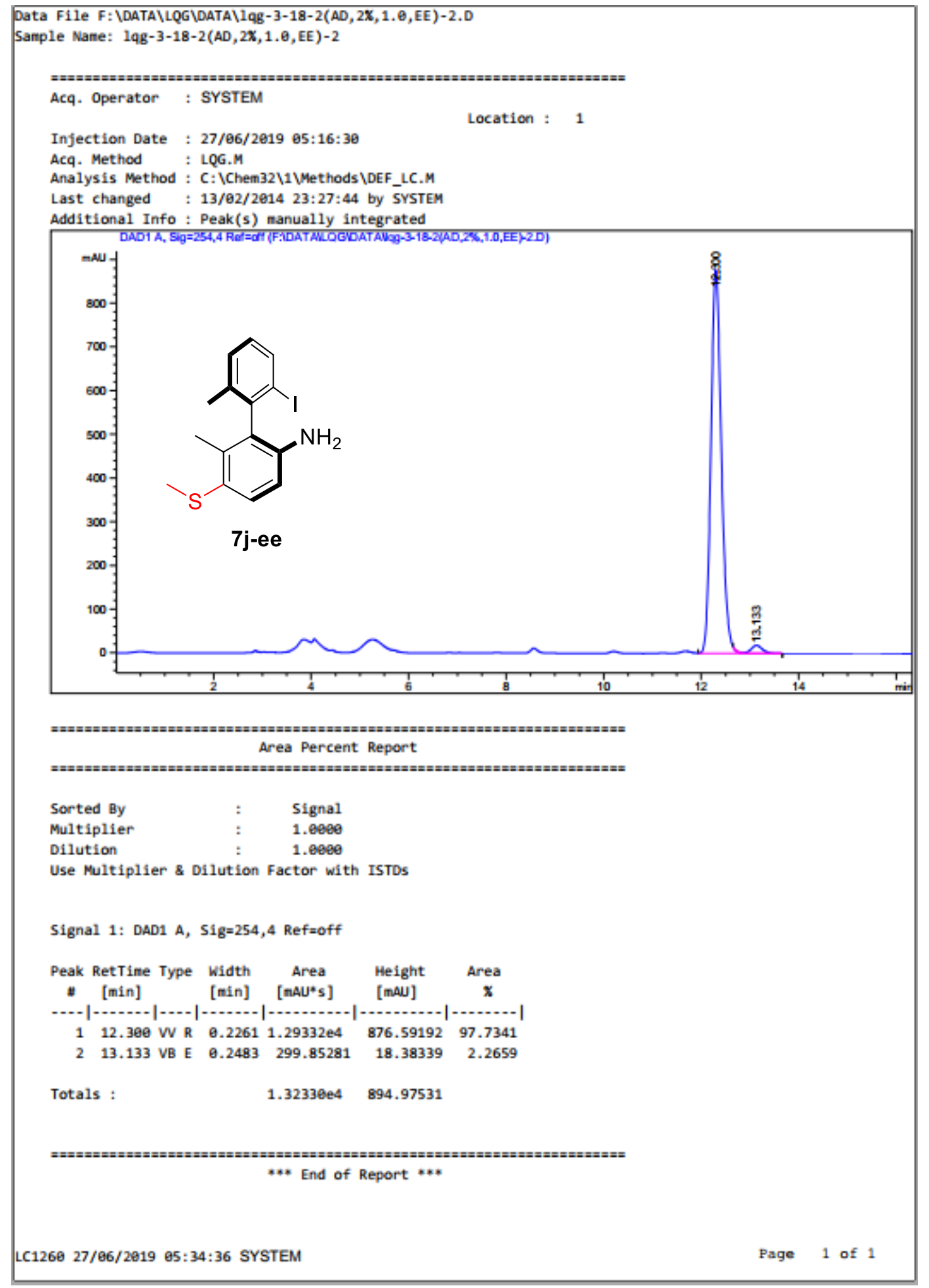

